\title{
Ultrafast Lorentz Microscopy using High-Coherence Electron Pulses
}

\author{
DISSERTATION \\ zur Erlangung des mathematisch-naturwissenschaftlichen Doktorgrades \\ "Doctor rerum naturalium" \\ der Georg-August-Universität Göttingen \\ im Promotionsprogramm / Promotionsstudiengang Physik \\ der Georg-August University School of Science (GAUSS)
}

von

Nara Rubiano da Silva

aus Ribeirão Preto, Brazil

Göttingen, 2019 
Betreuungsausschuss

Prof. Dr. Claus Ropers, IV. Physikalisches Institut

Prof. Dr. Stefan Mathias, I. Physikalisches Institut

Mitglieder der Prüfungskomission

Referent: Prof. Dr. Claus Ropers, IV. Physikalisches Institut

Korreferent: Prof. Dr. Stefan Mathias, I. Physikalisches Institut

Weitere Mitglieder der Prüfungskomission

Prof. Dr. Andreas Tilgner, Institut für Geophysik

Prof. Dr. Ariane Frey, II. Physikalisches Institut

Prof. Dr. Ansgar Reiners, Institut für Astrophysik

Prof. Dr. Alexander Egner, Laser-Laboratorium Göttingen

Tag der mündlichen Prüfung: 29.03.2019 


\section{Abstract}

Information processing and storage applications demand increasing miniaturization and operation speeds, where optical manipulation of magnetic order may play a very important role. It is hence essential to study how light stimulates magnetization reordering at very short spatial and temporal scales. The development of Ultrafast Transmission Electron Microscopy (UTEM), a laser-pump/electron-probe setup, provides a unique means for that. However, magnetic information can only be visualized in UTEM provided a spatially coherent pulsed electron source is available, since phase-contrast techniques such as Lorentz microscopy are required. In this work, highly coherent electron pulses are achieved by implementing a laser-driven Schottky field emitter in UTEM. We employ the electron pulses to map the ultrafast demagnetization of a single magnetic structure with very high spatial and temporal resolution. We show that the transient magnetization can be quantitatively tracked from the image contrast, and that the magnetic order remains rigid with decreased strength on short timescales following the optical excitation. This work builds a solid framework for the acquisition, and quantitative evaluation and interpretation of magnetic contrast images of transient magnetic states. Moreover, it establishes magnetic imaging with high spatiotemporal resolution, and constitutes a step forward in ultrafast phase-contrast imaging on the nanoscale. 



\section{Contents}

1 Introduction 1

2 Methods and Concepts $\quad 5$

2.1 Optical Excitation of Magnetic Materials . . . . . . . . . . . . . 5

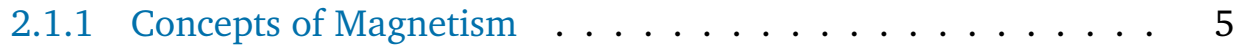

2.1.2 Laser-induced Modification of Magnetic Order . . . . . . . . 8

2.1 .3 Transient Effects . . . . . . . . . . . . . . . . 9

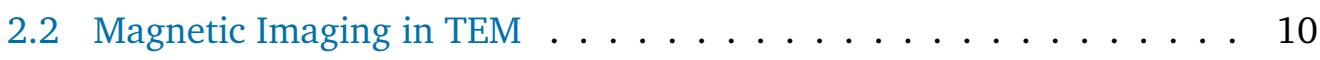

2.2.1 General Description of TEM . . . . . . . . . . . . . 11

2.2.2 Wave-optical Theory of Image Formation . . . . . . . . . . . 12

2.2.3 Techniques for Magnetic Imaging . . . . . . . . . . . 16

2.2.4 Contrast in Fresnel-mode Images ． . . . . . . . . . . . . . 19

2.2 .5 Elements of Holography . . . . . . . . . . . . . . . 22

2.3 Electron Beam Properties for Phase-contrast Imaging . . . . . . . . 23

2.3 .1 Phase Space . . . . . . . . . . . . . . . . . . . 24

2.3.2 Emittance and Brightness . . . . . . . . . . . . 24

2.3 .3 Coherence ..................... . . 26

2.4 Ultrafast Transmission Electron Microscopy . . . . . . . . . . . . 28

2.4.1 Previous Approaches for Magnetic Imaging . . . . . . . . . 29

2.4 .2 The Göttingen UTEM . . . . . . . . . . . . . . . . . . 30

2.4.3 Coherence of Ultrashort Electron Pulses . . . . . . . . . . . 34

3 Aspects and Challenges of fs-Lorentz Microscopy 37

3.1 Sample Design for fs-Lorentz Microscopy . . . . . . . . . . . . . . . 37

3.1 .1 Preparation Details . . . . . . . . . . . . . . . 38

3.1.2 Accumulated Heat Effects on Lorentz Image Contrast . . . . . 40

3.2 Electron Pulse Properties for fs-Lorentz Microscopy . . . . . . . . . . 44 
3.2.1 Performance of Photoemitted Electron Beam for fs-Lorentz Microscopy . . . . . . . . . . . . . . . . 44

3.2.2 Electron Pulses Characterization . . . . . . . . . . . 48

3.3 Data Acquisition and Evaluation . . . . . . . . . . . . . . . 51

3.3.1 Increasing Signal-to-Noise Ratio . . . . . . . . . . . . . . . 52

3.3.2 Drift-Correction Schemes . . . . . . . . . . . 53

4 Numerical Analysis of fs-Lorentz Microscopy 57

4.1 Implementation of Lorentz Image Simulation . . . . . . . . . . . 58

4.1 .1 Electron-sample Interactions . . . . . . . . . . . . . . . 58

4.1.2 Contrast Transfer Function and Image Formation . . . . . . 60

4.1.3 Characterization of Electron-optical Parameters . . . . . . . 61

4.1.4 Characterization of Sample Parameters . . . . . . . . . . . . . 64

4.1.5 Accounting for Limited-coherence Sources . . . . . . . . . . 66

4.2 Results and Discussion . . . . . . . . . . . . . . 67

4.2.1 The Impact of Spatial Coherence . . . . . . . . . . . . 67

4.2.2 Resolution and Contrast in fs-Lorentz Microscopy . . . . . . . 69

4.2.3 Calibration of Transient Magnetization . . . . . . . . . . . 71

5 Ultrafast Demagnetization Imaged by fs-Lorentz Microscopy 75

5.1 Ultrafast Demagnetization of a Magnetic Disc . . . . . . . . . . . . 75

5.1.1 Specific Experimental Details . . . . . . . . . . . . 75

5.1 .2 Image Evaluation . . . . . . . . . . . . . 76

5.1.3 Temporal Evolution of Magnetization . . . . . . . . . . . 79

5.2 Varying Pump-Probe Experiment Parameters . . . . . . . . . . . . . 81

5.2.1 Influence on Time Traces . . . . . . . . . . . . . . . . . 82

5.2.2 Temporal Duration of Electron Probe Pulse . . . . . . . . . . 83

5.2.3 Temporal Duration of Laser Pump Pulse . . . . . . . . . . . 84

6 Conclusion $\quad 89$

6.1 summary . . . . . . . . . . . . . . . . . . . . 89

6.2 Discussion and Outlook . . . . . . . . . . . . . 90

$\begin{array}{lr}\text { A Samples Crystalline Structure } & 97\end{array}$

$\begin{array}{ll}\text { B Simulations Details } & 101\end{array}$ 
Bibliography

Publications and Conferences

Acknowledgements 



\section{Introduction}

The philosophical quest to comprehend all the objects around us, independently of their scale, has always had an ally in visual perception. This stimulated the development of imaging devices such as microscopes and telescopes, which allow us to scrutinize very small or very far objects beyond the human vision capabilities.

In a world eager for miniaturization of electronic devices, microscopy is hugely relevant. Future technological progress demands applications of magnetism, a phenomenon of quantum origin but consequence up to the macroscopic scale. On the one hand, magnetic nanostructures and nanometric spin textures may play the dual role of data storage and logical operation in novel concepts of devices. On the other hand, control of these processes requires an understanding of the mechanisms behind them, demanding investigation tools able to address the dynamical properties of nanometric magnetic elements on ultrashort timescales typically down to femtoseconds.

Spatial information on magnetization dynamics is obtained by a few techniques using optical [1-10] or electron probes [11-18]. The optical methods can easily reach ultrafast temporal resolution, and an associated high resolving power is obtained by employing short-wavelength x-ray radiation, being mostly limited to large scale facilities (synchrotrons and free-electron lasers). Electron microscopes, in comparison, are lab-scale instruments that routinely allow for real-space magnetic imaging with nanometer resolution [19-22]. Achieving a high spatiotemporal resolution, however, can be rather challenging.

Resolutions from the nanosecond down to few hundreds of femtoseconds have been achieved in Transmission Electron Microscopy (TEM) by utilizing pulsed electron beams. Such pulses are obtained in different ways, such as chopping a continuous electron beam [11, 12], or creating electron bunches containing few [23-28] to several thousand electrons $[29,30]$ each. Still, squeezing several electrons in a short electron pulse may degrade the beam coherence and thus restrain the practicable 
spatial resolution. Since contrast from magnetic samples is obtained in TEM using phase-contrast techniques such as Lorentz microscopy, low coherence constitutes a major impediment.

Therefore, in parallel to the historical development of static TEM, the capabilities of time-resolved TEM in general, and of magnetic imaging in particular, rely on the development of high-coherence pulsed electron sources. Previous realizations of magnetic imaging in time-resolved TEM $[16,18]$ have employed photoemission from thermionic electron guns, yet much improvement can be realized by using field emission guns. We have recently shown that, by employing a laser-driven, tip-like nanocathode [31-33] in a field emission gun, record electron pulse properties are obtained, especially regarding spatial coherence [28], conditions particularly suited for magnetic imaging with high spatiotemporal resolution.

In this thesis, we demonstrate the capabilities of our approach to Ultrafast Transmission Electron Microscopy (UTEM) for real-space magnetic imaging with a previously unachieved combination of spatial and temporal resolution. In particular, defocused micrographs of optically excited magnetic nanostructures are obtained with two purposes in mind: firstly, for evidencing the quality of our electron source design for magnetic imaging, and secondly, for establishing the capability of time-resolved TEM for mapping transient magnetic fields on the nanoscale with high temporal resolution. Our findings constitute an important step towards the establishment of phase-contrast techniques in ultrafast TEM, and a valuable addition to the available tools for imaging out-of-equilibrium systems.

\section{Outline}

This thesis is structured as follows. Chapter 2 introduces relevant conceptual and methodological aspects of magnetism, electron microscopy of magnetic materials (Lorentz microscopy) and Ultrafast Transmission Electron Microscopy. In Chapter 3, we present technical aspects for the realization of ultrafast Lorentz microscopy. Schemes to overcome the challenges associated with undesirable laser heating of the sample during the experiments and with low dose illumination are established. Next, Chapter 4 presents a numerical analysis of the capabilities of ultrafast Lorentz microscopy. Moreover, it demonstrates the potential to quantitatively analyze the 
image contrast in terms of the transient sample magnetization. In Chapter 5, we apply ultrafast Lorentz microscopy to map the temporal evolution of magnetic systems undergoing optically-induced magnetization dynamics. We analyze the observed demagnetization dynamics of a single magnetic island, and demonstrate the achievable spatiotemporal resolution of the technique. The final part, Chapter 6, summarizes the work done within this thesis, presents a discussion comparing our technique to other time-resolved methods for magnetic imaging, and an outlook of possible sample systems to be investigated and future developments. 



\section{Methods and Concepts}

THIS CHAPTER PRESENTS the basic theoretical and experimental background for the development of this work. First, a brief introduction to relevant concepts of excitation of magnetic materials using light is given (Section 2.1). Next, the imaging techniques in Transmission Electron Microscopy that can be applied for magnetic materials are revised, along with a theoretical description of image formation (Section 2.2). We then introduce the essential electron beam properties for employing these imaging methods (Section 2.3). In the end, we describe the development of the main experimental platform used in this thesis: Ultrafast Transmission Electron Microscopy (Section 2.4).

\subsection{Optical Excitation of Magnetic Materials}

The usual way to manipulate magnetic order in data storage technologies is by using magnetic fields. After the first demonstration of sub-ps demagnetization by 60 -fs laser pulses [34], however, there is an ever increasing interest in optical excitation of magnetic materials, pushed by the appealing idea of using light for controlling magnetization with higher speeds.

In this section, an overview of relevant concepts for understanding magnetic order and its dynamical behaviour is given first. Next, selected effects of optical excitation of magnetic materials, both in equilibrium and transient, are presented. In general, we follow Ref. [35, 36], and references for specific parts are given.

\subsubsection{Concepts of Magnetism}

Micromagnetism is a continuum theory of magnetically ordered materials, which successfully describes the classical magnetization field $\vec{M}$ at the microscopic scale [35]. The theory provides adequate treatment of the magnetic phenonema within this thesis, namely the formation of magnetic domains and domain walls in confined structures, both in equilibrium and out-of-equilibrium. Here, we present the fun- 
damental concepts only qualitatively. Details are given in Ref. [35] and references therein.

Within this theory, the magnetic order is described by the density of magnetic moments, often given by the reduced field $\vec{m}(\vec{r})=\vec{M}(\vec{r}) / M_{s}$, where $M_{s}$ is the saturation magnetization. This vector quantity is determined based on the variational principle, so that the total free energy is minimized under the constraint $|\vec{m}|^{2}=1$ [35]. Notably, there are local and non-local contributions to the free energy. The most important energy contributions according to their usual order of magnitude are:

1. Exchange energy (local): of quantum-mechanical origin; it is minimized when nearest neighbor spins are aligned (either parallel or antiparallel).

2. Anisotropy energy (local): resulting from spin-orbit interactions; it is minimized when $\vec{m}$ is ordered according to the structural axes of the material (both for the undisturbed crystal structure and for deviations from it, for instance, because of lattice defects).

3. Zeeman energy (local): classical energy contribution, which is minimized when $\vec{m}$ is aligned to applied external fields.

4. Stray field energy (non-local): originating from the classical interaction between magnetic dipoles and associated with the field generated by the divergence of the magnetization $\vec{M}$; it is minimized by avoiding the formation of "magnetic charges". As a result, $\vec{M}$ tends to form flux-closure patterns, and the spins tend to align to surfaces and edges of a magnetic system.

By applying the variational principle to the total free energy, one obtains the micromagnetic equations describing the magnetic system. Hence, it is possible to calculate (often numerically) the static magnetic configuration, as well as its dynamic evolution, for example, upon the application of an external magnetic field or variations in the saturation magnetization $M_{s}$ (e.g., by heating or cooling) [35]. With growing computer power, the predictive capabilities of software packages such as mumax3 [37] is increasing in quality and capacity of dealing with larger systems.

In confined magnetic structures, such as the $\mu \mathrm{m}$-sized islands investigated in this thesis, the stray field energy gets more relevant due to the substantial presence 
(a)
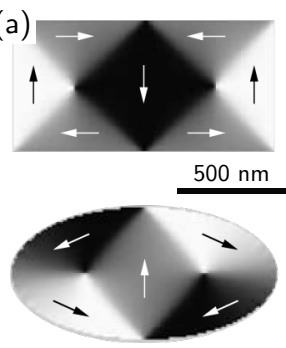

(b)

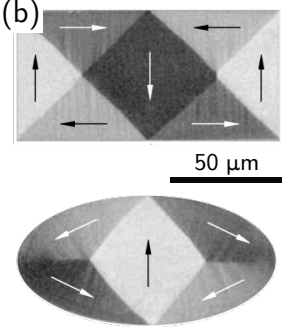

(c)

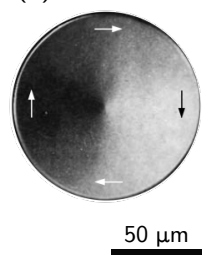

(d)

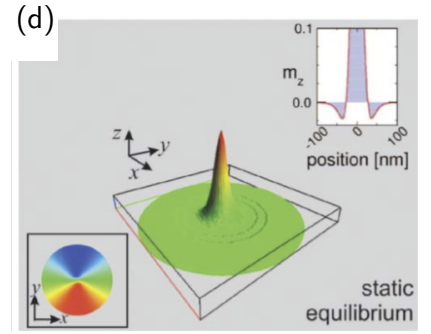

Fig. 2.1.: Examples of magnetic domains formed in confined structures. (a) Calculated domains for 20-nm thick permalloy elements. (b) Magneto-optical mages of 240-nm thick permalloy elements, showing the domain structure calculated for (a). (c) Magneto-optical image of an iron-permalloy multilayered element of 300-nm thickness in a magnetic vortex state. (d) Simulated out-of-plane $\left(m_{z}\right)$ and in-plane ( $m_{x}$, bottom inset) magnetization components in a permalloy disc of 20-nm thickness and 100-nm radius in a vortex state. Red: $m_{x}=1, m_{z}=1$; blue: $m_{x}=-1, m_{z}=-1$. (a-b) Adapted by permission from Springer Nature Customer Service Centre GmbH: Springer [35], (C) Springer-Verlag Berlin Heidelberg (1998). (c) Adapted from Ref. [35], but originally published in Ref. [41]. Reprinted from [41], with the permission of AIP Publishing. (d) Reprinted figure with permission from [42]. Copyright (2007) by the American Physical Society.

of edges, and eventually defines the magnetic domain configuration. Figure 2.1 demonstrates the domain patterns formed in magnetic structures of different shapes. In particular, thin magnetic discs show a curling magnetization pattern (a magnetic vortex) characterized by a homogeneous $|\vec{m}|$ lying in-plane everywhere but very close to the center, where a singularity is formed and $\vec{m}$ points out of plane $[35,38$, 39]. The curling pattern is the ground state also in elements of different shapes, whose dimensions are too small for the formation of domain walls to be energetically favorable, and too large to form a single domain [40].

Various mechanisms and processes associated with magnetization dynamics in nanostructures are shown in Figure 2.2, sorted according to their corresponding time and length scales. The processes related to optical excitation are included; it is commonly agreed that optical excitation of magnetic material results in thermal processes (associated with out-of-equilibrium electrons, spins and phonons) and coherent processes (associated with orbital and spin angular momenta).

Detailed description of the mechanisms and processes are given in Ref. $[36,43]$ and references therein. Here, we present only selected effects in the following sections. 


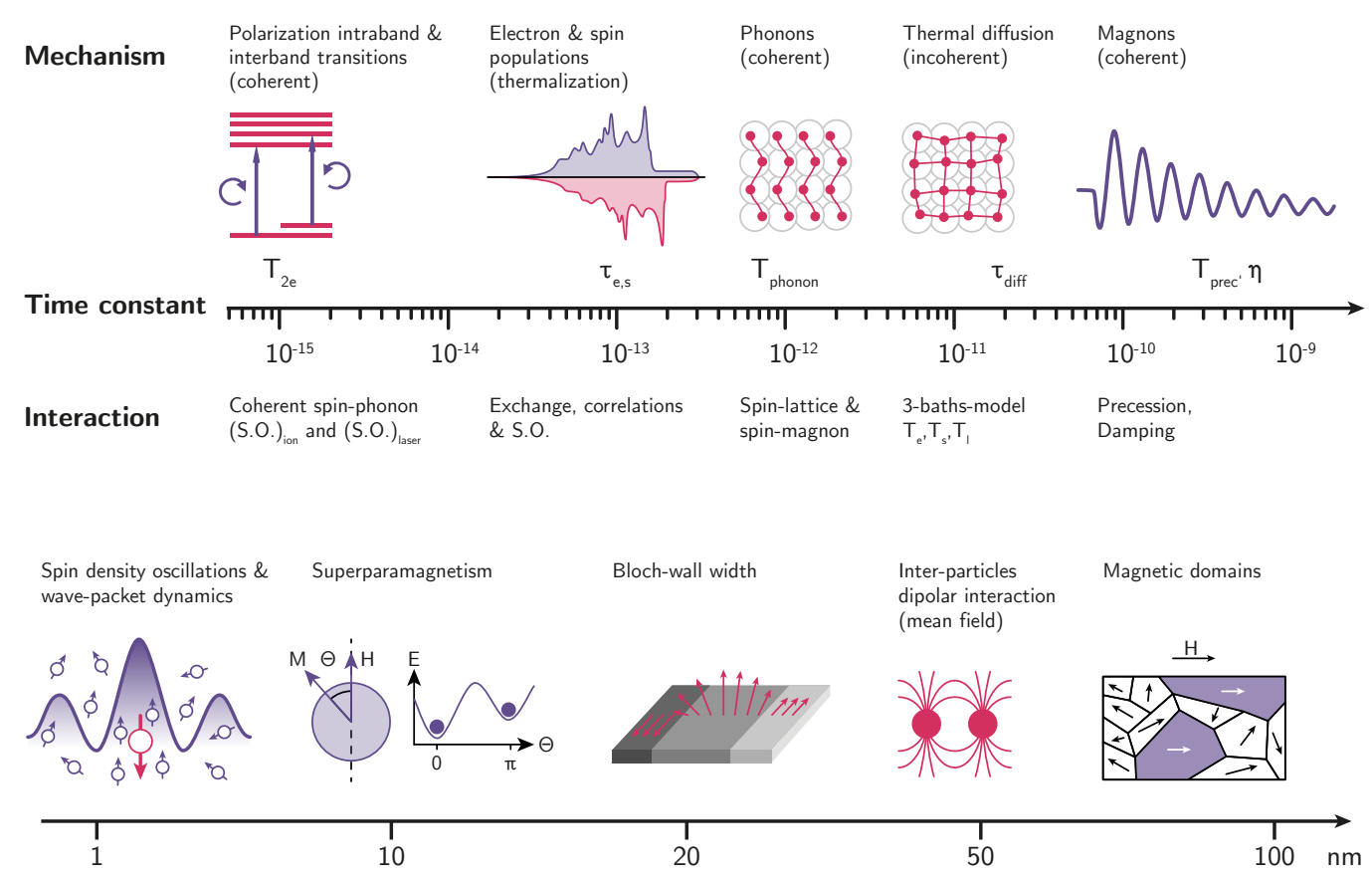

Fig. 2.2.: Temporal (top panel) and spatial (bottom panel) scales in magnetism. Redrawn from Ref. [43].

\subsubsection{Laser-induced Modification of Magnetic Order}

We now illustrate long-lived modifications in magnetic order, excited by ultrashort laser pulses. Optical manipulation of magnetic materials is presented in Figure 2.3a. Magnetization is deterministically switched (i.e., reversed) by illumination with linearly or circularly polarized laser pulses. This effect has been found for specific magnetic materials or material classes [44-52].

Figure 2.3b displays a laser-induced magnetic texture. The initial magnetic structure is strongly driven to an out-of-equilibrium state by the absorption of the laser pulse energy and cooling down to the initial temperature after undergoing a transition to a different magnetic state. Specifically, for fast cooling rates, topologically protected structures may remain, such as the vortex-antivortex networks we previously observed in continuous films [53]. More recently, the same phenomena was investigated in islands [54].

The effects presented here are merely illustrative of the richness of laser-induced effects on magnetic materials. The next section deals with the main processes addressed by our experiments in Chapter 5 . 


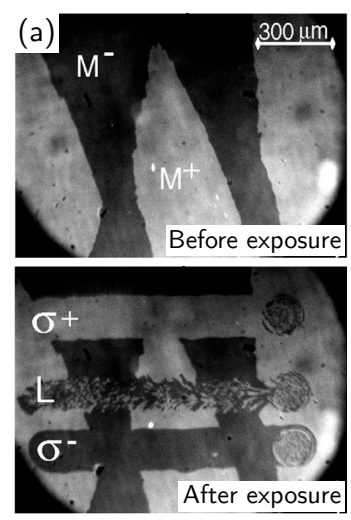

(b)

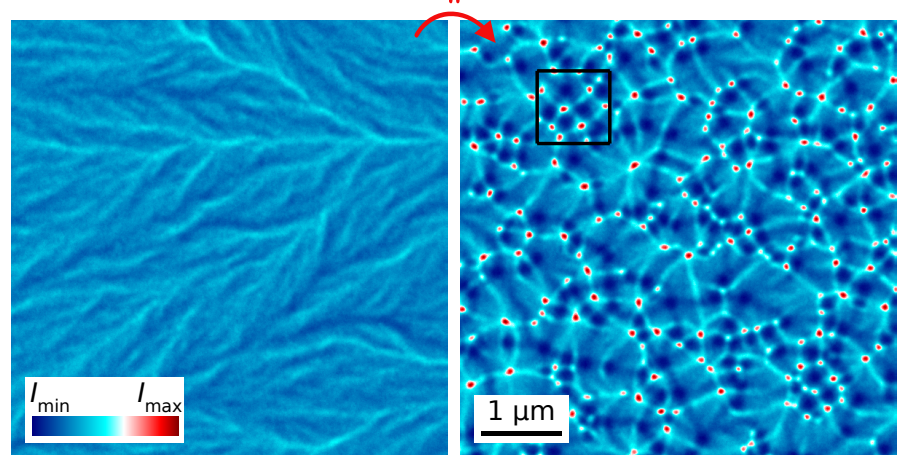

Fig. 2.3.: Examples of manipulation of magnetic order by laser illumination. (a) Magnetooptical images of $\mathrm{Gd}_{22} \mathrm{Fe}_{74.6} \mathrm{Co}_{3.4}$ films before and after sweeping ultrashort polarized laser pulses. The change in contrast evidence magnetic domain switching. (b) Transmission electron micrographs reveal that after a single, intense femtosecond laser pulse a metastable network of vortices and antivortices is formed in a continuous iron thin film. (a) Reprinted figure with permission from [44]. Copyright (2007) by the American Physical Society. (b) Reprinted figure with permission from [53]. Copyright (2017) by the American Physical Society.

\subsubsection{Transient Effects}

In 1996, an unexpected effect of femtosecond laser pulses on magnetic materials was observed for the first time: the ultrafast demagnetization following excitation [34]. This work initiated the research on femtomagnetism; inspiring the discovery of further intriguing phenomena such as the ones mentioned in the previous section.

The archetypal ultrafast demagnetization curve is shown in Figure 2.4a, and evidences that the spins are involved in processes occurring faster than typical electronlattice equilibration time scales in metals [55-57]. A phenomenological picture that qualitatively describes the laser-induced demagnetization is the three-temperature model (3TM) [34], depicted in Figure 2.4c. The model considers individually thermalized electron, spin, and lattice reservoirs, coupled to each other by interactions of various origins and efficiencies. When the optical energy is absorbed by the electrons, a rapid electron-spin coupling (timescale of few $100 \mathrm{fs}$ ) leads to an ultrafast drop of the magnetization. Subsequently, coupling with the lattice brings the three reservoirs to thermal equilibrium in timescales on the order of 1 ps.

The underlying mechanisms of ultrafast demagnetization are current fields of research, and there is still active debate on their relative importance in different 

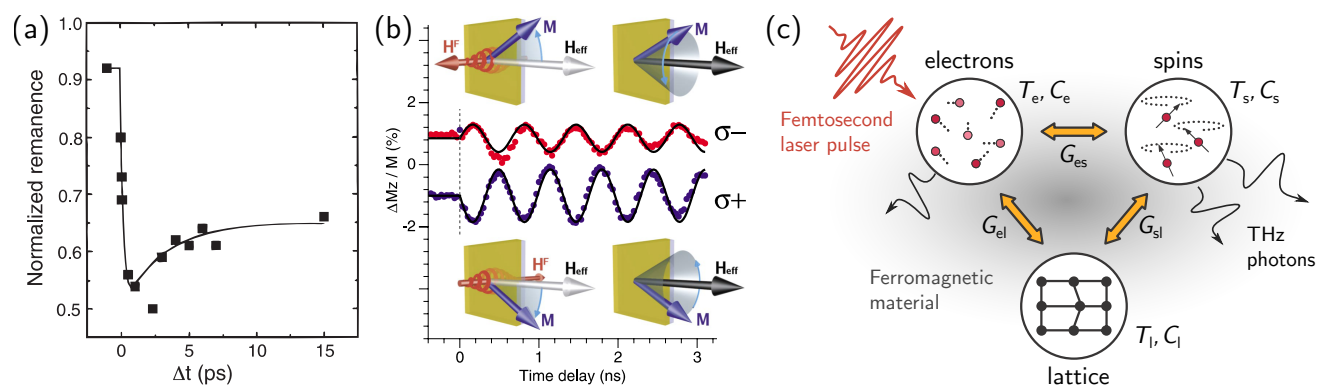

Fig. 2.4.: Transient laser-induced effects on magnetic materials. (a) Ultrafast demagnetization of a Ni film following excitation by a 60 -fs laser pulse revealed by pump-probe spectroscopic measurements. (b) Optically induced magnetization precession in garnet films observed using an all-optical pump-probe spectroscopic technique. The phase and amplitude of the precession is determined by the circular polarized light handedness. (c) Schematic representation of the three-temperature model, depicting the reservoirs and the energy transfer channels between them. Terahertz photons are emitted during the demagnetization. (a) Reprinted figure with permission from [34]. Copyright (1996) by the American Physical Society. (b) Reprinted figure with permission from [58]. Copyright (2005) by the American Physical Society. (c) Redrawn from Ref. [59].

magnetic systems. Possible mechanisms are direct spin-photon interactions [59], optically driven superdiffusive spin transport [60], and spin-flip scattering [61].

Laser excitation can also trigger coherent transient processes. One example is the coherent precession following excitation with circularly polarized light (Figure 2.4b). More examples of laser-induced effects are given in Ref. $[36,43]$ and references therein.

The majority of the investigations on transient magnetization were performed using ultrafast optical spectroscopy techniques. When studying the same effects in confined magnetic structures, however, spatial information is required, and imaging techniques allowing for the observation of the transient magnetization distributions are essential [43]. Considering the dimensions of relevant magnetic phenomena (see Figure 2.2), spatial resolution in the range of $10 \mathrm{~nm}$ is desirable. The development of electron microscopy with a combined spatiotemporal resolution suitable for visualizing the transient structures will be presented in this thesis.

\subsection{Magnetic Imaging in TEM}

In order to assess the laser-induced changes in nanostructured magnetic materials, we employ Transmission Electron Microscopy (TEM). In this section, a brief 
description of the instrument and its application to magnetic imaging is given. In particular, we explain the image formation in TEM, invoking essential concepts for the emergence of TEM contrast from magnetic samples, and for the development of the image simulations performed in Chapter 4. The technical details on the instrumentation will be introduced in Section 2.4.2.

\subsubsection{General Description of TEM}

TEM is a versatile tool offering one of the highest resolutions currently achievable for probing condensed matter [62-64]. Making use of highly energetic electrons (in the range of 20-3000 keV), the instrument provides the means for recording atomically resolved real-space images, diffraction patterns and energy spectra of nanometerscale regions. In other words, the real and reciprocal spaces, and spectroscopic and composition information of a bulk material can be assessed in a single instrument.

The highly energetic electrons employed in TEM interact with the sample under investigation in several ways, as depicted in Figure 2.5 [62-64]. Here, specifically, we are interested in the interaction of the imaging electrons with the magnetic field created by the sample. Classically, the interaction is represented as a deflection in the electron trajectory by the action of the Lorentz force. Quantum-mechanically, the interaction is described as a linear phase added to the electron wave function, and the phase shift imparted to electrons travelling through a region with an electrostatic potential $V$ and a magnetic vector potential $\vec{A}$ is given by the Aharonov-Bohm (AB) phase shift [65]:

$$
\phi(\vec{r})=\frac{e}{\hbar}\left(\frac{1}{v} \int V(\vec{r}, z) d s-\int \vec{A}(\vec{r}, z) \cdot \mathrm{d} \vec{s}\right),
$$

where $e$ is the elementary electronic charge, $\hbar$ is the reduced Planck constant, $v$ the electron velocity, $\vec{r}$ the spatial vector perpendicular to the electron trajectory, and the integrals are computed along the electron propagation direction $z$.

Since the primary information about magnetic materials is encoded in the phase of the electrons transmitted through the sample $[66,67]$, we must use phase contrast techniques, which can only be understood in the wave picture of image formation presented in the next section. 


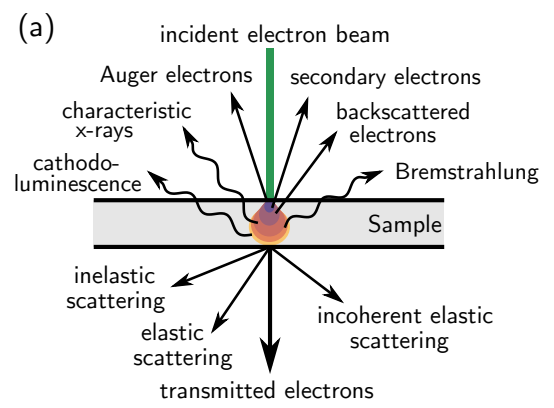

(b)

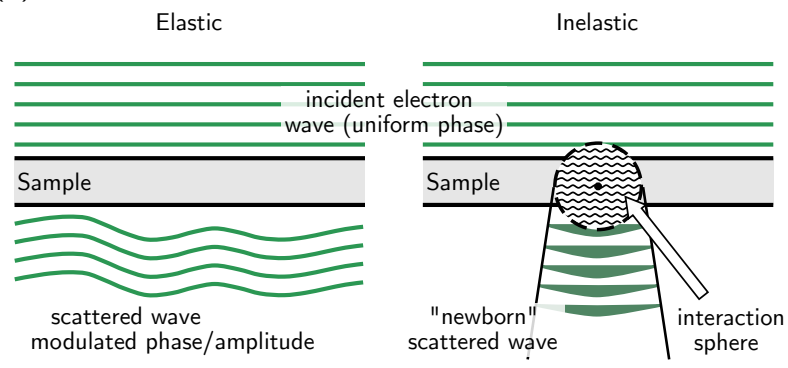

Fig. 2.5.: Electron-sample interactions in TEM. (a) The electrons interact with a sample in a multitude of processes, generating signals of electronic (straight arrows) and electromagnetic (curly arrows) nature. The interaction volume (pear-shaped region) becomes smaller for higher incident electron energies and thinner specimens. (b) Elastic and inelastic interactions of the electron wave (ideally a plane wave) with the specimen in TEM. Panel (b) was redrawn from Ref. [68].

\subsubsection{Wave-optical Theory of Image Formation}

This section covers the image formation in TEM within the wave-optical picture. Some concepts are carefully elaborated, given their relevance for the image simulations in Chapter 4. We present first a qualitative representation based on a ray diagram, and then follow the microscope transfer function formalism. This section is based on the comprehensive formulations given in Ref. [62, 69].

The image formation in TEM is illustrated in Figure 2.6a, which shows only its basic elements disregarding the correct number and position of the lenses. For simplicity, we show here a ray diagram, but the wave nature of the electrons is incorporated at the right of Figure 2.6a, where the corresponding electron wave function at each stage is given. Initially, a spherical wave is emitted from the electron source, being subsequently collimated by the condenser lens system to form a plane wave impinging on the sample. After being modified by interactions with the sample, the electron exit wave function $\psi(\vec{r})$ is collected by the objective lens, whose back focal plane (BFP) is located a distance $f$ after the lens. The net effect of propagation through the objective lens system (objective lens and apertures) can be written using the so called contrast transfer function $T(\vec{q})$, defined at the BFP, which is equal to unity for a perfect lens. Therefore, at the BFP, the wave is described by the Fourier transform of $\psi(\vec{r})$ modulated by $T(\vec{q})$. The projection system further magnifies the image $\mathcal{F}^{-1}[T \mathcal{F}(\psi)]$ formed at the image plane of the objective lens. The detected signal is the squared amplitude of the final electron wave function. In contrast to 


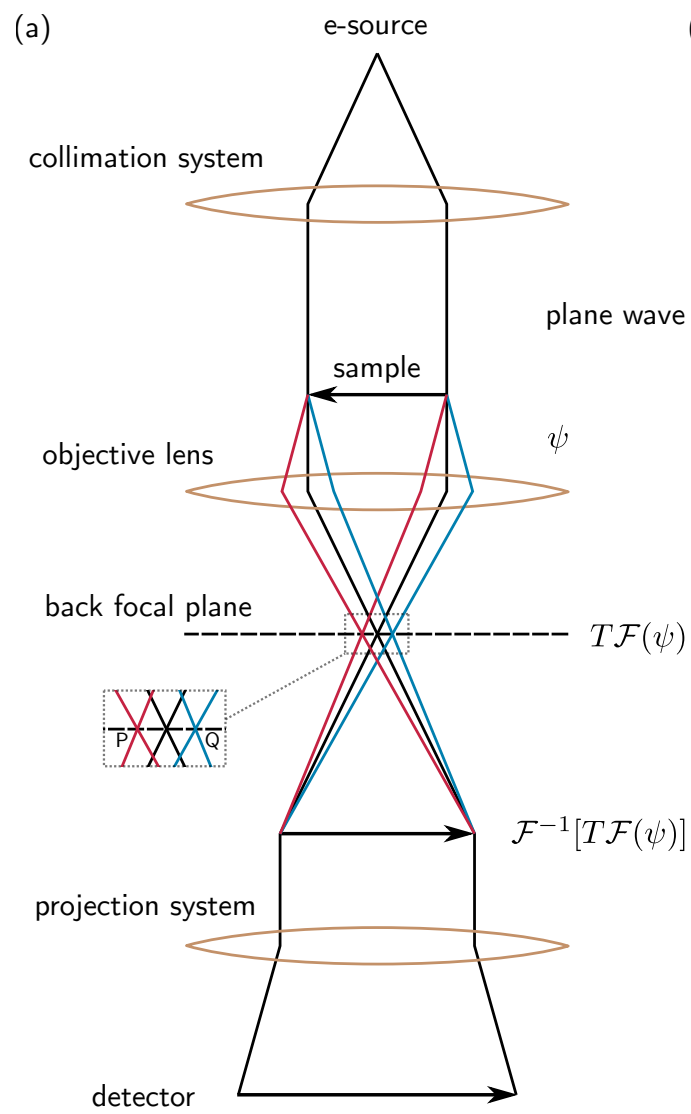

(b)

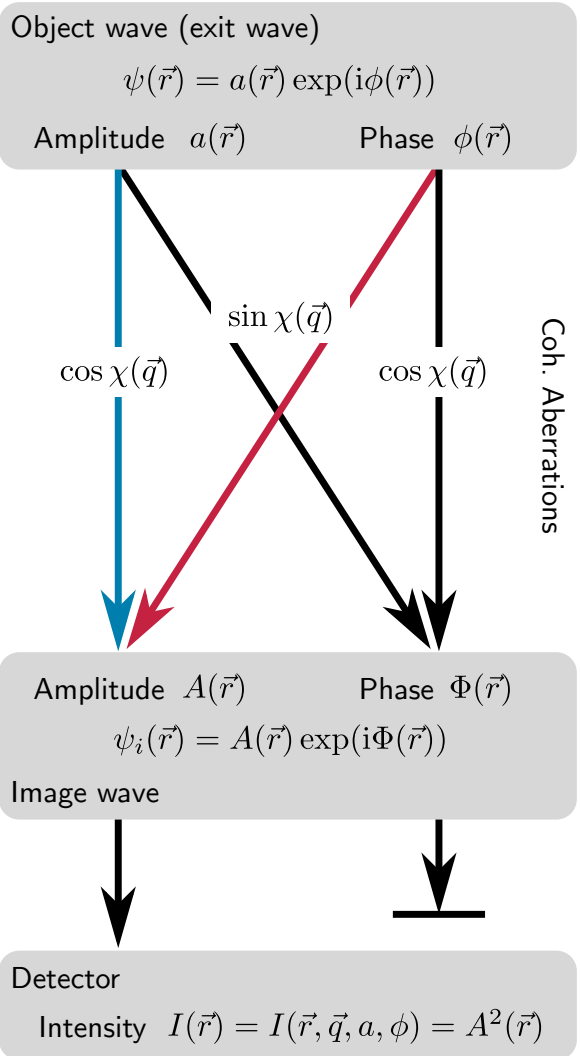

Fig. 2.6.: Image formation in TEM. (a) Schematic description of wave propagation, interaction with sample and image formation. (b) Information transfer channels along the imaging system in TEM, including coherent aberrations. Only the squared amplitude of the image wave is recorded, but it contains information on the phase of the object wave function. Panel (b) was redrawn from Ref. [70].

the objective lens, aberrations introduced by the projection system lenses can be neglected.

The image formation can also be understood from the perspective of an information transfer channel $[62,70]$, as depicted in Figure 2.6b. Due to the coherent aberrations described by a phase distribution $\chi(\vec{q})$, there are four transfer channels from the exit wave to the image wave, resulting in a complex mixing of amplitude and phase information, which is transferred to the detected signal only via the image wave amplitude. Note that a considerable amount of information is lost at the phase channel. The image formed in TEM can hence be computed from the exit wave function $\psi(\vec{r})$ using:

$$
I=\left|\mathcal{F}^{-1}[T \mathcal{F}(\psi)]\right|^{2} .
$$


The transfer function $T$ collects all properties of the main imaging system of the microscope, i.e. the objective lens, for given illumination conditions. Specifically, $T$ includes coherent and incoherent aberrations. The former is associated with the objective lens and mixes up amplitude and phase of the exit wave function $\psi$ (see Figure 2.6b). The later relates to the limited degree of coherence of the illumination and damps information transfer. The effects of both aberrations can be understood by considering how the wave is reordered at the BFP. As shown by the black and red ray diagrams in Figure 2.6a, the undeflected part of the wave is focused at the optical axis, whereas parts deflected by the same angle are focused at a lateral distance $q$ from it. Looking at a single point in this plane (for instance, the point $\mathrm{P}$ ), we see that the wave there contains information from different parts of the sample, i.e., with different amplitude and phase. The resulting interference scrambles phase and amplitude information in what is called coherent aberrations [70]. In addition, the extent to which this mixture can occur is limited by the phase variations of the illumination wave at different parts of the sample due to fluctuations in wavelength (temporal coherence) or in incidence angle (transverse spatial coherence). Such phase variations therefore damp the information that can be transferred by the imaging system, in what is called incoherent aberrations [70].

In general, we can write the contrast transfer function $T$ as follows [62]:

$$
T(\vec{q})=E^{t c}(\vec{q}) E^{s c}(\vec{q}) A_{B F P}\left(\vec{q}-\vec{q}_{0}\right) \mathrm{e}^{-\mathrm{i} \chi(\vec{q})},
$$

where the function $\chi$ represents the coherent aberrations, while $E^{t c}$ and $E^{s c}$ denote the temporal and spatial coherence damping envelope functions, respectively. The function $A_{B F P}$ represents any aperture positioned around the point $\vec{q}_{0}$ at the BFP.

The coherent aberration function $\chi$ can be written as $[62,71]$ :

$$
\begin{aligned}
\frac{\chi(\vec{q})}{2 \pi}= & -\frac{1}{2} \lambda q^{2}\left[\Delta f+C_{a} \cos 2\left(\phi-\phi_{a}\right)\right] \\
& -\frac{1}{3} \lambda^{2} q^{3}\left[G \cos 3\left(\phi-\phi_{G}\right)+3 K \cos \left(\phi-\phi_{K}\right)\right] \\
& -\frac{1}{4} \lambda^{3} q^{4}\left[H \cos 4\left(\phi-\phi_{H}\right)-C_{s}\right] \\
& + \text { higher order terms, }
\end{aligned}
$$


where $\Delta f$ is the defocus (distance between actual final image plane and detector plane), $C_{s}$ is the objective lens spherical aberration, and the pair $\left(K, \phi_{K}\right)$ is the axial coma $K$ and the corresponding reference angle at the BFP. The pairs $\left(C_{a}, \phi_{a}\right)$, $\left(G, \phi_{G}\right)$, and $\left(H, \phi_{H}\right)$ represent the two-fold, three-fold and four-fold astigmatism, respectively, in an analogous manner.

The incoherent aberrations can be taken into account as follows. The intrinsic spread of electron kinetic energies relates to a spread of the possible wavenumbers $k=1 / \lambda$ contributing to the electron beam (temporal coherence). The focal length will thus vary for different electron energies and the resulting image is an incoherent summation of image intensities from a distribution of focal lengths. This contribution is given by the temporal coherence envelope [70]:

$$
E^{t c}(\vec{q})=\exp \left[-\frac{\pi^{2}}{2}\left(\lambda C_{c} \sqrt{\frac{\sigma_{E}^{2}}{\left(e U_{a}^{*}\right)^{2}}}\right)^{2} q^{4}\right]
$$

where $C_{c}$ is the chromatic aberration constant, and $\sigma_{E}^{2}$ is the standard deviation of the electron energy distribution centered at the nominal energy $e U_{a}^{*}$. The relativistically modified acceleration potential $U_{a}^{*}$ is given by $U_{a}\left(1+\frac{e U_{a}}{2 m_{o} c^{2}}\right)$, in which $U_{a}$ is the experimental acceleration voltage, $m_{0}$ the electron rest mass and $c$ the speed of light.

We can treat the influence of the illumination angular spread (spatial coherence) on the imaging process in a similar manner. Plane waves with different incidence angles $\vec{\alpha}_{i l l}$, corresponding to spatial frequency $\vec{q}=k \vec{\alpha}_{i l l}$, contribute incoherently to the final image $I(\vec{r})$. We can thus compute $I(\vec{r})$ as the sum of all the images resulting from a single $\vec{\alpha}_{i l l}$, weighted by the normalized distribution $i(\vec{Q})$ of illumination directions of the source [70]:

$$
I(\vec{r})=\int_{\text {source }} i(\vec{Q}) I_{\vec{Q}}(\vec{r}) \mathrm{d} \vec{Q}
$$

For partial coherence, the range of illumination directions which have to be taken into account is related with the angle $\sigma_{\theta}$ subtended by the (virtual) source width at a single point in the sample plane. The angle $\sigma_{\theta}$ corresponds to the transverse coherence length $l_{\perp}$ by:

$$
l_{\perp}=\frac{\hbar}{m_{0} c \beta \gamma} \frac{1}{\sigma_{\theta}} .
$$


It is thus possible to describe the distribution of the illumination directions using a rotationally symmetric Gaussian function with spread given by $\sigma_{\theta}$ :

$$
i(\vec{Q})=\frac{1}{\pi Q_{0}^{2}} \mathrm{e}^{-\left(Q^{2} / Q_{0}^{2}\right)}
$$

where $Q_{0}=k \sigma_{\theta}$.

For small variations in illumination direction, the transfer function for tilted illumination can be approximated by the Taylor expansion $\chi(\vec{q}+\vec{Q}) \approx \chi(\vec{q})+\vec{Q} \nabla \chi(\vec{q})$, and the image intensity is calculated by modifying the transfer function by the spatial coherence envelope [70]:

$$
E^{s c}(\vec{q})=\exp \left[-\pi^{2} \frac{\sigma_{\theta}^{2}}{\lambda^{2} \ln (2)}\left(C_{s}(\lambda q)^{3}+\Delta f(\lambda q)\right)^{2}\right]
$$

We apply Equations 2.3, 2.4, 2.5 and 2.9 within the wave formalism to simulate TEM images of a magnetic nanoisland (see Chapter 4). Next, we present the TEM techniques for imaging magnetic structures.

\subsubsection{Techniques for Magnetic Imaging}

As seen in Section 2.2.1, the magnetic information acquired from a sample modifies the phase of the exit wave. Therefore, it is essential to imprint phase information on the acquired signal. The assortment of techniques for recording magnetic phase information are generally denominated Lorentz microscopy [63].

We can begin to understand the Lorentz microscopy techniques by looking at Equations 2.3 and 2.4. For simplicity, let us assume a perfectly coherent source (i.e., both $E^{s c}$ ad $E^{t c}$ are equal to unity), and an astigmatism-corrected, coma-free microscope, for which the coherent aberration function is given by:

$$
\frac{\chi(\vec{q})}{2 \pi}=-\frac{1}{\lambda}\left[\frac{1}{2} \Delta f(\lambda q)^{2}-\frac{1}{4} C_{s}(\lambda q)^{4}\right]
$$

and therefore

$$
T(\vec{q})=A_{B F P}\left(\vec{q}-\vec{q}_{0}\right) \exp \left[\frac{2 \pi \mathrm{i}}{\lambda}\left(\frac{1}{2} \Delta f(\lambda q)^{2}-\frac{1}{4} C_{s}(\lambda q)^{4}\right)\right]
$$


Recalling that the coherent aberration function $\chi$ effectively mixes the phase and amplitude of the exit wave function, we see from this equation that the most straightforward way to introduce magnetic contrast in the final image is to get out-of-focus images $(\Delta f \neq 0)$, the so-called Fresnel mode of Lorentz microscopy. This is achieved in TEM by strengthening/weakening the objective lens excitation, which modifies its focal length and, as a result, the position of the back-focal plane (BFP) and the image plane along the optical axis of the microscope (the detector plane is fixed, see Figure 2.6). A second way is to filter the wave in the BFP by using an aperture displaced so that only contributions from phase shifts induced by specific magnetization directions (see Equation 2.1) are further propagated. This constitutes the Foucault mode of Lorentz microscopy.

In Figure 2.7, the formation of image contrast from magnetic samples is schematically shown for the main methods. As a reference point, Figure 2.7a shows a usual, infocus TEM image with no magnetic contrast of a two-domain thin film. Note that an incident electron beam (green) acquires a phase shift depending on the direction of the magnetic field produced by the sample, corresponding to a deflection in different directions (blue and red beams). The final in-focus image is formed by electron wave interference in the detector plane in a way that no information about the deflection (phase shift) is recorded. In Figures 2.7b-e, the methods for magnetic imaging listed below are illustrated $[62,66,69,72]$. Figure 2.8 demonstrates the imaging modes for magnetic islands of different shapes.

- Fresnel mode of Lorentz microscopy: the objective lens is defocused, and the BFP is shifted. In the detector plane, the electrons deflected by neighboring domains will interfere at the domains walls, introducing contrast features in the image of this region.

- Foucault mode of Lorentz microscopy: an aperture is inserted at the BFP, selecting only the electrons deflected by specific domains. The electrons going through other domains will not contribute to the image, which will appear darker in the recorded image.

- Differential phase contrast: a focused electron beam is scanned across the sample. At each probed position, the deflection by the magnetic field is 

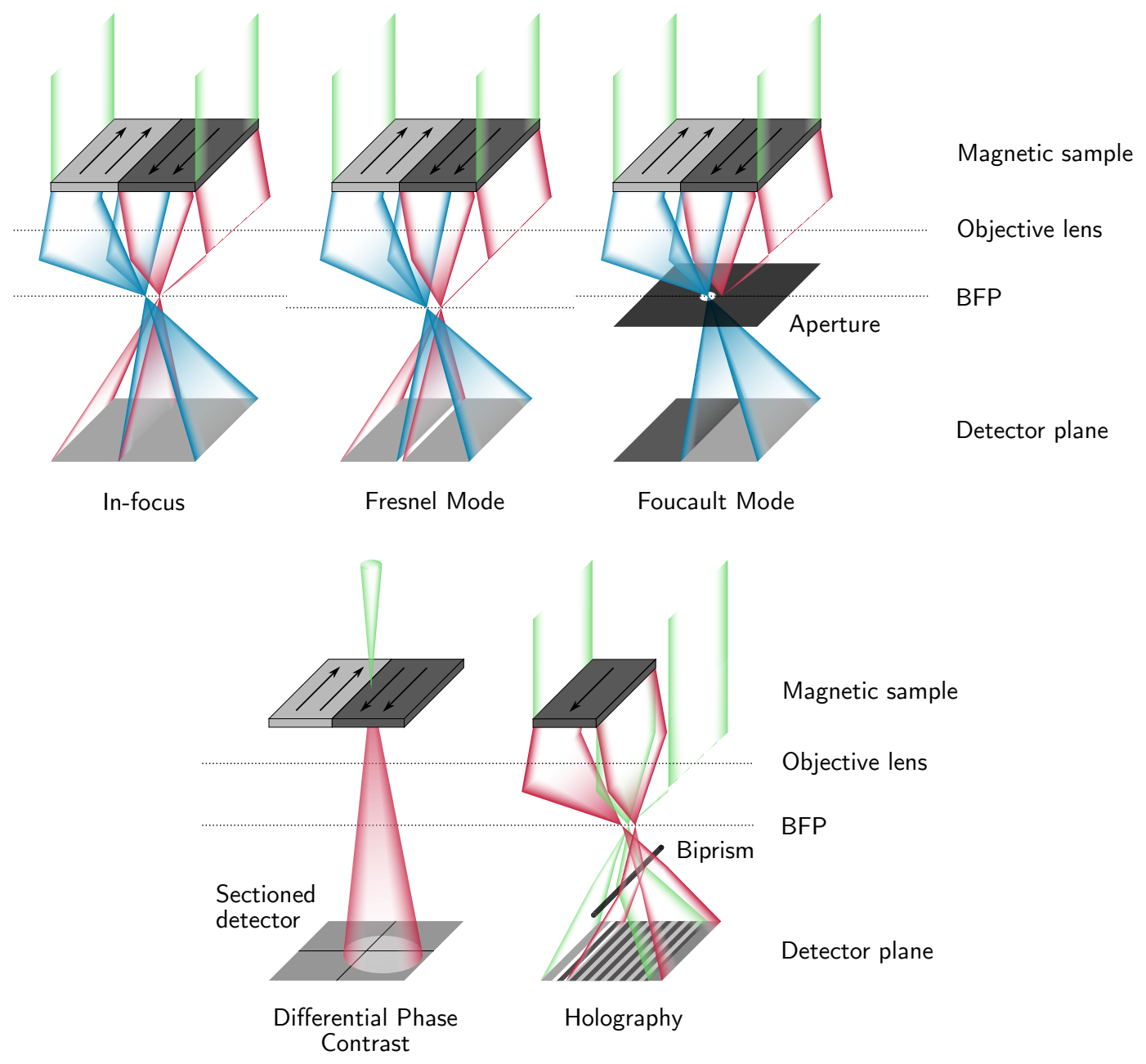

Fig. 2.7.: Schematic representation of methods for magnetic imaging in TEM. The green rays represent the incident or undeflected electron beam. The blue and red rays represent the beam deflected by the interaction with the in-plane magnetic field inside the sample. 
(a)

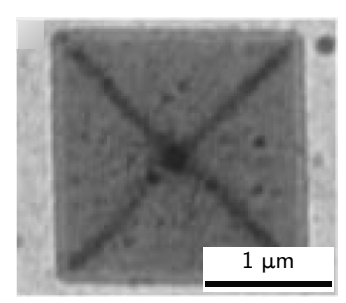

(b)

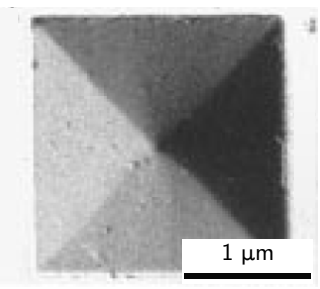

(c)
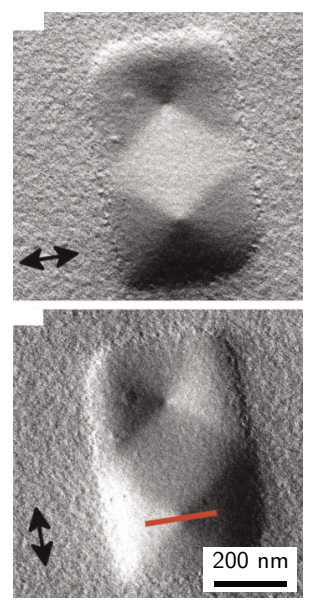

(d)

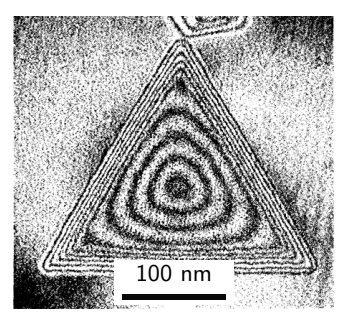

(e)

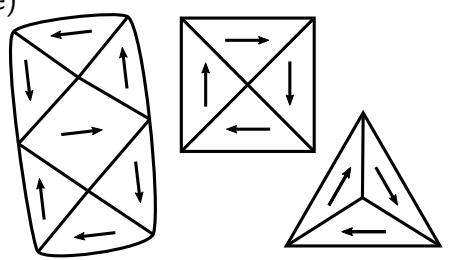

Fig. 2.8.: Illustration of the different modes of Lorentz microscopy applied to magnetic islands. (a) Fresnel mode. (b) Foucault mode. (c) Differential phase contrast showing the differential signal in two opposite sections of the detector (direction indicated by the arrows). (d) Reconstructed phase contour lines from an electron hologram. (e) Sketch of the magnetic domain configuration of the islands. (a,b) Reprinted from [78], with the permission of AIP Publishing. (c) Reprinted from [79], Copyright (2015), with permission from Elsevier. (d) Reprinted figure with permission from [74]. Copyright (1980) by the American Physical Society.

computed from the differential signal recorded in a sectioned detector, which is used for the image contrast.

- Electron holography: the probed area includes also an empty region, with no material. Before the image is formed, an electrostatic biprism is used to introduce an additional linear phase shift (deflection) on both the reference wave (going through vacuum) and the wave going through the material. The resulting interference pattern is used to reconstruct the phase of the wave function at the sample plane. The technique has various other applications than for mapping of magnetic fields [68, 72, 73]; for applications in magnetic materials, see, for example, Ref. [74-77].

\subsubsection{Contrast in Fresnel-mode Images}

Since we mainly use the Fresnel mode of Lorentz microscopy in this work, we now show how the phase of the exit wave function is transferred to the image contrast by the coherent aberration function in 2.13. The derivation is based in Ref. [62]. For small frequencies (the magnetic phase landscape is usually slowly varying with 
respect to the electron wavelength), and disregarding any coherence envelopes, the transfer function Equation 2.3 can be simplified to:

$$
T(\vec{q})=\cos \chi-\mathrm{i} \sin \chi \approx 1-\mathrm{i} \chi(\vec{q}),
$$

with

$$
\chi(\vec{q})=-\frac{\pi}{\lambda} \Delta f(\lambda q)^{2}
$$

After propagation through the electron-optical system, the initial exit wave function $\psi$ is modified to:

$$
\begin{aligned}
\psi_{i}(\vec{r}) & =\mathcal{F}^{-1}[T \mathcal{F}(\psi)] \\
& =\mathcal{F}^{-1}[\mathcal{F}(\psi)-\mathrm{i} \chi \mathcal{F}(\psi)] \\
& =\psi-\mathrm{i} \mathcal{F}^{-1}[\chi \mathcal{F}(\psi)] .
\end{aligned}
$$

By recognizing that $\mathcal{F}(\psi)$ is a function of spatial frequencies $q$, we can use the definition of a Fourier transform, and Equation 2.13 to compute the second term in Equation 2.14:

$$
\begin{aligned}
\mathrm{i} \mathcal{F}^{-1}[\chi \mathcal{F}(\psi)] & =-\mathrm{i} \pi \lambda \Delta f \mathcal{F}^{-1}\left[q^{2} \mathcal{F}(\psi)\right] \\
& =-\mathrm{i} \pi \lambda \Delta f \iint q^{2} \mathcal{F}(\psi) \mathrm{e}^{2 \pi \mathrm{i} \vec{q} \cdot \vec{r}} \\
& =-\mathrm{i} \pi \lambda \Delta f\left(-\frac{1}{4 \pi^{2}} \nabla^{2}\right) \iint \mathcal{F}(\psi) \mathrm{e}^{2 \pi \mathrm{i} \vec{q} \cdot \vec{r}} \\
& =\frac{\mathrm{i}}{4 \pi} \lambda \Delta f \nabla^{2} \psi
\end{aligned}
$$

Therefore, the wave function at the detector is:

$$
\psi_{i}(\vec{r})=\psi(\vec{r})-\frac{\mathrm{i}}{4 \pi} \lambda \Delta f \nabla^{2} \psi
$$

and the image intensity is calculated by taking the absolute value of $\psi_{i}$ :

$$
I=|\psi|^{2}+\frac{1}{16 \pi^{2}}(\lambda \Delta f)^{2}\left(\nabla^{2} \psi\right)^{2}-\frac{1}{4 \pi} \lambda \Delta f\left[\psi^{*}\left(\mathrm{i} \nabla^{2} \psi\right)+\psi\left(\mathrm{i} \nabla^{2} \psi\right)^{*}\right] .
$$


We can ignore terms in $\lambda^{2}$ here, again due to the comparatively long distances over which there are relevant modifications of the magnetic texture (and hence the phase of the exit wave function $\psi$ ). By explicitly writing the laplacian of $\psi=A_{0} \mathrm{e}^{\mathrm{i} \phi}$ as a function of amplitude $A_{0}$ and phase $\phi$ we get:

$$
\begin{aligned}
\mathrm{i} \nabla^{2} \psi & =\mathrm{i}\left\{A_{0} \nabla^{2}\left(\mathrm{e}^{\mathrm{i} \phi}\right)+2 \nabla A_{0} \cdot \nabla\left(\mathrm{e}^{\mathrm{i} \phi}\right)+\left(\nabla^{2} A_{0}\right) \mathrm{e}^{\mathrm{i} \phi}\right\} \\
& =\mathrm{i}\left\{A_{0} \mathrm{e}^{\mathrm{i} \phi}\left[-(\nabla \phi)^{2}+\mathrm{i} \nabla^{2} \phi\right]+2 \mathrm{ie}^{\mathrm{i} \phi} \nabla A_{0} \cdot \nabla \phi+\left(\nabla^{2} A_{0}\right)\left(\mathrm{e}^{\mathrm{i} \phi}\right)\right\} \\
& =\mathrm{e}^{\mathrm{i} \phi}\left\{\mathrm{i}\left[-A_{0}(\nabla \phi)^{2}+\nabla^{2} A_{0}\right]-\left[A_{0} \nabla^{2} \phi+2 \nabla A_{0} \cdot \nabla \phi\right]\right\} .
\end{aligned}
$$

Finally, substituting Equation 2.18 into Equation 2.17, we find the intensity distribution in a Fresnel-mode Lorentz micrograph to be:

$$
I(\vec{r})=|\psi|^{2}+\frac{1}{2 \pi} \lambda \Delta f\left[A_{0}^{2} \nabla^{2} \phi+2 A_{0} \nabla A_{0} \cdot \nabla \phi\right]
$$

It is clear from Equation 2.19 that the image contrast arises from the in-focus image superimposed with contrast arising wherever the curvature of the phase $\left(\nabla^{2} \phi\right)$ is non-zero and where there are simultaneous spatial variations in amplitude and phase. The amplitude $A_{0}$ of the exit wave function is mostly associated with mass-thickness contrast, which is especially relevant at boundaries between different materials (for instance, at the edges of nanoislands or holes in a thin film), where the transition is accompanied by a variation of the electrostatic phase shift. The term with $\nabla^{2} \phi$, however, is more interesting for magnetic materials. From this term, we find that there are contrast features arising wherever the curvature of the magnetic phase shift is non-zero, i.e., at magnetic domain walls or singularities. We note that this also holds for the electrostatic part of the phase shift, thus once again material boundaries will play a role. Equation 2.19 also shows that the contrast in Fresnel-mode Lorentz micrographs is linear in the defocus $\Delta f$. Moreover, the contrast is reversed between under-focus $(\Delta f<0)$ and over-focus $(\Delta f>0)$.

Equation 2.19 enables a qualitative description of the contrast arising at defocused micrographs of magnetic sample systems, demonstrating the origins of Fresnel contrast. However, real imaging conditions, including the influence of partially coherent illumination, require a more comprehensive description. Therefore, the 
simulations presented in Chapter 4 make use of the more elaborated description given in Section 2.2.2.

\subsubsection{Elements of Holography}

Holography was proposed by Gabor in 1949 for improving TEM resolution [80], but it was fully developed first in light optics shortly after the advent of the laser [81]. The technique requires high coherence illumination, and we use it in Chapter 3 for a quantitative evaluation of the electron beam coherence in our ultrafast TEM. Here, we briefly introduce electron holography and only the fundamental elements associated with its use within the scope of this thesis. The following is based on Ref. $[70,73]$.

A hologram is an interferogram recording both amplitude and phase of a wave, thus allowing a complete reconstruction of the original wave. The formation of a hologram in TEM is already sketched in Figure 2.7e, featuring only the essential elements. In holography, an interference region between a scattered wave $\psi_{o}$ from an object and a reference wave $\psi_{r}$ is obtained by employing an electrostatic biprism [82]. These are partial waves emitted from an extended, polychromatic electron source, and the contrast of the interference pattern $I$ in the overlapping region is therefore determined by the coherence properties of the source.

Information on the source coherence can be obtained by recording an empty hologram, i.e., without any sample. Accordingly, $\psi_{o}=\psi_{r}$, and the interferogram intensity $I$ is given by [70, 83]:

$$
I(x, y)=2 I_{0}\left[1+\mathcal{V} \cos \left(2 \pi q_{c} x+\bar{\epsilon}\right)\right]
$$

where $I_{0}$ is the intensity of the reference wave, $\mathcal{V}$ is the contrast of the interference fringes, $q_{c}=k \beta$ ( $\beta$ is the overlapping angle at the image plane $x y$ ) is the spatial carrier frequency, and $\bar{\epsilon}$ accounts for a lateral shift of the fringes. The fringes are considered to be parallel to the $y$-axis.

The fringe contrast $\mathcal{V}$ is given by:

$$
\mathcal{V}=\left|\mu_{s c}\right|\left|\mu_{t c}\right|,
$$


where $\mu_{s c}$ and $\mu_{t c}$ are the complex degrees of spatial and temporal coherence, respectively. Note that $\left|\mu_{s c}\right|$ and $\left|\mu_{t c}\right|$ are both smaller than 1. Further contrast reducing factors may be included in this product, such as instabilities (in the microscope or in the biprism) and the modulation transfer function of the detector [83].

The electron beam coherence is relevant for phase-contrast techniques in general, not just for holography. In the following, the beam coherence properties are introduced.

\subsection{Electron Beam Properties for Phase-contrast Imaging}

As detailed in Section 2.2.2, deviations from the ideal, point-like, monochromatic source restrict the transfer of phase information from the object to the image plane, thus reducing the capabilities of phase-contrast techniques. In this section, we introduce relevant concepts to describe the beam properties associated with these limitations.

The electron emitter in TEM has small areas $\delta a$ emitting quasi-monochromatic (deviations of $\delta \lambda$ ) radiation, i.e., coherent radiation. However tiny $\delta a$ and $\delta \lambda$ may be, the radiation emitted by each of these areas adds up incoherently. The resulting effects on the image intensity distribution can be phenomenologically represented by envelope functions (cf. Section 2.2.2). A rigorous derivation of an image formed by partially coherent sources can be found in Ref. [84] (for electron beams) and in Ref. [85] (for light).

In TEM, tools such as apertures and monochromators are employed to decrease the virtual source size and energy spread by filtering out the undesirable parts of the beam. This is achieved at the expense of the total beam current, but the trade-off between obtaining enough beam current to form an image and filtering to improve beam quality is ultimately limited by the intrinsic source properties.

In this section, a theoretical description of the beam is established first, after which the beam properties are introduced accordingly. The contents follow mostly Ref. [63, 84]. 


\subsubsection{Phase Space}

A useful theoretical framework to analyze the properties of electron beams can be established by describing them in the phase space $[63,84]$. To a classical system of $\mathrm{N}$ interacting particles corresponds a $6 \mathrm{~N}$-dimensional phase space $(\Gamma$-space), whose axes are determined by the 3 position-momentum pairs given by $x_{i}, p_{i x}, y_{i}, p_{i y}, z_{i}, p_{i z}$ of each particle $i$. Independent realizations (i.e., set of initial conditions) of the system are represented by a point in this $\Gamma$-space, and, accordingly, the ensemble of possible configurations defines a distribution of points in the $\Gamma$-space [86].

A volume element in phase space, given by $d V=\prod_{i=1}^{N} d x_{i} d p_{i x} d y_{i} d p_{i y} d z_{i} d p_{i z}$, is the density of points in the distribution. It can be shown that, upon canonical transformations such as temporal evolution, $d V$ remains invariant [86]. This result, known as Liouville's theorem, is consequently a conservation law for the total volume of the distribution.

An electron beam consisting of several electrons can be represented by an ensemble of single electron emission events. The emission position, momentum and time are statistically distributed according to the electron emitter properties. Therefore, electron beams are described by a finite distribution in phase space. The consequences of Liouville's theorem for the beam evolution will be dealt with in the following section.

\subsubsection{Emittance and Brightness}

Brightness and emittance are useful quantities for describing the quality of the electron beams $[63,84]$. In the following, we briefly present these quantities and discuss conditions for their conservation with beam propagation in TEM.

As mentioned in the previous section, the representation of an electron beam in phase space carries information about the electron source. Assuming $z$ is the beam axial direction and that the electron-electron interactions can be expressed as a mean-field, we can analyze the beam in the 4-dimensional subspace defined by the transversal coordinates $x, p_{x}, y, p_{y}$. The quantities describing the beam properties are then:

- Hyperemittance $E^{*}$ : the volume of the distribution in the 4D-subspace [84]; 
- Differential brightness $B$ : the local current density in the 4D-subspace [87].

Note that the emittance is an overall property of the beam, whereas the brightness is defined over a portion of it. The conservation law stated by Liouville's theorem can be extrapolated to the beam emittance and brightness in TEM (i) if electron interactions are negligible or if they can be described by a mean field [84]; and (ii) as soon as the beam has passed the last aperture and is subject only to the action of the microscope lenses and deflectors (in good approximation, acting on the beam only with linear forces) [87].

Before moving on, it is necessary to introduce the concept of trace space, which is defined by the intersection of the particle trajectories (i.e., position $x, y$ and slope $x^{\prime}=d x / d z, y^{\prime}=d x / d z$ coordinates) with a given plane $z=$ constant and usually transverse to the electron beam direction. Whenever the beam has two planes of symmetry (generally taken as $x z$ and $y z$ ), orthogonally arranged relative to each other and to the optical axis $z$, the motions $x(z)$ and $y(z)$ are decoupled and one can define the two-dimensional emittances $\epsilon_{x}$ and $\epsilon_{y}$ by projecting $V_{4 D}$ onto the corresponding subspaces [84].

Since the volume $V_{4 D}$ in trace space may have a complicated shape, a pragmatic expression for the emittance is given by the normalized root-mean-square (rms) emittance for $x$ (and analogously for $y$ ) [63, 87]:

$$
\tilde{\epsilon}_{n x}:=\beta \gamma \sqrt{<x^{2}><x^{\prime 2}>-<x x^{\prime}>^{2}},
$$

where $\left\langle x^{2}>,\left\langle x^{\prime 2}>\right.\right.$ and $\left\langle x x^{\prime}\right\rangle^{2}$ are the moments of the electron distribution in trace space, and $\beta$ and $\gamma$ are the usual relativistic parameters. The two first terms are the standard deviations of position and slope within the beam, whereas the third reflects correlations between position and slope in planes $z$ out of the beam waist. The definition in Equation 2.22 is practical because the correlation $\left\langle x x^{\prime}\right\rangle^{2}$ vanishes at the beam waist, and the emittance can be calculated by measuring the rms width $\sqrt{\left.<x^{2}\right\rangle}$ and rms divergence $\sqrt{\left\langle x^{\prime 2}\right\rangle}$. 
(a)

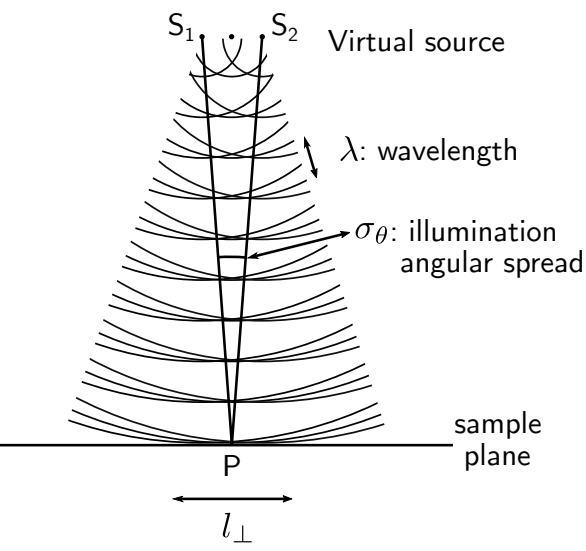

(b)

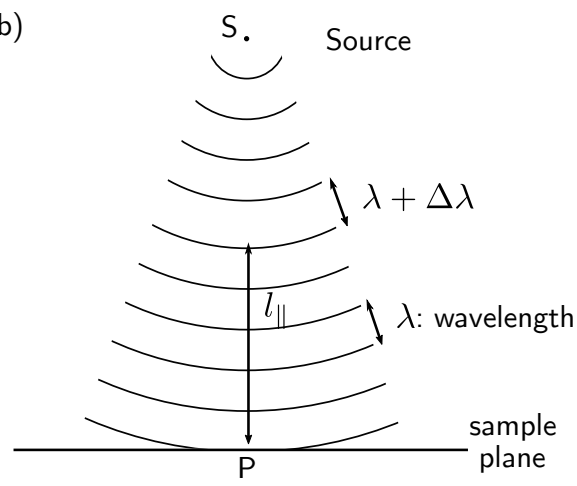

Fig. 2.9.: Diagrams showing the definition of (a) spatial and (b) temporal coherence lengths.

The brightness can also be defined in the trace space by writing the current within the volume element $d x d y d x^{\prime} d y^{\prime}$ as $d^{4} I$ [87]:

$$
B=\frac{d^{4} I}{d x d y d x^{\prime} d y^{\prime}},
$$

which, for a Gaussian distribution in trace space, results in a normalized peak brightness given by [87]:

$$
B_{n p}=\frac{1}{8 \pi^{3}} \frac{2 I}{\tilde{\epsilon}_{n x} \tilde{\epsilon}_{n y}} .
$$

Being characteristic figures for the beam quality, brightness and emittance relate with the coherence properties of the electron beam, which will be introduced in the next section.

\subsubsection{Coherence}

For an extended, polychromatic source as a TEM emitter, the beam coherence is associated with the non-local correlations between waves emitted at different points $S_{1}$ and $S_{2}$ (spatial coherence), and with the local fluctuations in amplitude and phase of the radiation emitted from each source point (temporal coherence) [84, 85]. The former leads to the concept of a region of coherence around any point $P$ of the wave field, in which wavefronts can be defined (see Figure 2.9a), while the later sets a characteristic coherence time within which the phase at $P$ varies linearly with time (see Figure 2.9b). 
It is reasonable to define coherence lengths for the concepts of temporal and spatial coherence. Temporal coherence (see Figure 2.9b) is operative along the optical axis, and is characterized by a longitudinal coherence length $[73,85]$ :

$$
l_{\|}=\frac{\lambda^{2}}{\Delta \lambda}
$$

where $\lambda$ is the mean electron wavelength and $\Delta \lambda$ its corresponding uncertainty.

On the other hand, spatial coherence is connected to directions perpendicular to the optical axis (see Figure 2.9a). The transverse coherence length is usually defined as the diameter of the illuminated circular area for which the degree of spatial coherence (given by the Van Cittert-Zernike theorem) departs $12 \%$ from the ideal unitary value $[84,85]$ :

$$
l_{\perp}=0.16 \frac{\lambda}{\sigma_{\theta}}
$$

In this equation, $\sigma_{\theta}$ is the angular diameter of the source region between the points $S_{1}$ and $S_{2}$, when viewed from $P$ (see Figure 2.9a), and can be replaced by the root-mean-square (rms) angular spread.

The degree of spatial coherence $\left|\mu_{s c}\right|$ can be defined from the relative size of the coherent region and the total illuminated area with diameter (or rms beam width) $\sigma_{r}[85]:$

$$
\left|\mu_{s c}\right|=\frac{l_{\perp}}{\sigma_{r}}
$$

A last useful quantity is the coherent electron current $I_{c o h}$, which is the beam current available in the region of coherence, and is given by [70]:

$$
I_{c o h}\left(\left|\mu_{s c}\right|\right)=-\ln \left(\left|\mu_{s c}\right|\right) B_{n p},
$$

where $B_{n p}$ is the normalized peak brightness of the beam. Because of the multiplication by $B_{n p}$, the coherent current is independent of the acceleration voltage. Note that: (i) the coherent electron current is zero for full coherence $\left(\left|\mu_{s c}\right|=1\right.$ ), and (ii) guns with high brightness (like Schottky emitters or cold field emitters) are more convenient for phase-contrast techniques.

The concepts presented in this section are key to the critical evaluation of the unique capabilities of the Göttingen UTEM for phase-contrast imaging. In particular, it 
provides a theoretical background for the discussions on the challenges for the implementation of fs-Lorentz microscopy in Chapter 3.

\subsection{Ultrafast Transmission Electron Microscopy}

Time-resolution in Transmission Electron Microscopy (TEM) is in principle readily attainable by using conventional video cameras equipped with a proper electron detector, but the response times of even the fastest detectors are limited to the millisecond range. Since several relevant physical processes taking place at the short length scales accessible by electron microscopy occur in a much faster timescale (from the nanosecond down to the femtosecond range), it is essential to develop faster time-resolved TEM. This is a reason for the ever increasing number of research groups working on this topic [23-26, 28-30, 88-91]. Approaches achieving the highest temporal resolution build upon the idea of employing short laser pulses for establishing concepts of laser-driven electron emitters:

- The first approach, known as Dynamic Transmission Electron Microscopy (DTEM), has single-shot probing capabilities. Early work was pioneered by Bostanjoglo at the Technical University of Berlin [92] and further developed at the Lawrence Livermore National Laboratory $[93,94]$. In DTEM, a train of nanosecond electron bunches (containing typically $10^{7}-10^{8}$ electrons each [95]) is generated by optically stimulated photoemission and is subsequently used to probe a sample at which a dynamical process has been triggered by an excitation pulse (usually synchronized with the first electron bunch).

- The second approach, which we call Ultrafast Transmission Electron Microscopy (UTEM), makes use of stroboscopic electron illumination. Pioneering work was done by Petrov at the Moscow State University [12] and by Bostanjoglo, reaching nanosecond resolution by chopping the electron beam using a double-plate capacitor [11]. Temporal resolution was pushed down to the sub-picosecond range by the group of Zewail at Caltech $[23,96]$ by employing laser-driven photoemission.

DTEM and UTEM differ fundamentally in two points. First, UTEM can only probe reversible phenomena, while DTEM has also the ability to probe irreversible dynamic 
processes. On the other hand, better temporal resolution can be achieved in UTEM, since shorter laser pulses cannot produce the high charge pulses as needed for DTEM $^{1}$.

As for spatial resolution capabilities, a high number of electrons per pulse imposes severe constraints to the transverse coherence properties of the pulsed electron beam. In DTEM, the resolution limit was initially estimated from noise statistics to be about $48 \mathrm{~nm}$ [92], but it it has since been shown that about $10 \mathrm{~nm}$ can be achieved by advancements in the electron source and the lens system [95]. In UTEM, measuring in the single-electron-per-pulse regime is still feasible, and spatial resolution similar to non-time-resolved TEM has been demonstrated for different UTEM instruments $[28,96]$. A further discussion on the detrimental effects of a high number of electrons per pulse on beam coherence properties is provided in Section 2.4.3.

\subsubsection{Previous Approaches for Magnetic Imaging}

Relatively slow dynamics of magnetic features occurring in the second to millisecond range have been addressed by using video cameras. The first recordings were performed for superconductors by the group of Tonomura at the Hitachi Ltd. Laboratories, both in electron holography [98] and Lorentz microscopy [99]. For magnetic materials, domain formation in films supporting perpendicular magnetization [100] and the response of skyrmion lattice dynamics to temperature variations [101-103] was captured using Lorentz microscopy.

As mentioned in the previous section, the achievable time resolution is improved in stroboscopic approaches. The first stroboscopic experiments performed by the groups of Petrov at the Moscow State University [12] and of Bostanjoglo at the Technical University of Berlin were in Lorentz microscopy, reaching nanosecond time resolution [11] (see Figure 2.10). Only 30 years later, magnetic dynamics was imaged using UTEM, with a time resolution in the nanosecond $[16,104]$ down to the picosecond range [18]. Figure 2.11 displays Lorentz micrographs of optically

\footnotetext{
${ }^{1}$ Electron-pulse compression schemes are not discussed here (see, for example, Ref. [95, 97]). Although providing a valid alternative to enhance temporal resolution in DTEM (and also in UTEM), they cannot counterbalance the non-reversible effects of high electron densities on the electron pulse energy width.
} 

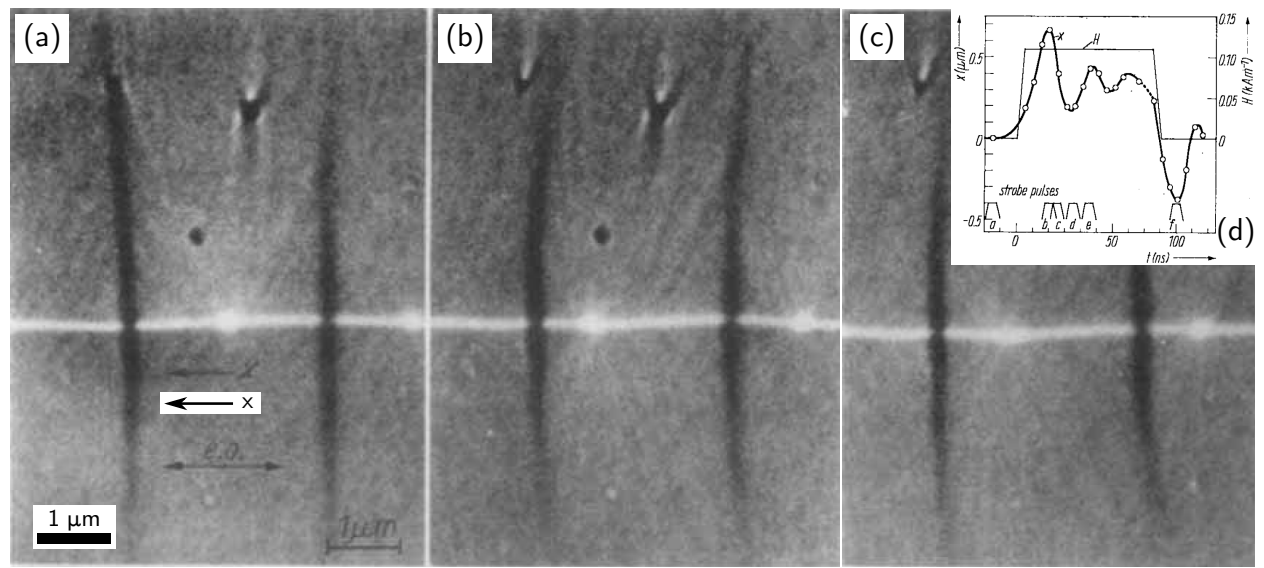

Fig. 2.10.: Pioneering stroboscopic Lorentz micrographs of 40-50-nm thick NiFe films (a-c) obtained by chopping a continuous electron beam using a double-plate capacitor. A magnetic field pulse $H$ along the $x$-direction marked in (a) triggers the motion of the Bloch lines (singularities at the domain wall producing a bright-spot-like contrast feature). In (d), the displacement is followed with time resolution given by the pulse duration ( $\geq 1 \mathrm{~ns}$ ). The delay times relative to the excitation pulse $H$ for each micrograph are marked as $(\mathrm{a}, \mathrm{b}, \mathrm{c})$ in the plot (d). Reproduced with permission from [11]. Copyright (C) 1980 WILEY-VCH Verlag GmbH \& Co. KGaA.

triggered domain wall (DW) dynamics recorded by the UTEM instruments of Zewail group at Caltech and Flannigan group at the University of Minnesota.

We selected the images in Figures 2.10 and 2.11 for further comparison to the achieved spatiotemporal resolution in this work (cf. Chapter 5). Note that the DWs in Figure 2.11 can be resolved with about $300 \mathrm{~nm}$ and $1 \mu \mathrm{m}$, and with lower contrast than in Figure 2.10. In spite of that, temporal resolution was significantly improved. All the works $[11,16,18,104]$ used microscopes equipped with thermionic sources $\left(\mathrm{LaB}_{6}\right)$, which, for non-time resolved TEM, provides electron beams with reduced coherence properties in comparison to field-emission based sources [62, 69]. Since magnetic imaging relies on phase contrast capabilities, UTEM employing high coherence emitters (as the Göttingen UTEM) present favorable conditions for investigating magnetic dynamics at the nanoscale.

\subsubsection{The Göttingen UTEM}

Here, the UTEM instrument we recently developed in Göttingen is described, along with the specific technical details for implementing time-resolved Lorentz microscopy within this thesis. More details on the instrumentation and on various applications are found in Ref. [28]. 

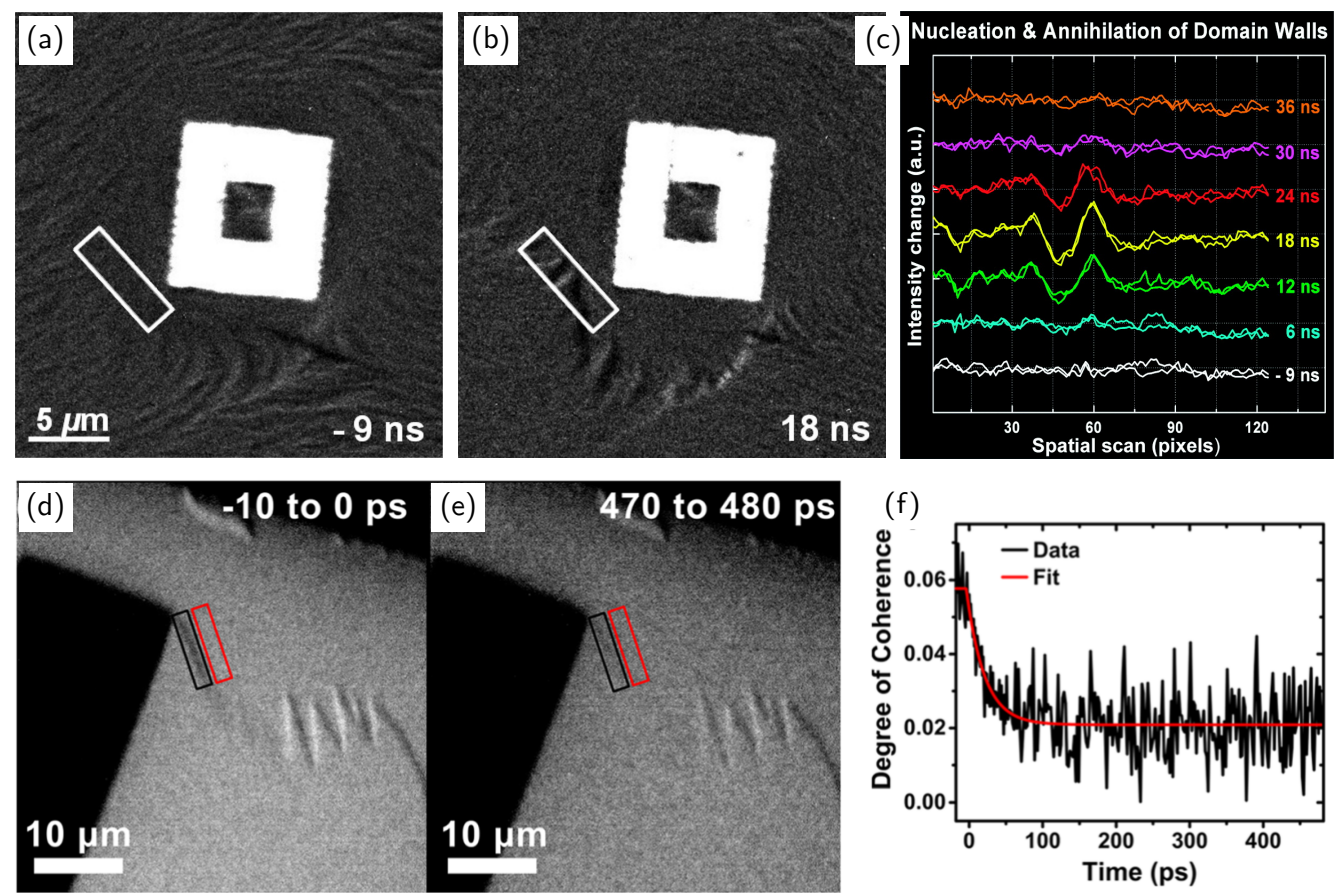

Fig. 2.11.: Domain walls (DWs) in optically excited magnetic films investigated by Ultrafast Transmission Electron Microscopy using electron pulses photoemitted from thermionic sources. (a,b) Time-resolved Lorentz micrographs (Fresnel mode) close to a milled structure in a $\mathrm{Ni} / \mathrm{Ti}(30 \mathrm{~nm} / 30 \mathrm{~nm})$ film exposed to pulsed laser excitation (532-nm wavelength, $6.4 \mathrm{~mJ} / \mathrm{cm}^{2}$ at 1-kHz repetition rate, 10-ns temporal duration). (c) Temporal evolution of line intensity profiles corresponding to the rectangular regions shown in (a,b), evidencing the nucleation and annihilation of the DWs following laser excitation. (d-e) Sum of time-dependent Lorentz micrographs (Fresnel mode) close to a Pt island on 35-nm thick FePt film exposed to pulsed laser excitation (515-nm wavelength, $1 \mathrm{~mJ} / \mathrm{cm}^{2}$ at $5-\mathrm{kHz}$ repetition rate, $700-\mathrm{fs}$ temporal duration). (f) The temporal evolution of DW contrast (labelled "degree of coherence" in the source paper), measured from the image intensities within the black and red rectangles depicted in $(\mathrm{d}, \mathrm{e})$, evidences an exponentially decaying demagnetization with 24-ps time constant. (a-c) Reprinted with permission from [16]. Copyright (2010) American Chemical Society. (d-f) Reprinted from [18], with the permission of AIP Publishing. 
The Göttingen UTEM is an implementation of a laser-pump/electron-probe experimental setup based on a transmission electron microscope and a femtosecond laser system (Figure 2.12). The microscope (JEOL JEM-2100F) was modified for realizing sample optical excitation (pump) and pulsed electron emission (probe). For both pump and probe, we use a pulsed laser beam (800-nm central wavelength, 250/500-kHz repetition rate, 50-fs pulse duration) from an amplified femtosecond laser system (Coherent RegA 9040 regenerative amplifier seeded by a Coherent Vitara Ti:sapphire oscillator). The laser beam is split in two parts, one being frequencydoubled by a $\beta$-barium borate (BBO) crystal and focused to about a $20-\mu$ m-diameter full-width-at-half-maximum (FWHM) spot by a movable lens onto the microscope electron emitter (a zirconium-oxide covered tungsten tip, see top inset of Figure 2.12) in a Schottky electron gun. The electron pulses that are generated by the laser pulses are subsequently coupled to the accelerator stage and microscope column. The second part of the split laser beam is used for the sample excitation, guided along a path which allows for pulse duration control (by the introduction of glass bars), fluence control (by the use of a variable attenuator) and polarization control (by the use of $\lambda / 2$ - and/or $\lambda / 4$-waveplates). The pump laser beam is focused to about a 50 - $\mu$ m-diameter FWHM spot onto the sample by a movable lens, reaching the sample at a $55^{\circ}$ angle relative to the microscope optical axis (see bottom inset of Figure 2.12). The timing between laser-pump and electron-probe pulses (at the sample plane) is controlled by a motorized delay stage.

For real-space and reciprocal-space measurements, the microscope is equipped with a digital detector (Gatan UltraScan 4000). In addition, an electron energy spectrometer (Gatan Enfinium) attached to the bottom of the microscope column enables electron-energy-loss measurements. In this thesis, we make use of the energy spectrometer only for the measurement of the electron pulse duration by electron-photon cross-correlation [27, 28, 105] (cf. Section 3.2.2). For all other experiments, we use the Low Magnification mode (shortly LowMag) of the UTEM, for which two of the microscope lenses are turned off (greyed out in Figure 2.12). One of them is an immersion-type objective lens, wherein the sample is located; and hence high magnification is not possible in this mode. On the other hand, since all TEM lenses are electromagnetic, the sample now sits in a field-free region, which is 


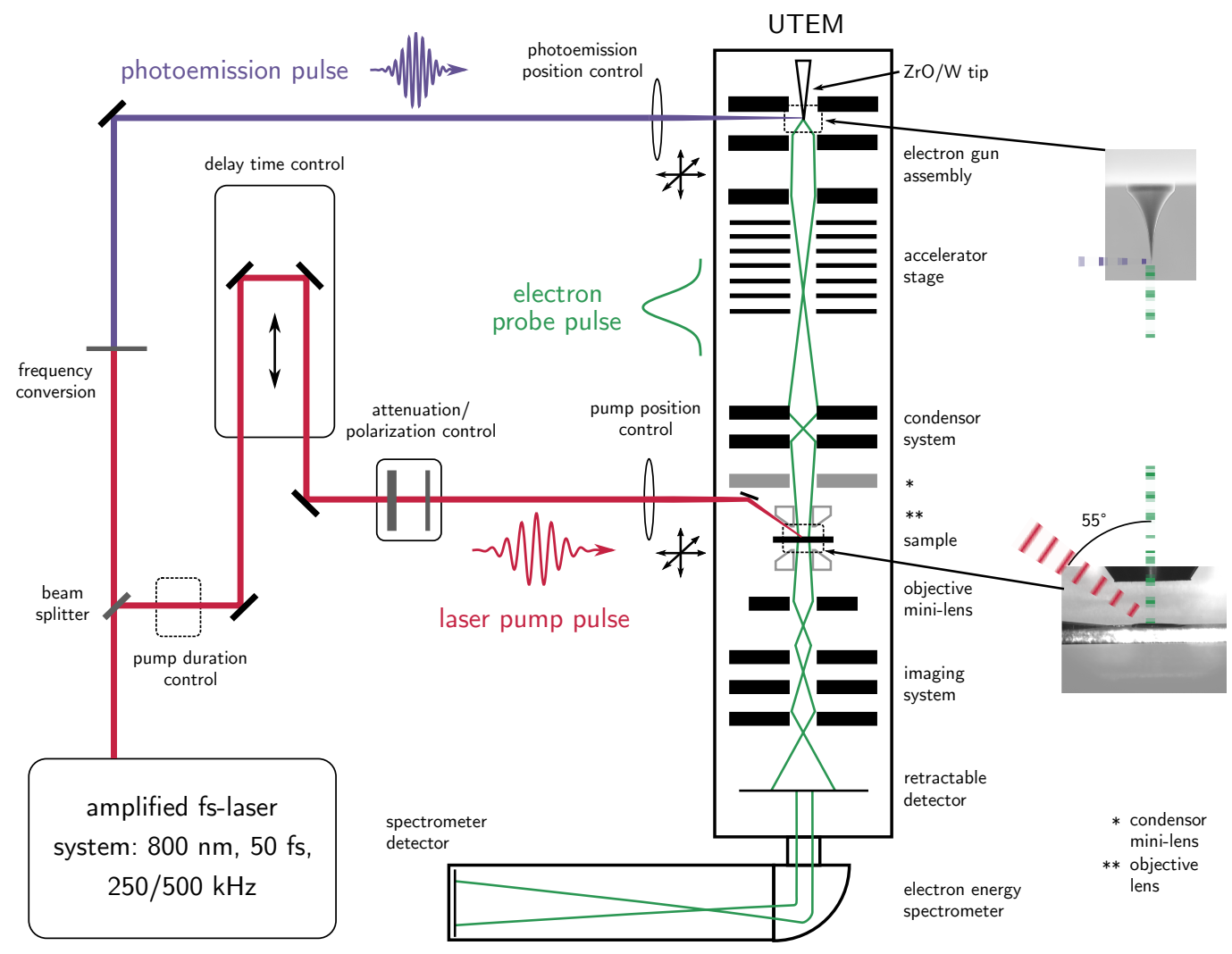

Fig. 2.12.: Scheme of experimental setup. A pulsed laser beam (50-fs duration, $800-\mathrm{nm}$ wavelength, $250 / 500-\mathrm{kHz}$ repetition rate) is split and subsequently used for sample excitation (pump) and for initiating the electron pulses (probe) after double-frequency conversion. The electron pulses are generated by singlephoton photoemission from a heated $\mathrm{ZrO} / \mathrm{W}$ Schottky-field emitter (top inset) with tunable emission current and pulse duration. The pump beam reaches the sample at a $55^{\circ}$ angle relative to the microscope optical axis (bottom inset). The delay time between pump and probe pulses is defined by a variable pump beam path length (mirrors mounted on a motorized stage). 
a desirable environment for preserving the magnetic microstructure of the sample $[62,69]$.

The corresponding ray diagram in LowMag is depicted in Figure 2.12 (green lines in the microscope column, only down to the retractable detector). A well-collimated illumination electron beam is formed by two condensor lenses, and a low magnification image is formed by the action of the objective mini-lens, located further down the column (contrary to the immersion objective lens), and additionaly magnified by three lenses comprising the imaging system. Within this thesis, magnetic contrast is obtained in the Fresnel mode of Lorentz microscopy by changing the objective mini-lens current (cf. Section 2.2.1, and Ref. [62, 66, 69]).

\subsubsection{Coherence of Ultrashort Electron Pulses}

In this section, we briefly introduce the mechanism of photoemission utilized in the Göttingen UTEM concept, and discuss the achievable coherence properties.

We operate the laser-driven Schottky photoemitter in a single-photon-photoemission (1-PPE) regime, for which the emitted electron current scales linearly with the photoemission laser intensity. In a Schottky electron gun, a heated emitter tip (W) is placed in a electrostatic electrode assembly allowing for heat-assisted field emission $[69,84]$. The tip is coated by a thin ZrO layer, which reduces the work function. For conventional (not laser-driven) operation, this type of electron gun is among the best ones regarding brightness and energy spread, surpassed only by cold field emitter guns $[62,69]$.

For pulsed electron beams containing few or more electrons strongly bunched in time (as for femtosecond electron pulses), stochastic effects analogous to those originally observed for high current density continuous beams [106] may limit the beam quality. Coulomb interactions between the electrons contribute a mean field inducing reversible deformations in the phase space (see Section 2.3.1). After subtracting the mean Coulomb field, however, the residual stochastic variations in local charge distributions (resulting here from pulse-to-pulse fluctuations) induce an anomalous broadening of the phase space distribution [84], which is irreversible. The associated shifts in the longitudinal velocities manifest as a broadening of the energy distribution (Boersch effect [106]), whereas the shifts in the transverse 
velocities manifest as a broadening of the beam diameter (trajectory displacement effect [107]). As a result, the pulsed beam brightness/emittance and energy spread are affected by the number of electrons per pulse.

We have already demonstrated that our modified Schottky photoemitter can achieve a brightness and an energy spread similar to conventional operation, enabling us to perform imaging, diffraction and spectroscopy measurements using the photoelectron beam with comparable quality to a conventional electron beam (Figure 2.13, reproduced from Ref. [28]). In addition, a systematic investigation of the effects of stochastic Coulomb interactions on the ultrashort electron pulses obtained in the UTEM has recently established the impact of the electron pulse charge on its transverse and longitudinal coherence properties in a quantitative manner [108]. The stochastic effects are only found in the transverse properties (emittance), while the spectral growth is governed by reversible space-charge induced temporal broadening. Such an investigation is essential for developing strategies to tailor the electron pulse properties to specific experimental requirements. We note that the stochastic effects are present only within a few micrometers after emission [108], and the mean-field approximation is sufficient for further propagation along the microscope column, which ensures emittance conservation (see Section 2.3.2).

In conclusion, the Göttingen UTEM photoemitter delivers electron pulses with record properties among the ultrafast electron microscocopy approaches worldwide, reaching 9-Å beam diameter, 200-fs pulse duration and 0.6-eV energy width [28]. The excellent emittance of our laser-driven Schottky emitter reaches values as low as $1.71 \mathrm{~nm} \cdot \mathrm{mrad}$ at $200 \mathrm{kV}$ operation and in the single-electron-per-pulse limit [28], which is only 1 order of magnitude larger than the minimum emittance limited by the uncertainty principle (in the order of $0.193 \mathrm{~nm} \cdot \mathrm{mrad}$ ). As a comparison, the same UTEM electron gun (at $120 \mathrm{kV}$ and fixed emitter electrodes voltages) provides an electron beam with emittance of $6.8,8$ and $8.2 \mathrm{~nm} \cdot \mathrm{mrad}$ when operated for conventional Schottky emission, cw-laser-driven photoemission and fs-laser-driven photoemission (low pulse charges), respectively [108]. For increasing pulse charge, a slight degradation of the coherence properties is observed [108]. 

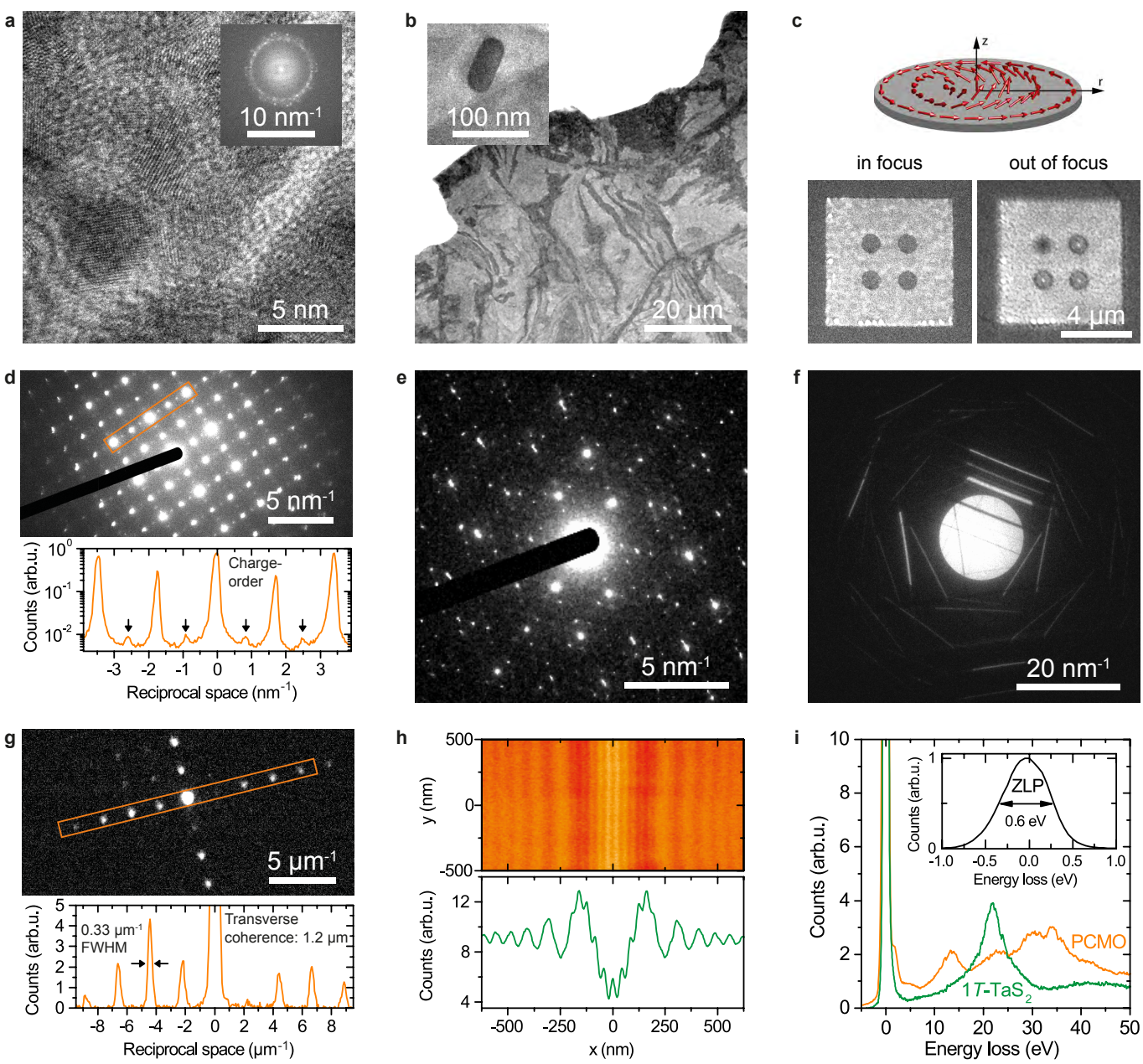

Fig. 2.13.: Exemplary experimental results achievable with the current status of the UTEM instrument. (a) High-resolution TEM (HRTEM) micrograph of Au/Pd particles on an amorphous carbon film. Visible lattice planes with $2-\AA \AA$ spacing demonstrate the resolution capabilities of the modified instrument (here: using thermal electron emission). Inset: Fourier transform of a four times larger sample region. (b-i) Measurements acquired with photoelectron beams (typical acquisition times 5-60 s) and at an electron energy of $120 \mathrm{keV}$. (b) Bright-field image of an ultramicrotomed $50 \mathrm{~nm}$ thin sample of $1 \mathrm{~T}-\mathrm{TaS}_{2}$ showing bending contrast of the thin-film membrane. Close-up: drop-casted gold nanorod on the sample surface. (c) Lorentz imaging provides magnetic contrast in UTEM as demonstrated for permalloy islands on a silicon nitride support (see also Ref. [53]). Out-of-focus image reveals the existence of a magnetic vortex in each of the four islands (visible as black and white features, respectively, depending on vortex orientation). Magnetic structure of a single vortex is schematically depicted in the upper panel. (d) Diffraction pattern of the charge-ordered phase of an ion-polished PCMO $\left(\mathrm{Pr}_{0.7} \mathrm{Ca}_{0.3} \mathrm{MnO}_{3}\right)$ plan view sample. Weak superstructure spots are visible halfway between the lattice reflections. (e) Diffraction pattern of the nearly commensurate charge density wave (NC-CDW) phase of $1 \mathrm{~T}-\mathrm{TaS}_{2}$. The first-order NC-CDW diffraction spots are hexagonally arranged around structural reflections. (f) Convergent beam electron diffraction (CBED) pattern of an exfoliated $100 \mathrm{~nm}$ thick single-crystalline graphite flake. (g) High dispersion diffraction pattern of a $463 \mathrm{~nm}$ spaced grating replica, demonstrating $1.2-\mu \mathrm{m}$ transverse coherence lengths. (h) Electron hologram obtained using a Möllenstedt biprism at a filament voltage of $9 \mathrm{~V}$, emphasizing the photoelectron coherence properties achievable in the UTEM. (i) Electron energy loss (EEL) spectra of $1 \mathrm{~T}-\mathrm{TaS}_{2}$ and PCMO. Inset: zero-loss peak (ZLP) with a FWHM of $0.6 \mathrm{eV}$. Reprinted from Ref. [28]. 


\section{Aspects and Challenges of fs-Lorentz Microscopy}

LORENTZ MICROSCOPY is a phase contrast technique, as explained in the previous chapter. In order to image magnetization dynamics by time-resolved electron microscopy, it is hence essential that the hundreds-of-femtoseconds illuminating electron pulses exhibit good coherence properties, conditions met in the Göttingen UTEM. As a pump-probe experimental technique, the processes to be investigated must be reversible, what poses a constraint for potential sample systems.

This Chapter describes details on establishing illumination conditions suitable for time-resolved Lorentz imaging in UTEM and on the development of the investigated magnetic sample. In Section 3.1, the preparation details of the magnetic sample system are described, and the importance of an optimized sample design for pumpprobe experiments is illustrated. Section 3.2 demonstrates the proper coherence properties for fs-Lorentz microscopy, comparing results with conventional Lorentz micrographs. In addition, the characterization of the utilized electron pulses is shown. Finally, in Section 3.3, strategies to evaluate the low-dose fs-Lorentz micrographs are presented. Parts of the results of this chapter were recently published in Ref. [109] and in Ref. [28].

\subsection{Sample Design for fs-Lorentz Microscopy}

Here, we describe the samples prepared for initial fs-Lorentz microscopy experiments in UTEM. We chose a well established sample geometry previously investigated in non-time resolved TEM: a magnetic disc of hundreds-of-nanometer diameter [110, 111]. It constitutes an ideally suited model system for verifying and characterizing the capabilities of the Göttingen UTEM instrument for Lorentz imaging.

In this spatially confined magnetic system, the spins tend to align parallel to the edges in order to form flux closure domains, which minimizes stray fields. The 
equilibrium magnetic distribution formed is a vortex, for which the magnetization is oriented in plane, curling around the center of the disc. Close to the center, the magnetization turns to an out-of-plane direction (cf. 4.8a), forming the vortex core with typical diameter of about $10 \mathrm{~nm}$ as calculated from variational approaches [38] or micromagnetic simulations $[110,112]$.

A vortex in a nanodisc is a very stable magnetic structure, which makes it a suitable candidate for pump-probe experiments, since a well defined initial state upon arrival of each pump pulse is essential.

\subsubsection{Preparation Details}

The magnetic nanoislands were prepared on a conventional TEM substrate: a 50-nm silicon nitride membrane. The substrate is practically transparent for both electrons and the 800-nm pump laser. In addition, silicon nitride films are amorphous, which usually means they do not contribute strongly to the phase contrast in transmission electron microscopy.

The magnetic material, permalloy, is an alloy consisting of $80 \% \mathrm{Ni}$ and $20 \% \mathrm{Fe}$. It has well-known magnetic properties, and fabrication of permalloy structures has been reported in the literature $[39,110,111,113-115]$. For preparing the magnetic islands, we used two different methods, namely focused-ion beam (FIB) patterning and electron-beam (e-beam) lithography, which are both laid out in the following.

The FIB-patterning method is sketched in Figure 3.1 (top row). A continuous permalloy film with a thickness of about $30 \mathrm{~nm}$ was prepared on the substrate by thermal evaporation of a permalloy lump. The islands are formed by removing magnetic material using a focused-ion beam. The resulting islands (of several shapes and sizes) are surrounded by the remaining permalloy film within a few- $\mu \mathrm{m}$ distance. The electron-beam lithography process is depicted in Figure 3.1 (bottom row). First, a roughly 100-nm thick resist (PMMA) layer is prepared on the substrate by spin-coating. The layer is then exposed to an electron beam on the regions to be later occupied by the islands. The resist is subsequently developed by immersion in a solvent, forming a positive mask. Next, a 20-nm thick layer of permalloy is evaporated by electron-beam evaporation. Finally, the remaining resist is removed 


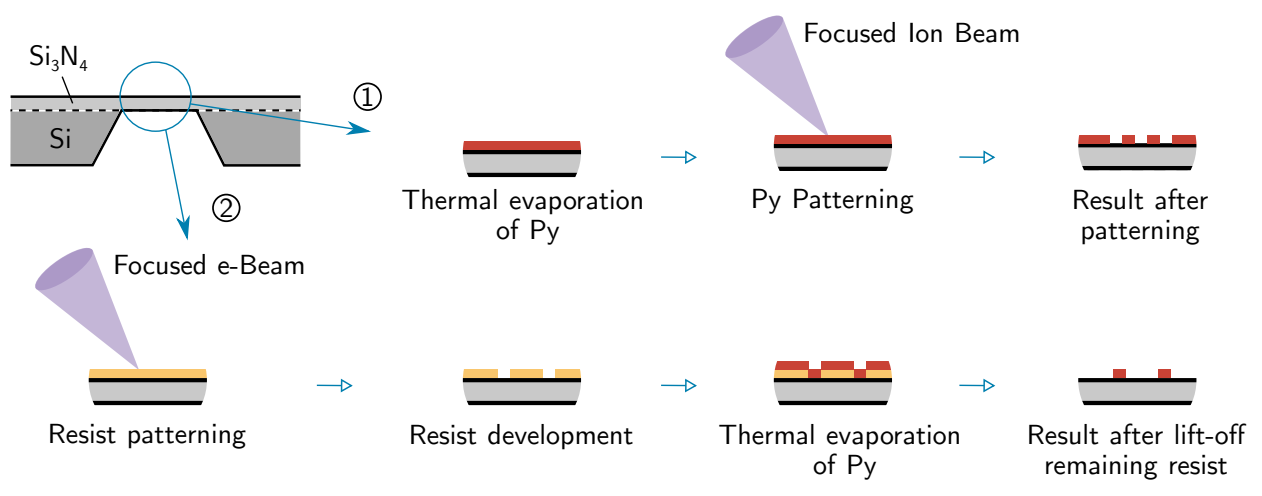

Fig. 3.1.: Preparation of magnetic permalloy (Py) nanoislands. Top row: FIB-based method. Bottom row: e-beam lithography and lift-off.

by immersion in acetone, lifting the unwanted parts of the permalloy. The positive mask can be prepared in such a way that the resulting islands can be arranged to in any desired pattern or they can even be isolated.

In Figure 3.2, we show three of the prepared nanoislands configurations. On the overview images, one observes the first qualitative differences between the resulting islands obtained by both methods. Notably, the FIB-patterning may lead to a few holes in the substrate, a nonexistent side effect in e-beam lithography. More relevant distinctions are visible in Lorentz micrographs of a smaller region containing 1- $\mu \mathrm{m}$ diameter disc(s) of each arrangement (see insets). In particular, the presence of circular contrast fringes immediately outside the disc edges can only be seen for the lithographically prepared discs, which indicates an improved edge quality obtained by this method. Inside the discs, the bright or dark spot in the center evidences the formation of the vortex state in all prepared discs (see Section 2.2.4).

A further important difference between the samples prepared by both methods is the crystallinity of the resulting nanoisland. The evaporation typically yields a polycrystalline permalloy film with crystal grains smaller than $10 \mathrm{~nm}$. However, the exposure to the ion beam modifies the crystalline structure of the FIB-patterned nanoislands. Specifically, the crystallites grow to about $30-40 \mathrm{~nm}$, becoming larger than the vortex core itself. These relatively large grains are undesirable for Lorentz microscopy, since grain boundaries always introduce phase contrast features due to local changes on the "effective" mean inner potential. The additional contrast may blur the phase contrast arising from the magnetic texture (example micrographs are shown in the next section). Moreover, the variations in orientation among the different crystal 

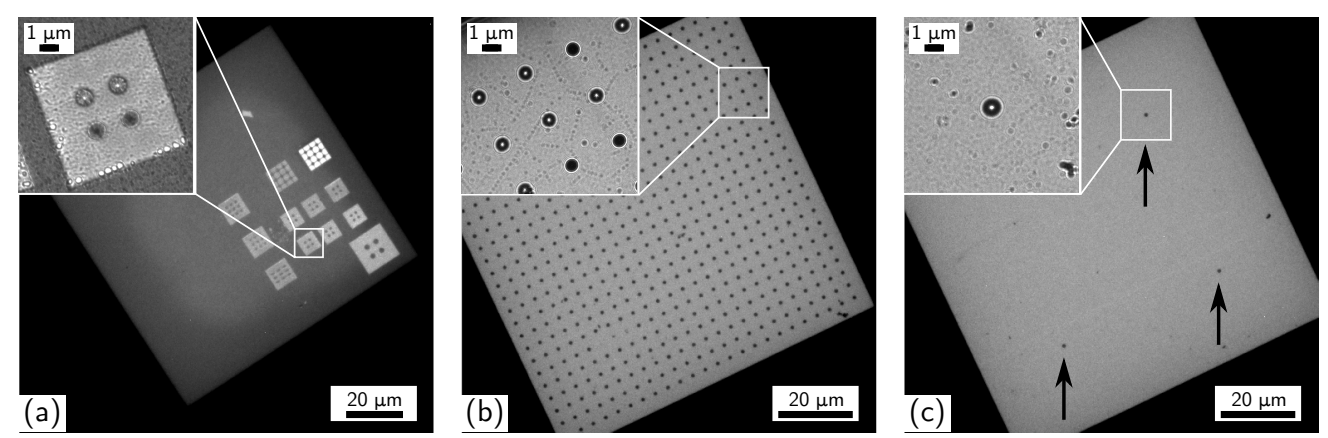

Fig. 3.2.: TEM images of the permalloy nanoislands prepared (a) by FIB-patterning, and $(b, c)$ by e-beam lithography. In the insets, the corresponding Lorentz images of the nanodiscs indicate the magnetic vortex state (bright or dark spot in the center of the discs).

grains may disturb the formation of the magnetic vortex itself by introducing spatial modulations on the magnetic energy terms related to magnetocrystalline anisotropy. The characterization of the samples crystallinity is given in Appendix A.

\subsubsection{Accumulated Heat Effects on Lorentz Image Contrast}

In the time-resolved experiments, the sample is under constant $800-\mathrm{nm}$, femtosecond pulsed laser illumination. Accordingly, the energy input occurs in a very short timescale, whereas energy dissipation takes place essentially between consecutive pulses. For instance, from the 60-nJ energy per pulse impinging on the sample, only about $6 \mathrm{pJ}$ are absorbed by the $1-\mu \mathrm{m}$ permalloy disc in $50 \mathrm{fs}{ }^{1}$, increasing its temperature by about $268 \mathrm{~K}$ at a ps timescale ${ }^{2}$. The substrate is mostly transparent to the laser beam, behaving as a heat sink for the absorptive permalloy. In the $2 \mathrm{~ms}$ until the arrival of the next pump pulse, the permalloy tends to thermalize with the substrate. The balance between heating rate (determined by the pulse energy and duration, and by the permalloy absorbance), and cooling rate (determined by pulses separation in time, and the thermal coupling between permalloy and substrate) yields an accumulated heat of the sample, determining the between-pulses equilibrium temperature of the disc/substrate achieved after a few pulses.

\footnotetext{
${ }^{1}$ We assume here the experimental conditions used in Chapter 5 (pump laser fluence of $8.5 \mathrm{~mJ} / \mathrm{cm}^{2}$ at $500-\mathrm{kHz}$ repetition rate, $50-\mu \mathrm{m}$ laser spot), and an absorbance of $25 \%$ at $800 \mathrm{~nm}$ for $20-\mathrm{nm}$ thick permalloy.

${ }^{2}$ Calculated using the material constants of Nickel, which constitutes about $80 \%$ of permalloy. Heat capacity $c_{p}=0.445 \mathrm{~J} / \mathrm{g} . \mathrm{K}$, density $\rho=8.90 \mathrm{~g} / \mathrm{cm}^{3}[116]$.
} 
The sample design affects both heating and cooling rates, but special care must be taken with the dissipation at the high repetition rates that we employ here. There are several ways that could improve heat dissipation from the permalloy disc. the most effective measure likely being the use of a conductive material, either as an additional thin layer or by replacing the amorphous substrate by a crystalline one. This approach would, however, deteriorate the magnetic contrast by increasing the overall thickness in the first case, or by introducing additional phase contrast features in the second case. Another way for dissipation improvement is to decrease the absorbed energy by removing absorbing material (i.e. permalloy) from around the disc. For this reason, we expect the lithographically prepared samples to present enhanced performance for the time-resolved experiments.

We characterize the effects of accumulated heat in the prepared samples by acquiring conventional Lorentz micrographs during laser illumination with varying pump fluence, for excitation conditions to be used in the time-resolved experiments (800-nm wavelength / 50-fs laser pulses, at 250 or $500 \mathrm{kHz}$ repetition rate). For increasing equilibrium temperature, permalloy gets partially demagnetized, altering the strength of the electron beam lensing effect by the curling magnetic vortex, and hence the phase contrast (see Equation 2.1). Figure 3.3a (left) shows a sequence of micrographs of an isolated disc for increasing pump fluence. The intensity of the bright spot at the center of the disc is affected by the laser fluence, which can be clearly identified in the azimuthally averaged intensity profiles around the disc center in Figure 3.3a (right). We now compare the different samples by tracking the intensity $I$ of the bright spot as a function of laser fluence, normalized by its value $I_{0}$ without optical excitation (Figure 3.3b). The graph evidences the improved heat dissipation as less permalloy is left around the disc of interest. The detrimental effect of higher repetition rates is shown as well.

Quantitative information on the disc temperature can be obtained by comparing the experimental fluence-dependent decrease in bright spot contrast with the theoretically predicted demagnetization of a heated magnet. In Figure 3.3d, the temperaturedependent magnetization calculated by the Weiss molecular field theory ${ }^{3}$ is compared to the experimental data. In order to renormalize the experimental data, we set the

\footnotetext{
${ }^{3}$ The reduced magnetization $m=M_{s}(T) / M_{s}(0)$ as a function of the reduced temperature $t=T / T_{C u}$ is given by $m=\tanh (m / t)$ [117]. Accordingly, $m=1$ at $0 \mathrm{~K}$ and $m=0$ at the Curie temperature $T_{C u}=850 \mathrm{~K}[118]$.
} 

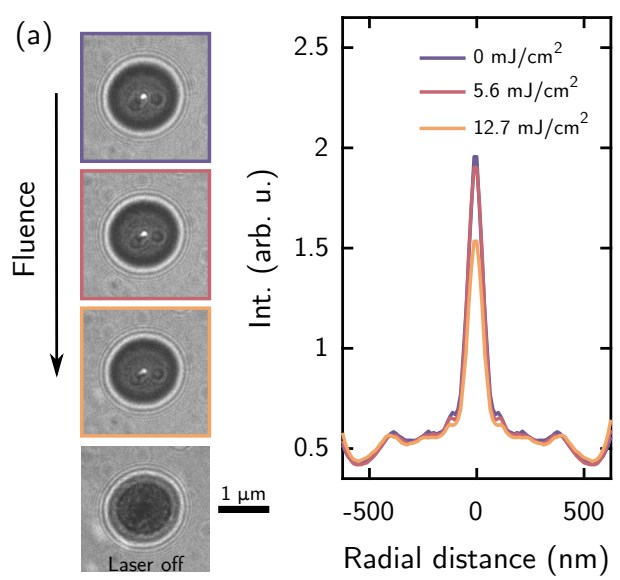

(c)

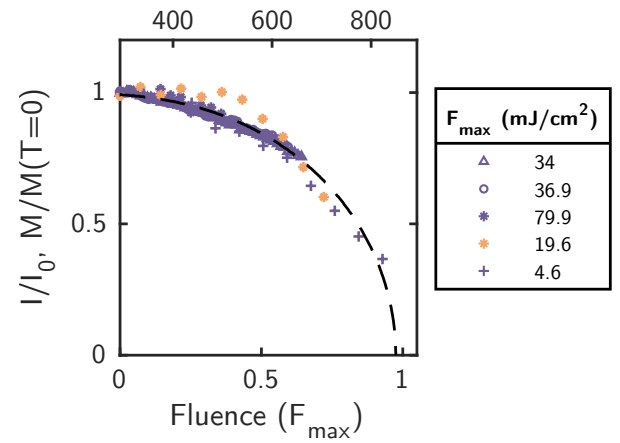

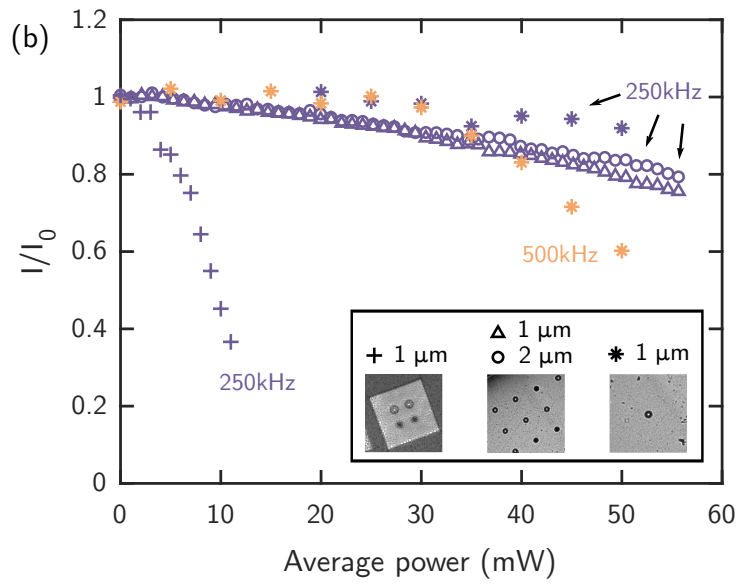

(d)

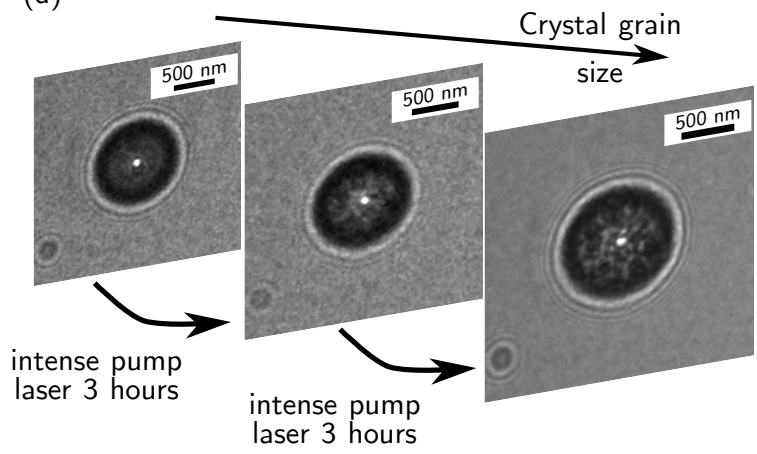

Fig. 3.3.: Thermal effects of laser illumination on time-averaged Lorentz microscopy contrast recorded using a continuous electron beam. (a) Sequence of micrographs and corresponding radial intensity profiles of an isolated $1-\mu \mathrm{m}$ disc under $500-\mathrm{kHz}$ pulsed laser illumination. The dark spot at the disc center in the last micrograph evidences the reversal of curling direction of the magnetic vortex. (b) Time-averaged effect of pump laser fluence on the contrast of the bright spot for different samples. The purple and yellow symbols correspond to pump laser repetition rate of 250 and $500 \mathrm{kHz}$, respectively. (c) Weiss molecular field theory model applied to the data in (b). The estimated pump fluence required for an equilibrium temperature of $T_{\mathrm{Cu}}$ is indicated. (d) Effect of long exposure to high fluence laser illumination. 
first and last fluence value of each data set to the corresponding predicted relative decrease in magnetization. Excellent agreement allows us to read the equilibrium temperature directly from the intensity variations, which is about $561 \mathrm{~K}$ for the isolated disc at the above mentioned experimental conditions. The comparison also suggests that the intensity of the bright spot is proportional to the magnetization, which we will discuss in more detail in the next chapter.

We now briefly discuss additional effects of laser excitation of the nanodiscs. Occasionally, the curling direction of the magnetic vortex is reversed after being exposed to high-fluence laser illumination (see the bottom micrograph in Figure 3.3a), indicating that the peak temperature of the permalloy just after the arrival of the laser pulse is above the Curie temperature. The disc is then fully demagnetized and randomly remagnetized, sometimes with the opposite curling direction. Such conditions are highly undesirable for pump-probe experiments for which a reversible phenomenon is essential. A further downside of laser illumination is the eventual irreversible crystallization of the permalloy after prolonged exposure to high equilibrium temperatures. For our time-resolved experiments, usually run over a few hours, this constitutes an important drawback of using high pump laser fluence. As shown in Figure 3.3c, the formation of larger crystal grains introduces additional contrast features, which may not only mask the contrast of the bright spot but also continuously evolve over the measurement time, compromising the time-resolved data.

Taking all the effects reported above of heat accumulation into account, we can assert that the most suitable sample system for time-resolved Lorentz microscopy of a magnetic nanostructure consists of an isolated island. Also, for the prepared magnetic discs in particular, the experiments should be performed under moderate pump fluences. In order to keep the equilibrated temperature at $600 \mathrm{~K}$, for example, the fluence must be below $10 \mathrm{~mJ} / \mathrm{cm}^{2}$ at a $500-\mathrm{KHz}$ repetition rate, or below $40 \mathrm{~mJ} / \mathrm{cm}^{2}$ at a $250-\mathrm{kHz}$ repetition rate (see Figure 3.3c). 


\subsection{Electron Pulse Properties for fs-Lorentz Microscopy}

In Section 2.2, we stressed the key role of the transverse coherence of the electron beam for phase-contrast techniques such as Lorentz miroscopy. Transverse coherence is essentially governed by the effective size of the virtual source illuminating the sample. High transverse coherence is usually achieved in TEM by inserting apertures in the condensor system, or by collimating the electron beam. In both ways, the dose (electrons/area) is significantly reduced, which is usually practicable for a conventional TEM electron gun, but can be a limiting factor for pulsed electron sources. For that reason, a characterization of the coherence properties of the electron pulses in UTEM is a requisite for performing fs-Lorentz microscopy experiments, and is the subject of this section.

\subsubsection{Performance of Photoemitted Electron Beam for fs-Lorentz Microscopy}

In order to quantify the performance of the photoemitted electron beam, it is useful to compare the capabilities of a high-brightness, continuous electron beam and photoemitted electrons for magnetic imaging. In Figure 3.4, we demonstrate the comparable quality of Lorentz micrographs obtained using the Schottky field emitter in the UTEM both in conventional operation and by laser-driven photoemission. The micrographs were obtained with exactly the same microscope parameters and gun settings, only the electron source was "replaced" by switching from one emission process to the other (see Section 2.4.2). Specifically, the condensor system was adjusted in order to conserve an astigmatism-corrected illumination with diameter of about $80 \mu \mathrm{m}$ (about $3 \mu \mathrm{rad}$ beam divergence), while the further imaging system lenses were kept fixed, providing images with a defocus of $-13 \mathrm{~mm}$.

In Table 3.1, a few parameters are listed in order to quantitatively compare the micrographs. Despite the different number of electrons and signal-to-noise ratio (SNR), the continuous electron beams obtained by both Schottky emission or cwlaser-driven photoemission perform quite similarly, and a comparable contrast is 


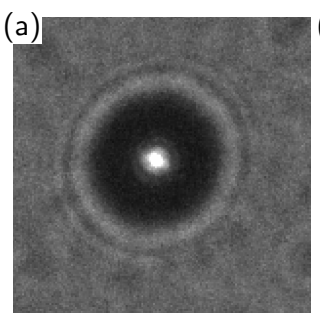

Schottky

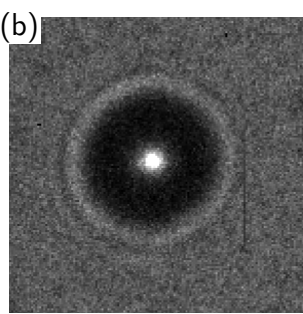

....... cw-laser

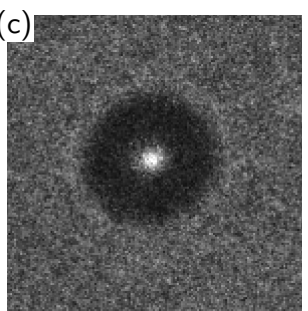

_ fs-laser

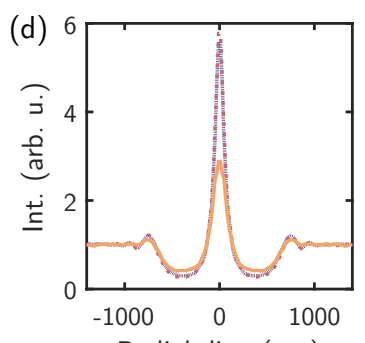

Radial dist. (nm)

Fig. 3.4.: Comparing Lorentz micrographs for (a) conventional electron beam, and photoemitted electrons obtained using (b) a cw-laser or (c) a fs-laser for exciting the electron emitter. (d) Corresponding azimuthally averaged intensity profiles about the central bright spot (color code below each micrograph).

\begin{tabular}{||l|c|c|c|c|c||}
\hline & $\begin{array}{c}\text { Exposure } \\
\text { time (s) }\end{array}$ & $\begin{array}{c}N_{e l} \\
\left(10^{5} \text { counts }\right)\end{array}$ & SNR & $\begin{array}{c}\text { Spot } \\
\text { Visibility (\%) }\end{array}$ & $\begin{array}{c}\text { Fringe } \\
\text { Visibility (\%) }\end{array}$ \\
\hline \hline Schottky & 5 & 35 & 5.5 & 89.5 & 12 \\
cw-laser & 200 & 10 & 4.4 & 90.0 & 15 \\
fs-laser & 540 & 3.5 & 3.7 & 74.0 & 5.2 \\
\hline
\end{tabular}

Tab. 3.1.: Parameters of micrographs in Figure 3.4. $N_{e l}$ is the number of electrons contributing to each image. The signal-to-noise ratio (SNR) is estimated from the mean-to-standard-deviation ratio of pixels intensity at an area of the substrate. The visibility of each contrast feature (in the radial intensity profiles) is calculated as $\frac{I-I_{0}}{I+I_{0}}$, where $I$ is the intensity of the feature. For the bright spot (first external fringe) visibility, $I_{0}$ is the intensity of the disc (the next minimum outwards).

observed for the bright spot and the Fresnel fringes ${ }^{4}$. Since these are phase-contrast features, we can assert that the UTEM photoelectron source has comparable spatial coherence properties to a thermal Schottky emitter, as quantitatively characterized in our previous works $[28,108]$. Moving on to the electron pulses generated by fs-laser driven photoemission, Figure 3.4 and Table 3.1 demonstrate the possibility of imaging the same phase-contrast features, although with a lower visibility.

The observation of phase-contrast features is an encouraging result. We need to remark the very large defocus used for the micrographs in Figure 3.4, and the high photoemission current density $\left(2.4 \mathrm{~mW}\right.$ laser power, at a $f_{\text {rep }}=250-\mathrm{kHz}$ repetition rate, yielding about 2 electrons/pulse at the sample plane ${ }^{5}$ ). Recalling Section 2.2.2

${ }^{4}$ Of course, this result is also a consequence of only looking at the radial intensity profiles to compute the visibility, since the noise level is reduced by the azimuthal averaging.

${ }^{5}$ Assuming an homogeneous electron distribution on the illumination cone, the mean number of electrons per pulse is obtained as $\frac{N_{\text {el }} A_{\text {illum }}}{A_{\text {micr. }} f_{\text {rep }}}$, where $A_{\text {illum. }}$. is the total illuminated area, and $A_{\text {micr. }}$ is the area of the micrograph shown in Figure 3.4c. The number of photoemitted electrons is, however, 100-1000 times larger close to the emitter (the transmission ratio is defined by the 

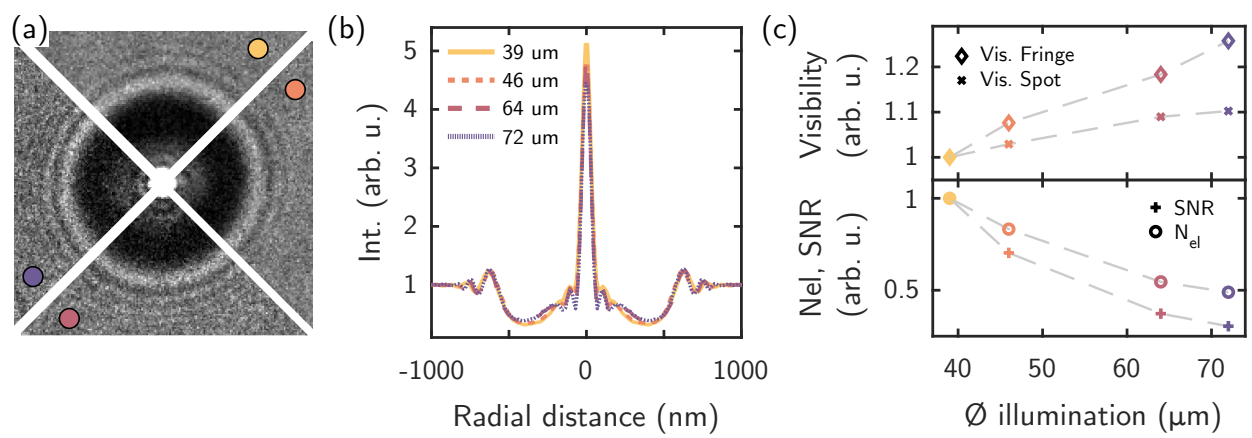

Fig. 3.5.: Effect of transverse coherence length (proportional to illuminated area diameter) on the image contrast. (a) Lorentz micrographs obtained using Schottky emission, with fixed defocus and exposure time, and with varying illuminated area diameter (circle color code, see next panels). (b) Corresponding radial intensity profiles about the central bright spot. (c) Parameters of micrographs, computed as described in the caption of Table 3.1. Each parameter is normalized according to the respective value for the $39-\mu \mathrm{m}$ diameter illuminated area.

(specially Equation 2.9), the damping in the information transfer gets more severe for higher defocus. In addition, the coherence properties of electron bunches at high photoemission current density are (moderately) worse than their continuous counterparts [108], which also reinforces the damping. As a result, it is expected that phase contrast is depreciated for the conditions used for recording the micrograph in Figure 3.4c, in comparison to a continuous electron beam emitted from the same source.

A straightforward way to control the coherence length (and consequently, the amount of phase information in phase-contrast techniques) is to spread the illumination. As stated in Section 2.3.2, the emittance is conserved by focusing and defocusing the condensor lens, and therefore a larger illumination area (i.e., a virtual source localized further away from the sample plane) leads to an increase in transverse coherence length (see Figure 2.9). The resulting effect in Lorentz imaging is illustrated in Figure 3.5, when operating the UTEM using Schottky emission. The positive effect of increasing coherence length is more pronounced in the visibility of the bright spot and Fresnel fringe (Figure 3.5c), but can also be visualized here by the improvement in the definition of further fringes both at the outer part of the disc and at the vicinity of the bright spot. Notably, the visibility of phase-contrast features is enhanced despite the lower number of electrons (Figure 3.5c).

gun operating voltages and the dimensions of limiting apertures before coupling to the microscope column). 


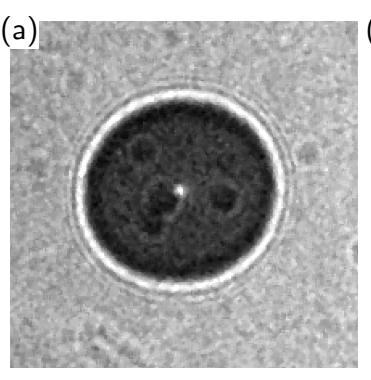

Schottky

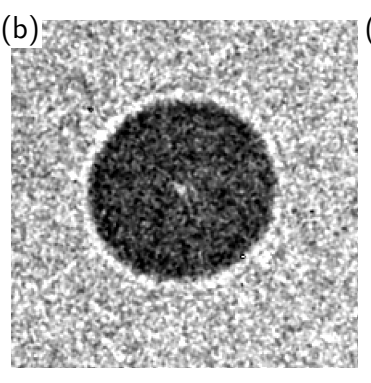

_ fs-laser

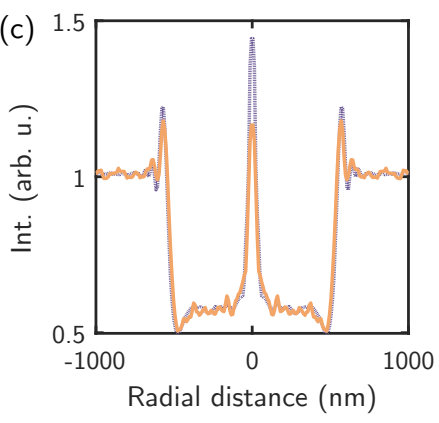

Fig. 3.6.: Comparing Lorentz micrographs for (a) conventional electron beam, and (b) photoemitted electrons obtained using a fs-laser for exciting the electron emitter. (c) Corresponding radial intensity profiles about the central bright spot (color code below each micrograph).

\begin{tabular}{||l|c|c|c|c|c||}
\hline & $\begin{array}{c}\text { Exposure } \\
\text { time (s) }\end{array}$ & $\begin{array}{c}N_{e l} \\
\left(10^{5} \text { counts }\right)\end{array}$ & SNR & $\begin{array}{c}\text { Spot } \\
\text { Visibility (\%) }\end{array}$ & $\begin{array}{c}\text { Fringe } \\
\text { Visibility (\%) }\end{array}$ \\
\hline \hline Schottky & 10 & 61 & 12.7 & 43 & 12 \\
fs-laser & 300 & 5.4 & 6.8 & 34 & 9 \\
\hline
\end{tabular}

Tab. 3.2.: Parameters of micrographs in Figure 3.6, computed as described in the caption of Table 3.1.

For the electron pulses, however, spreading the illumination at the expense of an already low signal-to-noise ratio is not sufficient. Strategies for tackling this issue will be dealt with in Section 3.3. Here, we discuss the possibility of overcoming this challenge by improving the emittance of the electron bunches. Specifically, lower emittance can be achieved by reducing the number of electrons per pulse, which is easily implemented in the UTEM by reducing the fs-laser power driving the photoemission at the microscope gun (cf. Section 2.4.2 and Ref. [108]).

A superior performance is observed for electron pulses containing up to 1 electron/pulse at the sample plane $\left(1.3 \mathrm{~mW}\right.$ laser power, at a $f_{\text {rep }}=500-\mathrm{kHz}$ repetition rate). Figure 3.6 displays micrographs obtained at fixed microscope parameters and gun settings (astigmatism-corrected illumination with diameter of about $25 \mu \mathrm{m}$ and about $7 \mu$ rad beam divergence, defocus of $-1.5 \mathrm{~mm}$ ), which are quantitatively compared in Table 3.2. For such electron pulses, the visibility of bright spot and fringes is now comparable to what is obtained using a conventional electron beam, therefore demonstrating similar transverse coherence properties. 
In summary, we demonstrate the capabilities of using the UTEM source for phasecontrast imaging with comparable quality to what is obtained with conventional TEM. Next, we present a characterization of the electron pulses used in the scope of this thesis.

\subsubsection{Electron Pulses Characterization}

A characterization of the electron pulses is essential for fs-Lorentz microscopy. Specifically, the time duration of the electron pulses is determining the time resolution of the experiments, while the transverse coherence properties limit the achievable spatial resolution. The electron pulses were characterized regarding temporal duration, energy width and transverse coherence. For the two first properties, we make use of the energy spectrometer coupled to the end of the microscope column (cf. Figure 2.12), and we demonstrate good transverse coherence properties by means of electron holography.

The electron pulses properties ultimately depend on the initial stages of the microscope electron gun setup, i.e. in the emission process and on the emitter assembly. After that, the electrons are sufficiently far apart to not experience any more spacecharge effects [108]. For the characterization presented below, we operate the emitter assembly at two different gun settings, i.e. combinations of extractor and focus electrodes potential: $U_{e x t}=1000 \mathrm{~V} / U_{f o c}=6000 \mathrm{~V}$ (setting A) and $U_{e x t}=380 \mathrm{~V}$ $/ U_{f o c}=5450 \mathrm{~V}$ (setting B). Due to higher fields at the first stage after emission $\left(U_{e x t}\right)$, setting A features reduced deteriorating effects to the coherence properties due to space-charge effects, and it is therefore used for the experiments reported in Chapter 5 (electron pulses duration and energy distribution are measured here). On the other hand, setting B provides a higher transmission ratio from the electron gun to the microscope column, and it is thus used for the dose-sensitive holographic measurements. The usability of the quantities extracted from the holograms for setting A is also discussed accordingly.

By using the energy spectrometer, we directly measure the energy width of the electron pulses (no sample inserted at the electron path). For the electron bunches used here, the energy width is determined by the number of electrons initially at the bunch when the emission takes place and hence by the laser power (or energy 

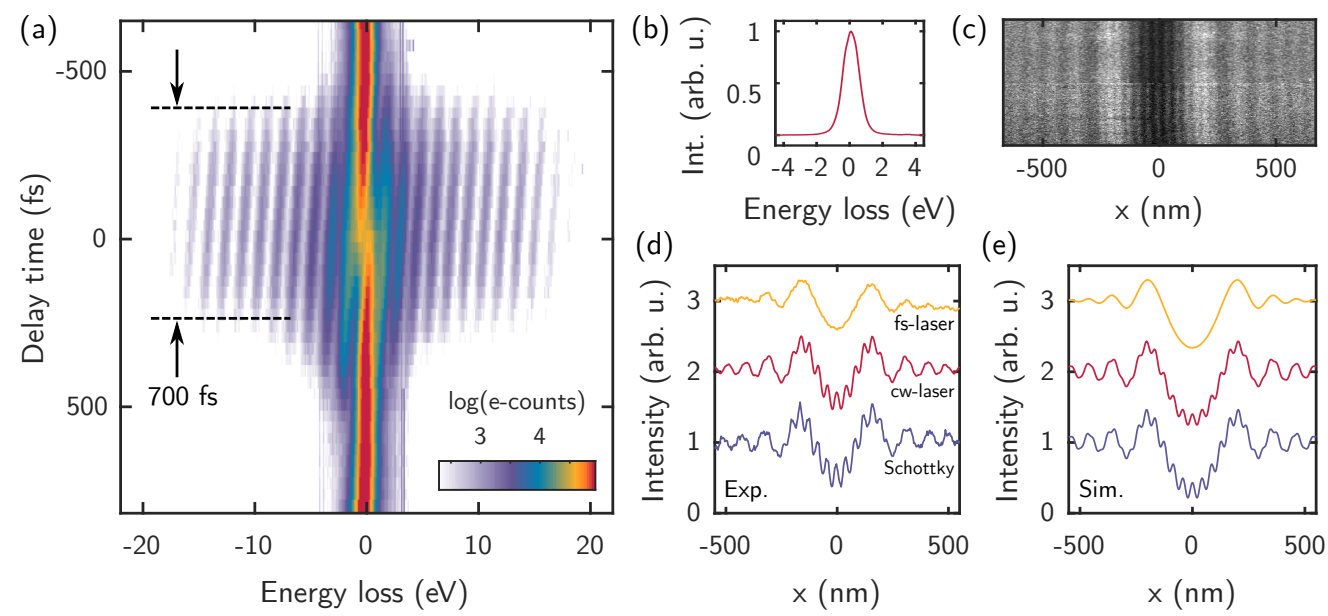

Fig. 3.7.: Electron pulse characterization. (a) Pulse duration measurement by electronphoton cross-correlation. (b) Pulse energy spectrum. (c) Empty reference holograms obtained at $9 \mathrm{~V}$ biprism voltage and fixed microscope conditions, using cw-laser driven photoemission (upper half) and Schottky emission (lower half). (d) Profiles of empty holograms, for the same parameters as in (c). (e) Simulated profiles corresponding to the ones shown in (d).

per pulse) impinging on the electron emitter. For 2.8-nJ energy laser pulses at a $500-\mathrm{kHz}$ repetition rate, typically used in the time-resolved experiments in Chapter 5 , an energy width of $1.7 \mathrm{eV}$ is obtained (full-widht at half-maximum of the energy spectrum shown in Figure 3.7b).

In order to measure the duration of the electron pulses, we make use of the electronoptical interaction ocurring at the vicinity of a material [27, 28, 105]. For very intense optical near-fields, such as the ones induced by the amplified femtosecond laser pulses used as pump pulses in the UTEM, the relativistic electrons can gain or loose energy in optical energy packets. Of course, this interaction takes place only if the electron bunches and the laser pulses overlap in position and time. The interaction is thus imprinted as sidebands on the electron energy spectrum, separated by the photon energy. The electron-photon cross-correlation can be measured by recording spectra at different delay times around the temporal overlap (Figure 3.7a). Finally, the duration of the electron pulses is extracted from the duration of the highest-order sideband. Here, we can neglect a convolution with the duration of the optical pulses, since they are much shorter (about $50 \mathrm{fs}$ ) than the electron pulses. Again, for 2.8-nJ energy laser pulses at a $500-\mathrm{kHz}$ repetition rate, a duration of about $700 \mathrm{fs}$ is obtained (see Figure 3.7a). 
The transverse coherence length is indirectly measured by recording empty reference holograms in the UTEM (Figure 3.7c), i.e. a hologram without a sample/object [119]. From the visibility of the spatial modulations in the interference patterns, information on the transverse spatial coherence properties of the illuminating electrons can be retrieved (see Section 2.2.5). Here, simulated and recorded empty holograms were compared, and the illumination angular spread $\left(\sigma_{\theta}\right)$ in the simulations was steadily incremented until the visibility matched its experimental counterpart. Figures 3.7d,e display recorded and simulated interference patterns (for Schottky emission and both continuous and pulsed photoemission, with similar microscope conditions). The modulations with a fixed, finer spacing are the holographic fringes, whereas the wider modulations at distances larger than $150 \mathrm{~nm}$ from the center are the Fresnel fringes due to the biprism edges. The simulated patterns are in good agreement with the experimental holograms. For the hologram recorded with electron pulses, only the Fresnel fringes are visible. Still, this method gives us an upper limit for the coherence length (namely, corresponding to the disappearance of holographic fringes and maintaining approximately the same relative visibility of the Fresnel fringes). From the obtained $\sigma_{\theta}$, the transverse coherence length $l_{\perp}$ is calculated using Equation 2.26, resulting in $1.468 \mu \mathrm{m}$ (Schottky), $1.261 \mu \mathrm{m}$ (cw-laser driven) and $<837 \mathrm{~nm}$ (fs-laser driven).

The coherence length is, however, strongly dependent on the chosen illumination settings (i.e., condensor lenses excitation and condensor aperture). A more relevant, conserved figure of merit can be calculated. Specifically, we can compute the degree of coherence at the sample plane, defined as $\left|\mu_{s c}\right|=l_{\perp} / d_{\text {beam }}$ (see Equation 2.27), where $d_{\text {beam }}$ is the diameter of the illuminated area. From the emittance, one can demonstrate the conservation of $\left|\mu_{s c}\right|$ independent of illumination settings (for a fixed aperture). We find, for $d_{\text {beam }}$ of about $40 \mu \mathrm{m}$ and the above $l_{\perp},\left|\mu_{s c}\right|=3.85 \%$, $\left|\mu_{s c}\right|=3.23 \%$ and $\left|\mu_{s c}\right|<1.37 \%$, respectively.

Acquiring holograms with the pulsed electron source is quite challenging. The coherent region of the electron beam $\left(l_{\perp}\right)$ has to be larger than the biprism filament, in the order of $1 \mu \mathrm{m}$ [73]. For small $\left|\mu_{s c}\right|$, spreading the illumination to achieve large enough $l_{\perp}$ strongly reduces the dose. As a result, the exposure has to be prolonged in order to acquire enough signal, which, in turn, increases the contributions of instabilities hindering the visibility of holographic fringes (see Equation 2.21 and 
comments following it). Nevertheless, the comparable $\left|\mu_{s c}\right|$ for Schottky and cwlaser driven photoemission is outstanding, demonstrating the high quality holograms obtained with photoelectrons in the UTEM (see Figure 3.7c).

Let us now discuss how the estimated degree of coherence $\left|\mu_{s c}\right|<1.37 \%$ should compare to that of the pulsed electron illumination employed in the time-resolved experiments in Chapter 5. We need to consider the following aspects:

- Gun settings: as mentioned before, operating the gun at settings B (for acquiring holograms) yields electron beams with poorer coherence properties when compared to settings A (used for experiments). For fixed illumination-limiting apertures, $\left|\mu_{s c}\right|$ may get 3-4 times larger.

- Condensor aperture(CLA) / spot size: the effective virtual source decreases for smaller CLA and/or larger spot sizes. Different combinations were used here (CLA 1/Spot 5) and in Chapter 5 (no CLA/Spot 1). The CLA affects the transverse coherence properties much more strongly than the spot size (as our reported measurements in Ref. [28]), and we estimate $\left|\mu_{s c}\right|$ to be about 2 times smaller for (no CLA/Spot 1).

- Electrons/pulse: space-charge effects depreciate the transverse coherence properties of the UTEM tip emitter [108]. For recording holograms, there are about $1.7 \mathrm{e}-/$ pulse at the sample plane. For most of the experiments in Chapter 5 , we employ lower density electron pulses.

To sum up, Table 3.3 contains the electron pulse properties for the experiments in this thesis. Electron pulse duration and energy width were measured for each condition. The values for the degree of coherence $\left|\mu_{s c}\right|$ are estimated from the values given in Ref. [108] for electron pulses of approximately the same energy width/pulse duration.

\subsection{Data Acquisition and Evaluation}

As stated previously, the transverse coherence of the illuminating electrons is an essential aspect for phase-contrast imaging. In Section 2.4.3, we discussed the limitations imposed on the coherence properties of short, few-electrons pulses, 


\begin{tabular}{||c|c|c|c|c||}
\hline $\begin{array}{c}\text { Photoemission } \\
\text { power }(\mathrm{mW})\end{array}$ & $\begin{array}{c}\text { Rep. rate } \\
(\mathrm{kHz})\end{array}$ & $\begin{array}{c}\text { Pulse } \\
\text { duration }(\mathrm{fs})\end{array}$ & $\begin{array}{c}\text { Energy } \\
\text { width }(\mathrm{eV})\end{array}$ & $\begin{array}{c}\text { Degree of spatial } \\
\text { coherence }(\%)\end{array}$ \\
\hline \hline 2.4 & 250 & 1700 & 5.1 & $<0.6$ \\
1.3 & 500 & 700 & 1.7 & $<1$ \\
0.4 & 500 & 400 & 1.3 & $<1.4$ \\
\hline
\end{tabular}

Tab. 3.3.: Electron pulse properties used for the time-resolved experiments reported in Chapter 5.

deducing that single-electron pulses have the maximum coherence length. Ideally we should then utilize single-electron pulses for imaging, which, of course, dictates the number of electrons per second $\left(\dot{N}_{e}\right)$ traveling through the column to be numerically equal to the repetition rate $f_{r e p}$ of the pulsed laser driving the photoemission. Here, we have used a maximum of $500-\mathrm{kHz}$ repetition rate for the pump-probe experiments (see limitations on $f_{\text {rep }}$ imposed by the sample accumulated heating in Section 3.1.2), which corresponds to $\dot{N}_{e}=5 \times 10^{5} \mathrm{e}^{-} /$s. As a comparison, a typical current of $1 \mathrm{nA}$ used for TEM imaging corresponds to $6.25 \times 10^{9} \mathrm{e}^{-} / \mathrm{s}$.

For fixed illumination conditions and exposure time, the dose of electrons impinging on the detector is thus $\times 10^{-4}$ smaller for the pulsed electron source we use in the UTEM, when compared to conventional TEM imaging. This means that specific strategies must be developed in order to deal with the low-signal images in fs-Lorentz microscopy. This section covers the methods established to this end within the scope of this thesis.

\subsubsection{Increasing Signal-to-Noise Ratio}

Our first concern is to boost the signal of a single image, which can be straightforwardly achieved by increasing the exposure time. This is a reasonable solution up to a few-minutes exposure, but gives rise to some drawbacks making it impracticable for longer times. Firstly, because dark counts are also linearly accumulating with exposure time and hence the background noise level is also enhanced. Secondly, because drift in the illumination or sample position during the exposure may degrade the spatial resolution. A further solution for boosting the signal is to ameliorate the dose at the sample and/or at the detector. 
In general, the dose can be improved by increasing the number of electrons per second impinging on the sample. The ways to achieve that are either by increasing the number of electrons per pulse or by increasing the repetition rate, which, as pointed above, would spoil the coherence (and temporal width) of the electron pulses or cause accumulated heating effects on the sample, respectively. A further strategy that can be adopted here is to reduce the illumination area, although this should be done in a cautious manner, as the transverse coherence length is proportional to the diameter of this area (degree of spatial coherence, see Figure 3.5). Finally, at the detector, binning of neighbor pixels can be used to raise the number of electrons (signal) per pixel, i.e., the effective recording dose.

On the other hand, one can also attempt to reduce the noise when post-processing the recorded digital images. Here, we apply two schemes for noise reduction, starting from a set of short-exposure images $(60 \mathrm{~s}$, in order to get a minimum contrast on the recorded micrograph). First, the median intensity at each pixel is calculated within the image set, thus removing noise-related outliers. Next, a spatial filter is applied to the the median image, which does have the side effect of depreciating the spatial resolution. We tested a few filters, and the ones offering the best results are compared with the unfiltered Lorentz image of an isolated Permalloy disc in Figure 3.8. The displayed histograms of an homogeneous area of the substrate indicate an improvement of the mean-to-standard-deviation ratio from 2.9 in the unfiltered image to 5 (5.5) in the filtered image using the the median (Gaussian) filter. Although performing similarly to the median filter for the image displayed in Figure 3.8, we adopt the Gaussian filter for the further experiments in this thesis, as it allows for more flexibility on the size of the area to be considered around each pixel.

\subsubsection{Drift-Correction Schemes}

A further advantage of the short-exposure approach we adopt here is that it allows for correcting slow drifts. When post-processing an image set, the shift between consecutive recorded images can be retrieved and counteracted by applying an opposing translation transformation to the individual images before combining them. Such a scheme preserves the spatial resolution achieved in an individual exposure of the set. 


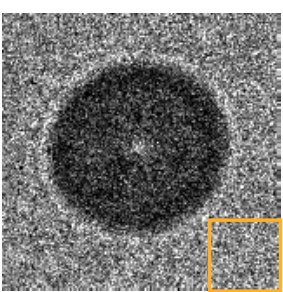

Unfitered image

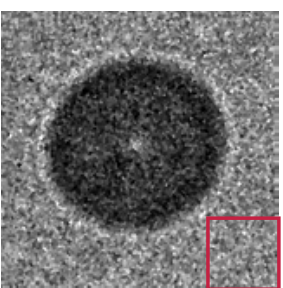

Median $(2 \times 2 \mathrm{px})$

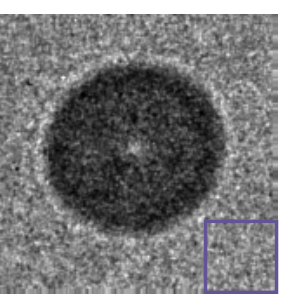

Gaussian (1.3 px)

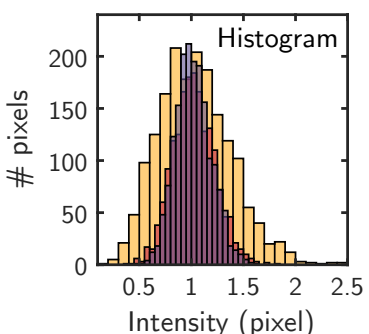

Intensity (pixel)

Fig. 3.8.: Effect of filtering a Lorentz image acquired using electron pulses. For the median filter, the pixel intensity is replaced by the median intensity of the chosen pixel area around it. For the Gaussian filter, the intensity at each pixel is replaced by the convolution of the pixel with a Gaussian function of the chosen full-width-athalf-maximum.
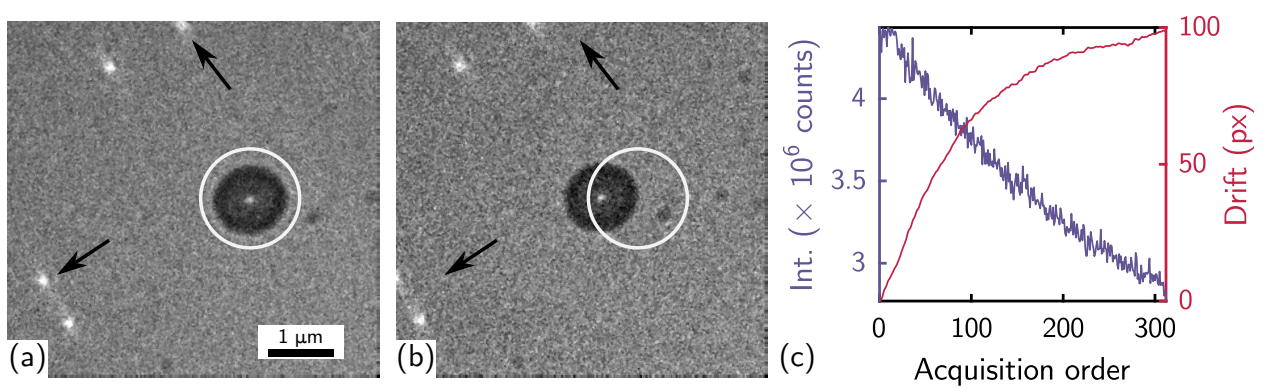

Fig. 3.9.: Drift and intensity variations along time-resolved experiments. (a,b) Median image of the (a) first and (b) last 6 images in a measurement set of 170 sequential frames. The shift can be clearly discerned from the frame displacement relative to the fixed arrows and circle. (c) Drift and intensity variations within a timeresolved experiment consisting of 312 frames.

In our experiments, drift-correction schemes undertake an additional significance. Consider, for example, a measurement sequence lasting about 1 hour for 60 delay time steps at one frame per step. As mentioned before, a set of few images must be collected for improving signal-to-noise ratio, which can be done either by repeating the whole sequence or by recording several micrographs at each delay time before moving to the next. The whole measurement can last anyway a few hours in either case. Within this time, drifts get more significant, as illustrated in Figure 3.9. In addition, the electron counts decrease over time due to the nature of the emission process (see Figure 3.9c). In order to ensure that the frames for each delay time step have comparable signal, the measurement sequence is repeated in order, and as a consequence the drift between frames corresponding to the same delay time gets relevant. 
Taking both drift and intensity variations into account, we perform data acquisition and post-processing following the general procedure described below:

1. delay-time scans with $n$ steps (1 short-exposure acquisition per step) are repeated $N$ times;

2. each image is normalized by the total image intensity;

3. find the optimized translation vector by minimizing the mean square change of pixel intensities (integrated over the whole images, relative to a reference image $)^{6}$;

4. apply the inverse translation vector to each image;

5. combine the images $I$ for each delay-time step by computing the median intensity per pixel along the $N$ repetitions (weighted median, weight $W$ given by the mean counts of a whole repetition, relative to the first one);

$$
I_{\text {delay }}=\frac{\operatorname{median}(I \cdot W)}{\sum_{i=1}^{N} W}
$$

6. apply a Gaussian spatial filter to the final images per delay, $I_{\text {delay }}$.

For step 3 of this procedure, it is important to mention the use of spatial filters to enhance the contrast between disc and substrate and hence improve the optimization algorithm convergence towards a credible solution. For the isolated disc samples, initially only a single contrast feature is available, and the algorithm is not able to find proper translation vectors for the images with lowest intensity. As a workaround, we fabricate aligning holes in the substrate, visible at the end of the arrow in Figure 3.9a. Besides, the delay-time dependent variations in the contrast of the few-pixels bright spot are largely flattened, which further allows the drift correction scheme to perform along the full sequence of images.

\footnotetext{
${ }^{6}$ We make use of intensity-based image registration functions (for instance, imregtform, and imregister) provided by the Imaging Processing Toolbox of MATLAB.
} 



\section{Numerical Analysis of fs-Lorentz}

\section{Microscopy}

CONVENTIONALLY, QUANTITATIVE INFORMATION about the magnetization distribution of a magnetic sample can be extracted from Lorentz micrographs using reconstruction approaches. In short, the interaction of the electron beam with a magnetic sample modifies the phase of the electron wavefunction, which affects the contrast in Lorentz microscopy, as discussed in Section 2.2. Inversely, information about the magnetization can be recovered from the experimental micrographs by numerically inverting the problem. Inversion methods such as the Transport-of-Intensity Equation (TIE) $[53,66,120]$ make use of discrete Fourier transformations of the micrographs, which are especially prone to showing artifacts if starting from noisy data. Due to the relatively high noise in the Lorentz micrographs obtained using ultrashort electron pulses, we have decided to use a forward approach by simulating Lorentz images for a known magnetization distribution, in order to quantitatively analyze the magnetization fields in our time-resolved data.

The simulation also allows us to evaluate further aspects of fs-Lorentz microscopy. We used a comparison between experimental and simulated results to gauge the spatial resolution achieved by ultrafast Lorentz microscopy. In addition, the simulated images illustrate the importance of coherence and dose to the achievable resolution and contrast within feasible exposure times.

This chapter covers the Lorentz image simulation and how we have used it for quantitative analysis of the time-resolved data. Section 4.1 describes the implementation of the Lorentz image simulation, introducing how we characterize the sample-electron interactions, the electron-optical parameters, and how to account for microscope aberrations, and, more importantly, for the limited coherence of the electron source. Finally, Section 4.2 summarizes the main results of the simulations, especially the high fidelity to the experimental micrographs and the calibration of the vortex disc 
magnetization from the image contrast. Parts of the results presented in this chapter were published in Ref. [109].

\subsection{Implementation of Lorentz Image Simulation}

In order to be able to simulate Lorentz images, we must first understand how the electron wave function is altered by the interaction with the magnetic sample and then establish how information is transferred in the microscope. Next, we determine the simulation parameters and how to include the electron source coherence properties in order to obtain the best agreement with our experiments.

\subsubsection{Electron-sample Interactions}

As seen in Section 2.2.2, the effects of electron-sample interactions on an illuminating electron wave function $\psi_{0}$ can be summarized in a spatially modified electron wave function $\psi(\vec{r})$ leaving the sample:

$$
\psi(\vec{r})=a(\vec{r}) e^{i \phi(\vec{r})}
$$

where $\vec{r}$ is a vector in the plane perpendicular to the electron trajectory, and the amplitude $a$ and phase $\phi$ describe the modulations imparted on $\psi_{0}$ by the scattering events. This description assumes electron-sample interactions integrated over the sample thickness.

For polycrystalline specimens, the main contribution to the amplitude modulation is the so-called mass-thickness contrast. Such contrast originates from the loss of scattered electrons down the microscope column, especially for high scattering angles. The transmitted electrons contributing to the image thus depend on the thickness-dependent scattering probability, on other material properties, and on electron energy. Therefore, significant changes in $a(\vec{r})$ can be expected to occur only between different materials or thickness steps. 
As pointed out in Section 2.2.1, the phase shift $\phi$ imparted on an electron moving through the sample at a speed $v$ is given by the Aharonov-Bohm phase shift [65]:

$$
\phi(\vec{r})=\frac{e}{\hbar}\left(\frac{1}{v} \int V(\vec{r}, z) d s-\int \vec{A}(\vec{r}, z) \cdot \mathrm{d} \vec{s}\right)
$$

where $e$ is the elementary electronic charge, $\hbar$ is the reduced Planck constant, and $V$ and $\vec{A}$ are the electrostatic and magnetic potentials inside the sample, respectively. The integrals are computed along the electron propagation direction $z$ within the sample thickness.

Let us now come to the specific sample system investigated here. The sample consists of a magnetic disc on an amorphous, electron-transparent substrate (cf. Figure 3.1 and 3.2). We assume that the amplitude and the phase modulations induced by the substrate alone are homogeneous, since there is basically no contrast feature at the substrate (both in in-focus images and in Lorentz micrographs, cf. Figure 3.2). The electrons are, however, scattered in quite a different way at the disc region. The amplitude of the exit wave function within the disc was estimated using the ratio $I_{\text {ratio }}$ between the intensity at the disc and at the substrate extracted from an in-focus image, for which phase information is irrelevant (see Equation 2.19).

The magnetic component of Equation 4.2 was calculated considering the known magnetization distribution of the vortex structure forming inside the disc of radius $R$ (Figure 4.1a). Such flux-closure configuration is established for minimizing stray fields just outside the edges of small magnets (see Section 2.1.1 and Ref. [35]); the spins align to the edge and, consequently, a curling, in-plane magnetization field develops inside the disc $[39,110]$. Although presenting a more complex behaviour close to the center of the disc (where the magnetization points out of plane and forms the vortex core), the phase shift can be closely approximated by assuming an in-plane magnetization with homogeneous magnitude. The magnetic phase shift $\phi_{m}$ thus exhibits a radially-symmetric conical shape, which, for $r<R$, is given by [67]:

$$
\phi_{m}(r)= \pm \frac{\pi B_{0} t}{\phi_{0}}(R-r),
$$

where $\phi_{0}$ is the magnetic flux quantum $\left(\phi_{0}=2070 \mathrm{~T} / \mathrm{nm}^{2}\right), t$ the disc thickness, and $B_{0}$ the in-plane magnetic induction. For $r>R, \phi_{m}$ is zero. The positive 
(a)

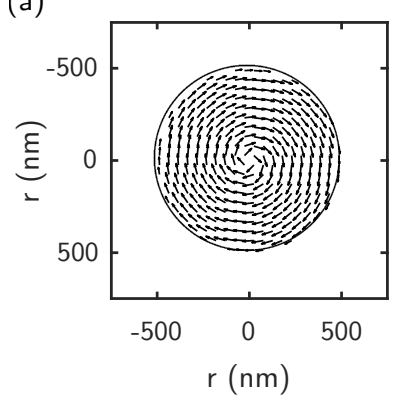

(b)

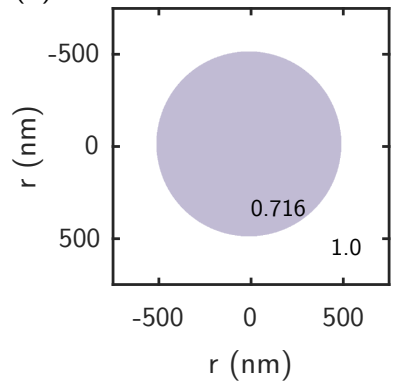

(c)

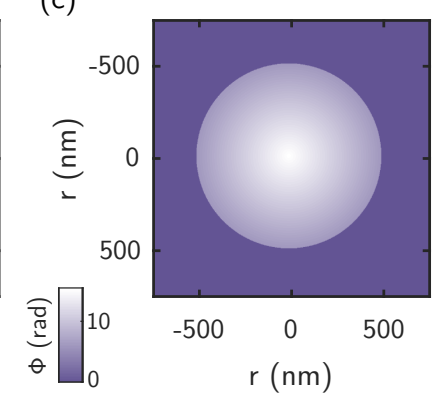

Fig. 4.1.: Magnetic disc considered for the Lorentz image simulations. (a) In-plane magnetization distribution. (b) Amplitude and (c) phase of the electron wave function after electron-sample interaction.

(negative) sign corresponds to a counter-clockwise (clockwise) curling of the sample magnetization (viewed along the $+z$ direction).

Finally, the amplitude and phase of the exit function used for the Lorentz image simulations are given by (Figure 4.1b,c):

$$
\begin{gathered}
a(r)=\sqrt{I_{\text {ratio }}} H(R-r)+H(r-R), \\
\phi(r)=\left[\frac{e}{\hbar v} V t \pm \frac{\pi B_{0} t}{\phi_{0}}(R-r)\right] H(R-r),
\end{gathered}
$$

where $H(R-r)$ is the Heaviside function.

\subsubsection{Contrast Transfer Function and Image Formation}

As described in Section 2.2.2, the image formed in a TEM can be computed from the exit wave function $\psi$ using:

$$
I=\left|\mathcal{F}^{-1}[T \mathcal{F}(\psi)]\right|^{2},
$$

where $T$ is the contrast transfer function (CTF) of the microscope, defined at the back focal plane of the objective lens (i.e., in reciprocal space). The CTF includes contributions of coherent aberrations $(\chi)$ and incoherent aberrations due to limited temporal and transverse spatial coherence ( $E^{t c}$ and $E^{s c}$, respectively). 
As mentioned in Section 2.4.2, we use the Low Magnification mode of the UTEM instrument for Lorentz microscopy. In this mode, the illumination is quite well collimated and only magnifications up to a nominal value of 6000 times are possible. Under such conditions, the relevant contributions to the CTF are related to defocus $\Delta f$ and objective lens spherical aberration $C_{s}$ (see Equation 2.4). We consider the second-order astigmatism to be corrected (which can be confirmed by the azimuthal symmetry of the Fresnel fringes immediately at the edge of the disc in Figure 3.4). Since other aberrations scale with higher order spatial frequencies $\vec{q}$ (i.e., shorter periodicity), they become relevant only for higher magnifications and are therefore neglected in our simulations.

Accordingly, the coherent aberration function used in the simulations is:

$$
\chi(\vec{q})=-\frac{2 \pi}{\lambda}\left(\frac{\Delta f}{2}(\lambda q)^{2}-\frac{C_{s}}{4}(\lambda q)^{4}\right) .
$$

We include the limited degree of temporal and spatial coherence of the UTEM electron pulses using the envelope functions $E^{t c}$ and $E^{s c}$ defined in Equations 2.5 and 2.9. In agreement with the experimental conditions, no apertures are introduced in the back-focal plane, and the complete CTF in the simulations is given by:

$$
T(\vec{q})=E^{t c}(\vec{q}) E^{s c}(\vec{q}) \mathrm{e}^{-\mathrm{i} \chi(\vec{q})} .
$$

The final image is then computed by substituting Equation 4.8 into 4.6. The image computations are performed in the commercial programming environment MATLAB. We implement a numerical calculation of Equation 4.6, and use as input (i) sample parameters to define the exit wave function according to Equations 4.4 and 4.5, and (ii) electron-optical parameters to define the CTF according to Equations 4.7 and 4.8. For all the simulations, we use a two-dimensional grid with $512 \times 512$ squared elements of $10 \mathrm{~nm}$ length (for more details, see Appendix B).

\subsubsection{Characterization of Electron-optical Parameters}

As shown in Equations 4.2, 4.7, 2.5 and 2.9, it is necessary to know a few electronoptical parameters in order to simulate Lorentz images. The electron wavelength and velocity can be directly calculated from the acceleration potential, taking into 

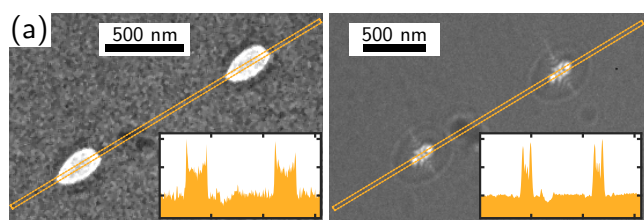

(b) 2.5

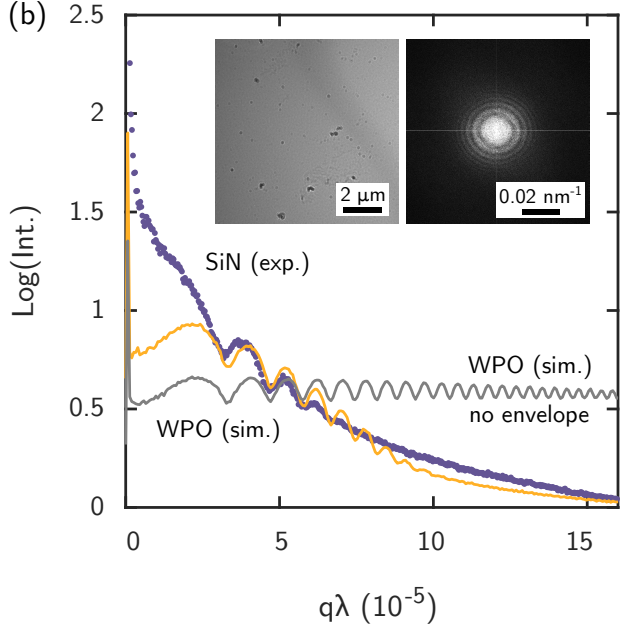

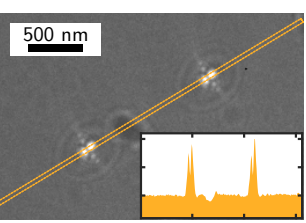
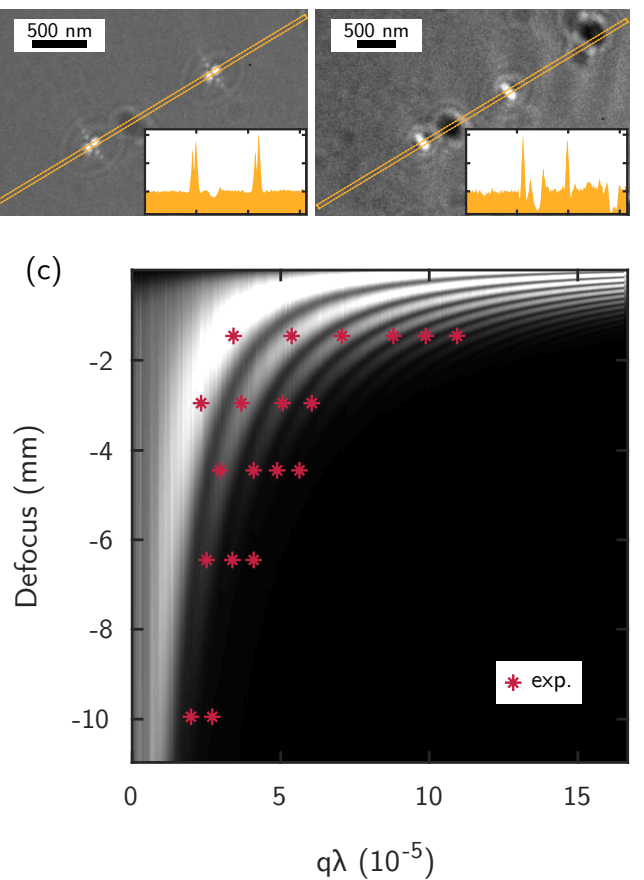

Fig. 4.2.: Characterization of microscope optical parameters. (a) Calibrating scale of defocused images from distance between reference holes $(1.3 \mu \mathrm{m})$. (b) Thon rings obtained from the diffractograms of simulated images of a weak phase object (WPO) and SiN micrograph (insets), at a fixed defocus of $-3.0 \mathrm{~mm}$. (c) Similar to $\mathrm{b}$, the simulated diffractograms and the peaks from experimental micrographs (symbols), for a defocus series.

account relativistic effects. At $120 \mathrm{kV}$ we find $\lambda \approx 3.349 \mathrm{pm}$ and $v \approx 0.587 c$. The electron energy width of the electron pulses, $\sigma_{E}=1.7 \mathrm{eV}$, was measured using an energy spectrometer coupled to the end of the microscope column (see Figure 3.7b). We take the spherical and chromatic aberrations for the objective mini-lens of a JEOL-2100F to be $C_{s}=4.61 \mathrm{~m}$ and $C_{c}=8.7 \mathrm{~cm}$, as reported in the literature [121].

The last two parameters, the defocus $\Delta f$ and the angle $\sigma_{\theta}$, were determined from the comparison between experimental micrographs and simulated images in a defocus series of an amorphous material, here the sample substrate [62, 84, 122, 123]. The method consists of computing the Fourier transform (diffractogram) of both kinds of images and finding $\Delta f$ from the position and $\sigma_{\theta}$ from the visibility of Thon rings (see insets of Figure 4.2b). ${ }^{1}$

\footnotetext{
${ }^{1}$ In the literature, the conventional method consists in evaluating the condition for $\sin \chi$ to reach a maximum and/or a zero, corresponding to the maxima and minima of a diffractogram, respectively. Defocus and spherical aberration coefficient are thus obtained by plotting the ratio $n / q^{2}$ (where $n$ is the order of each ring) versus $q^{2}$ and then fitting a straight line [123], or by plotting $n$ versus $q^{2}$ and fitting a parabola [124].
} 
The first step for the comparison is to properly calibrate the pixel size or magnification of the experimental defocused micrographs. For this, we use the spacing between fabricated holes in the membrane. Despite interference effects on the defocused micrographs of the holes, the spacing can be easily recognized (Figure 4.2a).

The amorphous, almost defect-free silicon nitride is basically electron transparent, only weakly interacting with the electrons and showing almost no contrast in an in-focus micrograph. It can thus be approximately described as a weak phase object, for which the exit wave function $\psi_{\mathrm{SiN}}(\vec{r})$ has homogeneous amplitude and small spatial phase modulations. For the simulated images, we consider that, at each pixel of the simulation grid, $\psi_{\mathrm{SiN}}(\vec{r})$ has an amplitude equal to unity and a random phase in the interval $[-\pi / 10,+\pi / 10]$. To mimic the few defects of the substrate, the phase in $20 \%$ of randomly picked pixels was replaced by either $-\pi$ or $\pi$.

Next, the azimuthally averaged diffractograms (or Fourier transforms of the images) were computed and compared with the experimental ones (see Figure 4.2b). Firstly, neglecting the coherence damping envelopes (gray curve), a first guess for the defocus was obtained by inspecting the position of the Thon rings (i.e., the diffractogram maxima). From this initial guess for the defocus and considering the influence of $E^{s c}$ and $E^{t c}$, the value of $\sigma_{\theta}$ was then continuously increased to approximately match the visibility of the Thon rings (yellow curve). Finally, the values for $\Delta f$ and $\sigma_{\theta}$ were finely adjusted to obtain a better visual agreement between the experimental and simulated Thon rings. For the experimental conditions in Section 5.1, for example, we find $\sigma_{\theta}=7 \mu \mathrm{rad}$.

We note that the damping by the coherence envelopes gets more severe for increasing defocus, and hence the number of visible Thon rings decreases. For this reason, we begin by finding $\Delta f$ and $\sigma_{\theta}$ for the least defocused micrograph. As the experimental defocus series was acquired using equally spaced objective mini-lens voltages, it is expected that the further values of $\Delta f$ in the series are spaced by a fixed difference. However, the obtained $\Delta f$ for the experimental defocus series are $-1.5 \mathrm{~mm},-3.0 \mathrm{~mm}$, $-4.5 \mathrm{~mm},-6.5 \mathrm{~mm}$, and $-10 \mathrm{~mm}$, and the positions of the respective Thon rings are shown with the complete simulated defocus series in Figure 4.2c. There are two possible reasons for the discrepancy of defocus step size for larger defocus. First, it may be related to nonlinearities of the focusing strength of electromagnetic lenses 
in a large voltage range. Secondly, an apparent shift of the Thon rings to lower spatial frequencies may occur for stronger damping, and, finally, the presence of only two or three rings on the experimental diffractograms decreases the accuracy of the method.

Finally, we discuss our method for finding and comparing the position and visibility of Thon rings by visual inspection and not by a numerical approach. Firstly, the noise in the experimental micrographs introduces artifacts in the computed Fourier transform, manifested as rapidly oscillating features in the azimuthally averaged diffractograms (see Figure 4.2b). In order to numerically find the position of the maxima, a smoothing of these curves must be performed beforehand, leading to (i) a flattening and consequently, the neglect of less pronounced peaks at higher spatial frequencies, and (ii) an unbalanced suppression of the visibility for different peaks, which may result on an overestimated value for $\sigma_{\theta}$. Secondly, in order to be able to statistically evaluate the quality of a numerical approach in finding reliable results (even after smoothening), a more complete experimental defocus series should be used.

\subsubsection{Characterization of Sample Parameters}

For the simulation of Lorentz images of the magnetic disc, the sample-related parameters in Equations 4.4 and 4.5 still need to be determined. Specifically, the products of the mean inner potential and thickness $V t$, and of the in-plane magnetic induction and thickness $B_{0} t$ are the relevant parameters, not the values of $V$ and $B_{0}$ alone.

To start, let us inspect the influence of $V$ and $B_{0}$ on the simulated images. Figure 4.3 displays intensity profiles of the disc obtained by azimuthally averaging the Lorentz images around the disc center for a $-1.5-\mathrm{mm}$ defocus and thickness $t=20 \mathrm{~nm}$ (see Section 3.1.1). For a fixed product $B_{0} t$, only the fringes close to the edge of the disc show a strong dependence on the value of $V$ (Figure 4.3a). From Equation 4.5, we see that the mean inner potential introduces a constant phase shift relative to the substrate only in the disc area. It is hence expected that, for small defocus, interference-related contrast features on defocused images are affected by changes in $V$ only in regions close to the step introduced by $V$ in the phase of the exit wave 

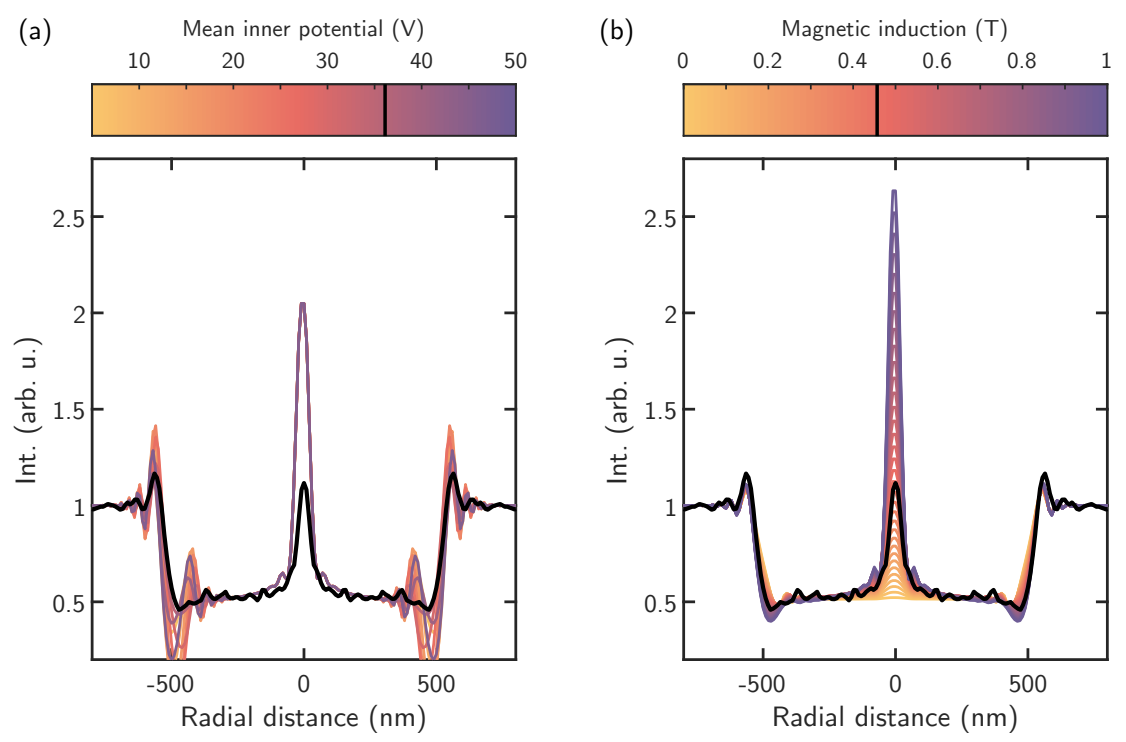

Fig. 4.3.: Azimuthally averaged intensity profiles from simulated Lorentz images at $-1.5 \mathrm{~mm}$ defocus for (a) several values of mean inner potential, and (b) several values of magnetic induction, when compared to a profile from a Lorentz micrograph (black curves). The simulation values providing the best correspondence to experiments are marked in the color scales.

function. Comparing the visibility of the inner fringes (basically absent) and of the first outer fringe to the experimental data for the same defocus (black line), we obtain $V=36 \mathrm{~V}$.

Assuming now a fixed product $V t=36 \mathrm{~V} \cdot 20 \mathrm{~nm}=720 \mathrm{~V} \cdot \mathrm{nm}$, mainly the contrast features within the disc are affected by the magnetic induction $B_{0}$, in particular at the bright spot in the center of the disc (Figure 4.3b). In fact, as discussed in Section 2.2.4 (cf. Equation 2.19), the magnetic induction is expected to affect the contrast of defocused images more strongly where the gradient of the phase changes faster, which occurs here at the vortex core (Equation 4.3 and Figure 4.1c). Comparing again with the experimental profile (black line), we obtain $B_{0}=0.46 \mathrm{~T}$.

Let us now compare the estimated values for $V$ and $B_{0}$ with the expected values from the literature. For permalloy, a mean inner potential of $26 \pm 3 \mathrm{~V}$ has been obtained from holographic experiments in the literature [125] and a magnetic induction of $1 \mathrm{~T}$ is calculated from the saturation magnetization of permalloy ${ }^{2}$. As previously mentioned, the products $V t$ and $B_{0} t$ are the relevant parameters for simulation purposes, and, as a result, an underestimated or overestimated thickness may give

\footnotetext{
${ }^{2}$ For permalloy, $M_{\mathrm{s}}=7.96 \times 10^{5} \mathrm{~A} / \mathrm{m}$ at room temperature, corresponding to a magnetic induction of $B_{0}=\mu_{0} M_{\mathrm{s}}=1 \mathrm{~T}$ (in SI units; with $\mu_{0}$ being the magnetic permeability of vacuum.)
} 
rise to the mismatch. Moreover, there may be differences in the composition of the sample used in this work from the permalloy reported in the literature. For the mean inner potential $V$ in particular, a more realistic model of the edge, with less abrupt changes on the material thickness at the boundary of the disc, could lead to a weaker influence on the fringe visibility. Of course, such a model would only be justified if the disc thickness profile was measured. In addition, for the magnetic induction, the actual thickness of the magnetic material may be smaller than the disc thickness if an oxide layer is formed (due to, e.g., sample storage conditions or aging).

To sum up, we find the values $V t=720 \mathrm{~V} \cdot \mathrm{nm}$ and $B_{0} t=9.20 \mathrm{~T} \cdot \mathrm{nm}$ from the analysis above. We note, however, that the simulated images for larger defocus, using the approach described so far, are not in good agreement with the Lorentz micrographs. In the following, we describe an improved approach, for which a readjusted value of $B_{0} t=12.24 \mathrm{~T} \cdot \mathrm{nm}$ was found to match very well the intensity of the bright spot within a wider defocus range.

\subsubsection{Accounting for Limited-coherence Sources}

Before moving to the resulting simulated images, we need to look back into the mathematical derivation of the spatial coherence envelope $E^{s c}$ of Equation 4.8 given in Section 2.2.2. At that point, a Taylor expansion up to the first order was used as an approximation for the coherent aberrations function for slightly tilted illumination, $\chi(\vec{q}+\vec{Q})$.

Figures 4.4a,b display the contrast transfer function with and without the coherence envelopes (see Equation 4.8), for two values of defocus. One can observe that the oscillations in $\chi$ get faster for increasing defocus, especially for the defocus of $-4.5 \mathrm{~mm}$ used for the time-resolved experiments in Section 5.1. As a result, the lowest order Taylor expansion used for obtaining the spatial coherence in Equation 2.9 is only of limited validity for larger defocus. An improvement can be achieved analytically by retaining higher order terms in the expansion for $\chi$ (see, for instance, Ref. [84]). This is, however, still an approximation. 

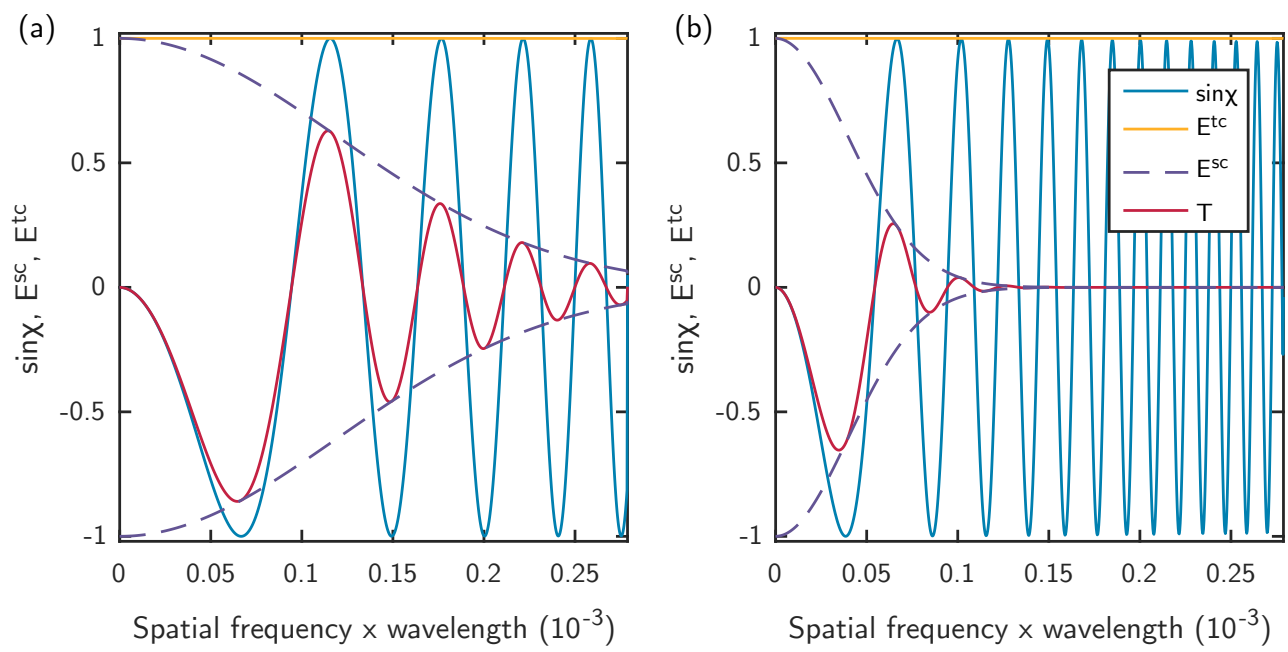

Fig. 4.4.: Contrast transfer function and the effect of the coherence envelopes for a (a) $-1.5-\mathrm{mm}$ and a (b) -4.5 - $\mathrm{mm}$ defocus.

In a more precise approach, we obtain the final simulated image intensity by numerically integrating Equation 2.6 in the following form:

$$
I(\vec{r})=\int_{i l l . c o n e} i\left(\vec{\alpha}_{i l l}\right) I_{\vec{\alpha}_{i l l}}(\vec{r}) \mathrm{d} \vec{\alpha}_{i l l}
$$

where the integration is performed for the incidence angles $\vec{\alpha}_{i l l}$ spanned by the illumination cone. Each image $I_{\vec{\alpha}_{i l l}}(\vec{r})$ is calculated using Equation 4.6 and 4.8 with $E^{s c}=1$ for a varying incidence angle $\vec{\alpha}_{i l l}$, which effectively displaces $\mathcal{F}(\psi)$ by $k \vec{\alpha}_{i l l}$ in the back focal plane. We assume the distribution of illumination directions to follow a rotationally symmetric Gaussian function with $\sigma_{\theta}$ spread (see Equation 2.8), from which we randomly sample 2000 values. The next section shows the improved agreement to experimental micrographs of the simulated images employing this approach when compared to the coherence envelope calculated using Equation 2.9.

\subsection{Results and Discussion}

\subsubsection{The Impact of Spatial Coherence}

The simulated Lorentz images obtained both by using the numerical integration approach and by employing the coherence envelope are compared with the corresponding experimental Lorentz micrographs in Figure 4.5 for two defoci. For 

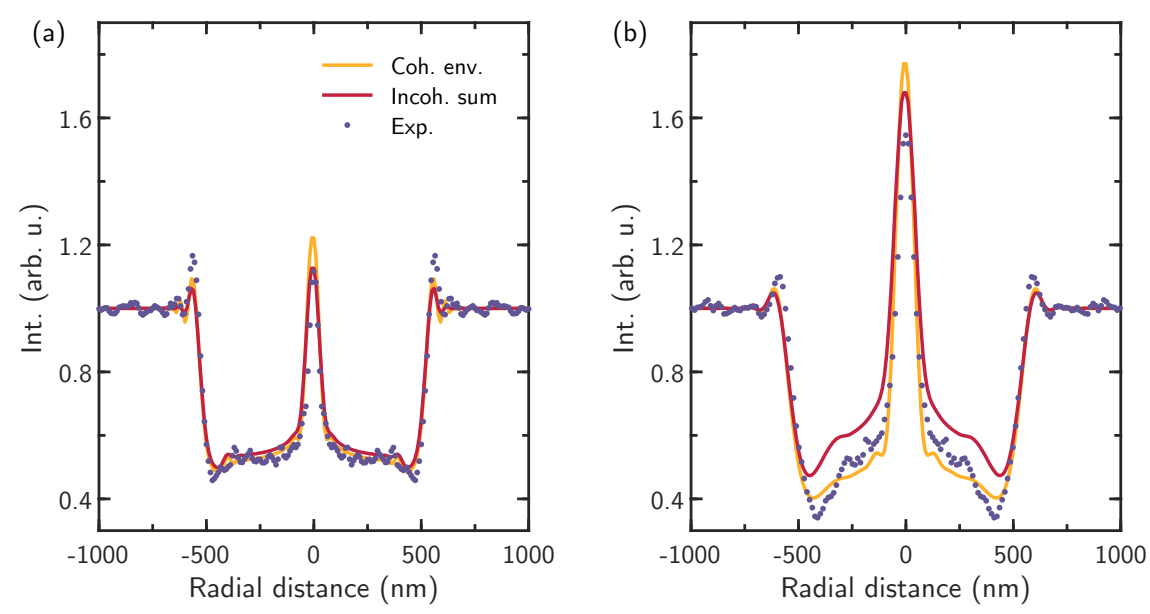

Fig. 4.5.: Comparing radial profiles of simulated Lorentz images and experimental Lorentz micrographs for a (a) -1.5-mm and a (b) - $4.5-\mathrm{mm}$ defocus.

the smaller defocus of $-1.5 \mathrm{~mm}$, both simulated profiles are quite similar to the experimental data, with minor differences in the visibility of the fringes near the disc edge and at the base of the central bright spot (Figure 4.5a). It is not feasible to judge which simulated image is closer to the experimental data, since the visibility of the features resulting from the coherence envelope approach may be hindered by the micrograph noise. On the othe hand, for the defocus of $-4.5 \mathrm{~mm}$ (Figure 4.5b) the discrepancies between the results of each approach get more severe. Not only the width of the bright spot is strongly affected by how we deal with the spatial coherence in the simulations, but also the intensity at intermediate distances from the disc center is entirely different. From the shape of the radial profiles, it is now clear that we get a superior agreement to our experiments, by numerically averaging the images obtained using different incident angles rather than by utilizing the spatial coherence envelope approximation.

Based on these observations, we can introduce two figures of merit for evaluating the quality of the simulated Lorentz images. Firstly, the full-width-at-half-maximum (FWHM) $w$ of the bright spot, which we also define as the resolution of the magnetic contrast. Secondly, the bright spot contrast $C$, defined as the relative intensity $A$ of the bright spot above the disc level $I_{\text {disc }}$, when compared to the total intensity, i.e. $C=A /\left(A+2 I_{\text {disc }}\right)$.

Let us now assess how the illumination angular spread $\sigma_{\theta}$ (i.e. the transverse coherence properties of the illuminating electrons) is affecting the resolution and 

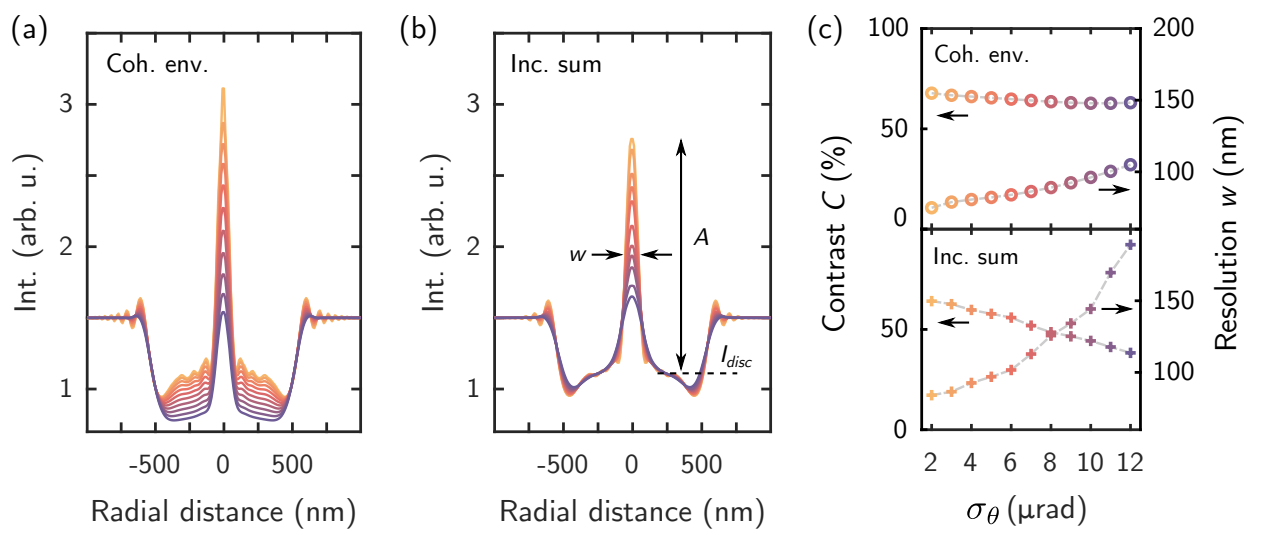

Fig. 4.6.: Comparing radial profiles of simulated Lorentz images at a $-4.5-\mathrm{mm}$ defocus and varying illumination angular spread $\sigma_{\theta}$. (a) Images obtained using the coherence envelope approach. (b) Images obtained using the averaging over $\sigma_{\theta}$. (c) Contrast $\left(A /\left(A+2 I_{\text {disc }}\right)\right)$ and resolution of the bright spot, from the profiles shown in (a,b).

contrast of Lorentz images. In Figure 4.6a,b, radial profiles of simulated Lorentz images at a fixed defocus of $-4.5 \mathrm{~mm}$ are displayed for a range of $\sigma_{\theta}$. Qualitatively, the profiles demonstrate that, as expected, the phase contrast features get more pronounced for decreasing $\sigma_{\theta}$ (i.e., for increasing transverse coherence length $l_{\perp}$ ). In a quantitative manner, Figure 4.6c shows the resolution and contrast of the bright spot in the center of the disc, as a function of $\sigma_{\theta}$. Independent of the approach for computing the simulated images, there is a general trend for improvement of both parameters for decreasing $\sigma_{\theta}$. Experimentally, a reduction in $\sigma_{\theta}$ is achieved by spreading the illumination (cf. Section 3.2.1). A comparison with the experimental profiles in Figure 3.5 also reinforces the superior performance of the numerical integration approach, since the disc intensity is virtually unaffected by variations in the illuminated area diameter.

\subsubsection{Resolution and Contrast in fs-Lorentz Microscopy}

Here, we compare the simulated Lorentz images with a defocus series of the magnetic disc obtained using electron pulses. For the images shown in this section, no pump laser was applied to the sample. The defoci are the same as those used for characterizing the electron-optical parameters in Section 4.1.3. The simulated images are in convincing agreement with the experimental micrographs (Figure 4.7a) for the utilized defocus range, which is even more evident by looking at the radial intensity profiles (Figure 4.7b). 
(a)
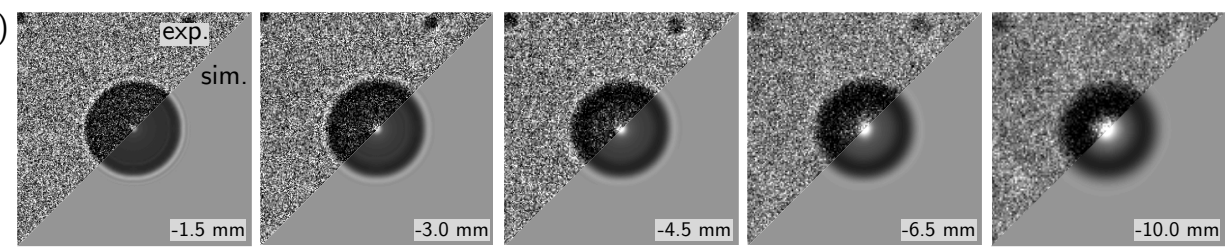

(b)

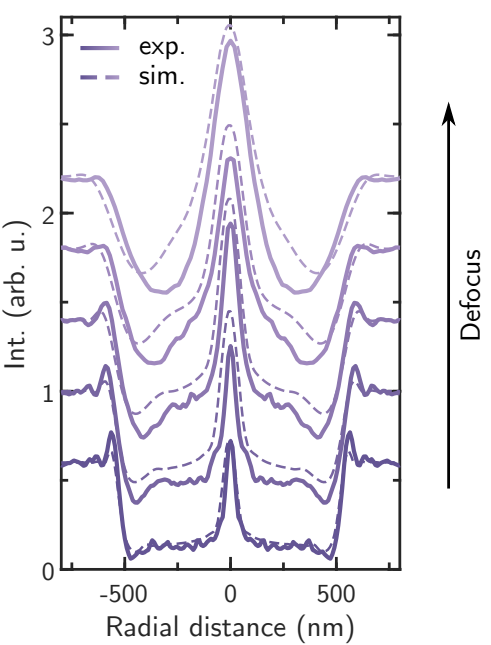

(c)

- simulation

$\diamond$ cont. e-beam

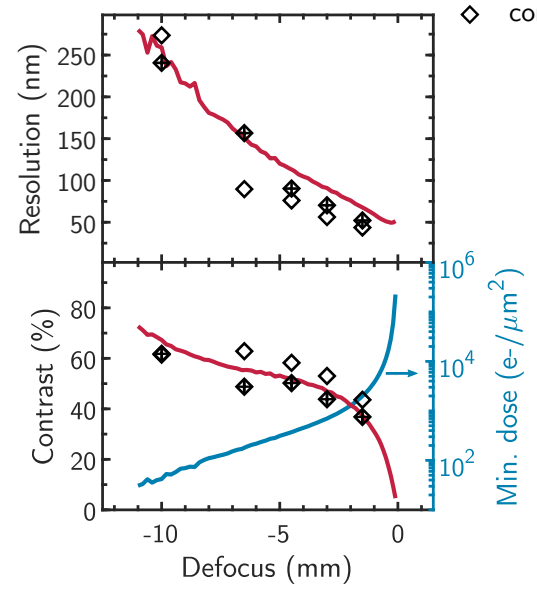

Fig. 4.7.: Comparison between the experimental micrographs and the simulated images for a defocus series of the disc. (a) Visualization of the experimental (upper half) and simulated (lower half) intensity distributions. The color scale is normalized relative to intensity at the substrate area. (b) Azimuthally averaged profiles around the disc center (vertically displaced for better visualization). (c) Resolution and contrast of the bright spot at the center of the disc. 
For both the simulated and experimental radial profiles, we extract the achievable resolution and contrast of Lorentz microscopy using electron pulses from the size $w$ and contrast $C$ of the bright spot, respectively (see Figure 4.6). Figure 4.7c shows that the parameters obtained from the simulations are in close agreement with the experimental values. Notably, for the same defoci and microscope conditions, we also find that there is a comparable resolution and contrast of the vortex core region when imaged with a continuous electron beam (Schottky emission). This result evidences that transient magnetic structures can be imaged using electron pulses with a similar quality as equilibrium structures imaged with conventional Lorentz microscopy.

Figure 4.7 also demonstrates that we can already achieve a 55-nm resolution of the bright spot at a defocus of $-1.5 \mathrm{~mm}$, which is not yet sufficient to resolve the internal dynamics of the vortex core (estimated to have a diameter of $26 \mathrm{~nm}$ [38]). In order to resolve these internal dynamics, a high electron dose is required, which we estimate in the following manner. For resolving the bright spot area of $\pi w^{2} / 4$ the minimum number of electrons must be just above the shot noise level (assumed as $1 / C^{2}$ ), corresponding to an electron dose of $\frac{1 / C^{2}}{\pi w^{2} / 4}$. The required dose steeply increases for decreasing defocus (lower panel in Figure 4.7c), which is a limitation for time-resolved experiments. For single-electron pulses at $500-\mathrm{kHz}$ repetition rate spread over an area of $30-\mu \mathrm{m}$ in diameter at the sample plane (and assuming that all electrons are detected), for example, a dose of $1 \times 10^{5}$ electrons $/ \mu \mathrm{m}^{2}$ is only achieved in exposure times as long as 2 minutes.

\subsubsection{Calibration of Transient Magnetization}

As pointed out in the beginning of this chapter, our main purpose for simulating Lorentz images is to quantitatively extract the transient magnetization from the Lorentz micrographs obtained using electron pulses. We showed a thorough characterization of all electron-optical parameters used in the time-resolved experiments in the previous sections. Now, using these parameters in the simulations, we can explore how the image contrast is affected by changes in the magnetization only.

Specifically, the defocus for the simulations was kept fixed at $-4.5 \mathrm{~mm}$ (used for the time-resolved experiments in Section 5.1). Figure 4.8b displays the series of 

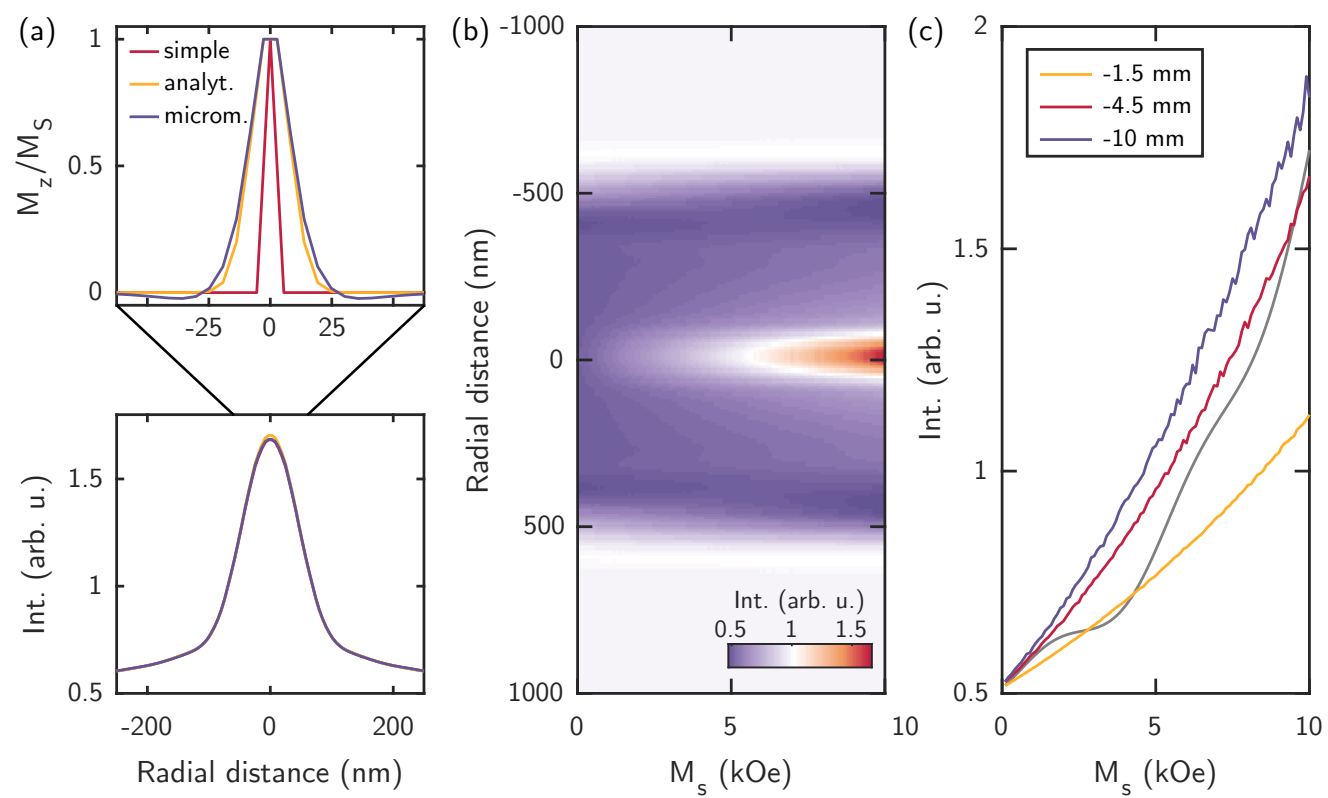

Fig. 4.8.: Calibration of sample magnetization from image contrast, for a fixed defocus of $-4.5 \mathrm{~mm}$. (a) Out-of-plane magnetization models (simple, analytical and micromagnetic) and the corresponding simulated radial profiles at a $-4.5 \mathrm{~mm}$ defocus. (b) Simulated radial profiles as a function of magnetization for the simplest model. (c) Intensity at the center of the profiles for different defoci.

simulated radial profiles for varying magnetization. The saturation magnetization $M_{\mathrm{S}}$ is calculated from the magnetic induction $B_{0}$ via $M_{\mathrm{S}}=B_{0} / \mu_{0}$, in SI units. For consistency with other references, however, we show $M_{\mathrm{s}}$ in Gaussian units. In the simulations, the thickness was fixed so that the value of $B_{0} t=12.24 \mathrm{~T} \cdot \mathrm{nm}$ corresponds to the saturation magnetization of permalloy at room temperature $\left(M_{\mathrm{s}}=7.96 \times 10^{5} \mathrm{~A} / \mathrm{m}=10 \mathrm{kOe}\right)$.

The influence of the magnetization on the radial profiles in Figure $4.8 \mathrm{~b}$ is more pronounced close to the center of the profiles. Specifically, the intensity of the bright spot strongly changes for decreasing magnetization, while its width is apparently constant. A more subtle change is observed for the contrast close to the inner edge of the disc, where a darker ring gets slimmer for decreasing magnetization. Such a stronger effect at the center than at the disc edges can be understood from the spatial modulation of the electron wave function phase by the magnetization distribution inside the disc. As seen in Section 2.2.4, the contribution of magnetization due to the magnetic vortex is stronger where the phase of the exit wave function exhibits protuberances. From the conically shaped phase depicted in Figure 4.1c, it is hence expected that the more pronounced effects occur around the center of the disc. 
For that reason, it is possible to examine how the simulated images, assuming a simplified magnetization field (cf. magnetic phase shift in Equation 4.3), compare to simulated images from more realistic field distributions. Figure 4.8a (top panel) displays the out-of-plane component of the magnetization field close to the disc center for our simplified model, for an analytical model [126] and for an equilibrium magnetic disc from micromagnetic calculations (see Section 5.1.3). Despite the differences between them, specifically on the slopes and the curvature close to the maximum, no significant changes are observed in the intensity profiles of the simulated images for a defocus of $-4.5 \mathrm{~mm}$ (lower panel of Figure 4.8a). Such an observation can be understood by recalling the results shown in Section 4.2.2, as the differences between the models lie within the vortex core and therefore are much finer than the image resolution.

The question that now arises is whether the variations of intensity at the center of the disc in Figure 4.8b should be monotonically decreasing with magnetization. Figure $4.8 \mathrm{c}$ evidences that the intensity $I$ is approximately proportional to the saturation magnetization $M_{\mathrm{s}}$ for our experimental conditions. We point out that the changes in $I$ with $M_{\mathrm{s}}$ can be more complex, even showing periodic modulations, which might complicate the quantitative evaluation of the magnetization from the image contrast. For instance, this can be seen for the gray curve in Figure 4.8c, extracted from simulated images (-4.5-mm defocus) with only the parameter $\sigma_{\theta}$ changed to $2 \mu \mathrm{rad}$. For our specific experimental conditions, however, we can use a linear function for calculating the magnetization from the amplitude $A$ of the bright spot, even for other values of defocus (Figure 4.8c). Writing the amplitude $A=I-I_{\text {disc }}$, where $I_{\text {disc }}$ is the image intensity half-way between the disc center and the disc edge, and normalizing it to be equal to unity when $M_{\mathrm{s}}=10 \mathrm{kOe}$, we find $M_{\mathrm{s}}=10 A$ (in $\mathrm{kOe}$ ).

Such a calibration is further applied in Chapter 5 , in order to obtain a measure of the transient magnetization after laser excitation from the fs-Lorentz micrographs. 



\section{Ultrafast Demagnetization Imaged by fs-Lorentz Microscopy}

IN THIS CHAPTER, we present the main results of this thesis, namely the imaging of ultrafast demagnetization in the nanoscale. In Section 5.1, we demonstrate the imaging of ultrafast demagnetization of a permalloy nanoisland with 100-nm spatial and 700-fs temporal resolution and describe the observed temporal evolution of magnetic order. Next, in Section 5.2, we show that a higher temporal resolution can also be reached, although with a noisier signal. In addition, we indicate the sensitivity of our technique to transient, optically induced near-fields. Parts of the results of this chapter were published in Ref. [109].

\subsection{Ultrafast Demagnetization of a Magnetic Disc}

\subsubsection{Specific Experimental Details}

The experiments shown in this section were conducted using the 1- $\mu \mathrm{m}$ diameter isolated permalloy disc prepared on SiN by e-beam lithography/lift-off (see Section 3.1.1 for details). The Lorentz micrographs were recorded using 700-fs electron pulses spread over an illuminated area of about $25-\mu \mathrm{m}$ diameter $\left(\sigma_{\theta}=7 \mu \mathrm{rad}\right)$ and using a defocus of $-4.5 \mathrm{~mm}$, corresponding to a coherence length of $76 \mathrm{~nm}$ (see Equation 2.7) and a spatial resolution of about $100 \mathrm{~nm}$ (see Section 4.2.2). Each image was integrated for $60 \mathrm{~s}$ (sum of 6 individual, 10-s exposures), with a total dose of about 23000 electrons $/ \mu \mathrm{m}^{2}$ and 13-nm effective pixel size.

The sample was optically excited using $800-\mathrm{nm} / 50$-fs laser pulses at a $500-\mathrm{kHz}$ repetition rate, with fluences of $3.7 \mathrm{~mJ} / \mathrm{cm}^{2}$ and $8.5 \mathrm{~mJ} / \mathrm{cm}^{2}$. The repetition rate was chosen as a compromise between acquired signal and accumulated sample heating, as discussed in Section 3.3. For each pump laser fluence, a delay time series, which consisted of 52 steps between -10 ps and 30 ps, was performed in a random sequence and repeated 6 times. Within a series, variations in electron pulse current 

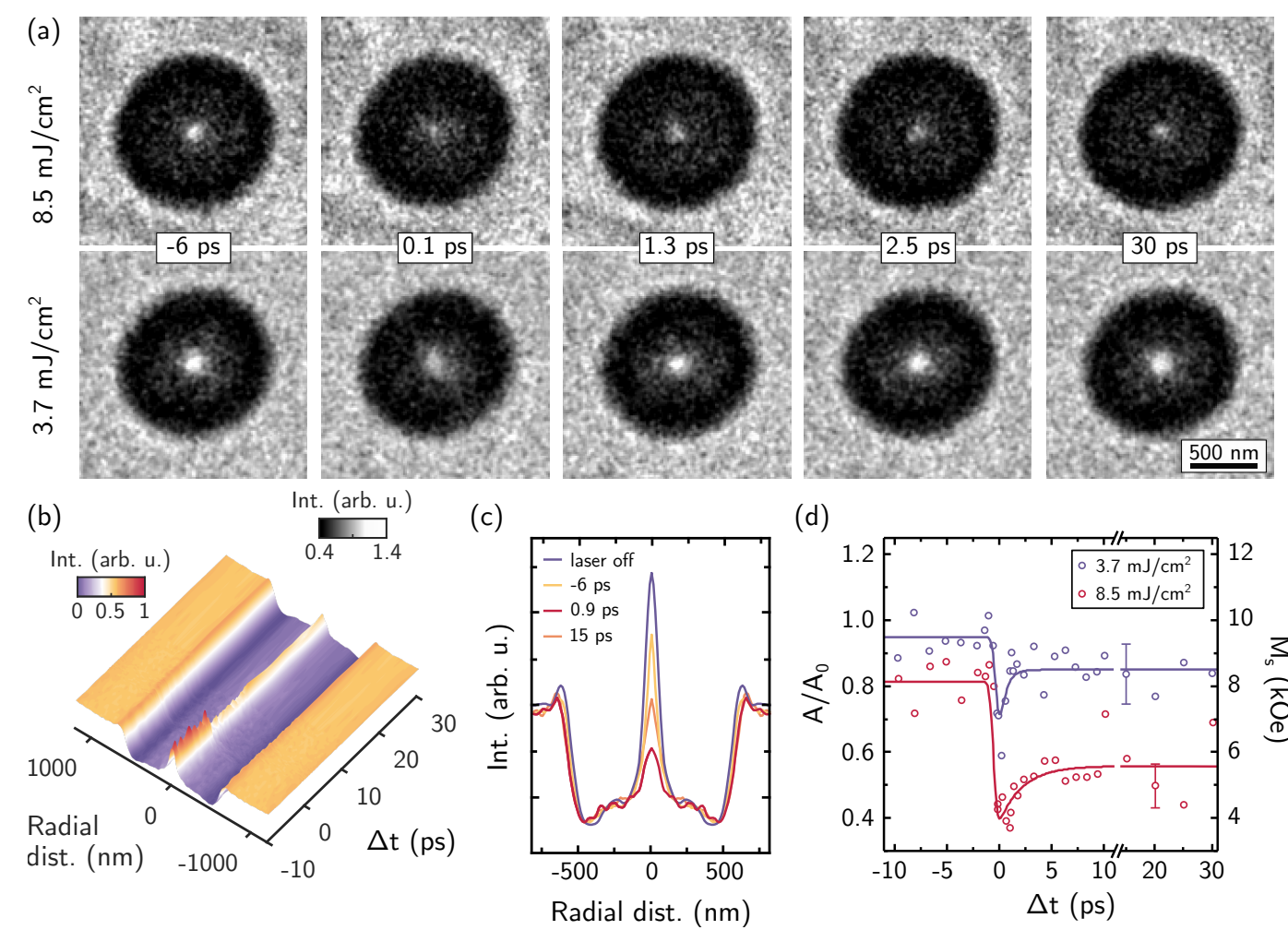

(d)

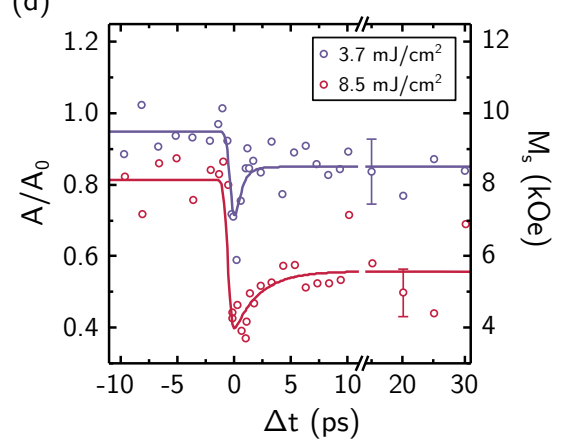

Fig. 5.1.: Temporal evolution of an optically-excited magnetic disc imaged by fs-Lorentz microscopy using 700-fs electron pulses. (a) Sequence of micrographs at indicated delay times $\Delta t$ after optical excitation, for two pump laser fluences. (b) Temporal evolution of azimuthally averaged intensity profiles for a pump fluence of $8.5 \mathrm{~mJ} / \mathrm{cm}^{2}$. (c) Intensity profiles from (b) at selected delay times, compared with a profile obtained without optical excitation. (d) Temporal evolution of the amplitude $A$ of Lorentzian functions adapted to the bright spot region of the intensity profiles, normalized by the amplitude $A_{0}$ corresponding to the micrograph obtained without optical excitation of the disc.

and slow image drift were corrected for, as described in Section 3.3.2, and a single frame for each delay time was obtained.

\subsubsection{Image Evaluation}

Figure 5.1a displays selected Lorentz micrographs of the delay time scans for both pump fluences. The image intensity is normalized to the SiN area outside of the magnetic disc. In the top row, a strong decrease in the intensity of the bright spot at the center of the disc is observed $0.1 \mathrm{ps}$ after the arrival of the pump laser pulse, and a partial recovery takes place on a picosecond timescale. For the lowest fluence (bottom row), the decrease is less severe and the recovery is both faster and more complete. 
Taking advantage of the azimuthal symmetry in the images, we azimuthally average each frame for better visualization of the dynamical evolution on the image contrast ${ }^{1}$. The full delay time series for the highest pump fluence in Figure 5.1b evidences that the most pronounced changes are localized at the disc center, as also illustrated by the radial intensity profiles for selected delay times in Figure 5.1c. For a more quantitative analysis, a Lorentzian line shape was adapted to each intensity profile by fitting the data within $250 \mathrm{~nm}$ from the center to a model function given by:

$$
I(r)=I_{0}+\frac{2 a}{\pi} \frac{w}{4\left(r-r_{0}\right)^{2}+w^{2}}
$$

where $r$ is the radial distance to the central bright spot, $I_{0}$ the offset, $a$ the area below the curve of full-width-at-half-maximum $w$ and centered at $r_{0}$. The initial parameters for the fitting were extracted from the experimental profiles, respectively, as: the base intensity level, the total intensity, the distance from the half-maximum to the central pixel and the position of the central pixel.

We can now compute the delay-dependent amplitude of the bright spot from the fitting parameters as $A=2 a /(\pi w)$. The resulting temporal evolution of $A$ is depicted in Figure 5.1d, normalized by the corresponding value $A_{0}$ obtained from the micrograph recorded with no optical excitation (see Figure 5.1c). The error bars are estimated from the standard deviation of the amplitude $A$ extracted from individual 60-s exposures during the 6 scan repetitions. The apparent behaviour observed directly in the micrographs (Figure 5.1a) follows the time evolution of $A / A_{0}$.

A further impression from the micrographs and from the radial profiles in Figure 5.1c relates to the manifestation of temporal variations only at the intensity of the bright spot, i.e., at the amplitude $A$. Now, it is interesting to examine the temporal evolution of the other fitting parameters, especially of the FWHM $w$, shown in Figure 5.2a. Here, $w$ is normalized to the corresponding value before $\Delta t=0$ to evidence only delay-dependent variations, but the error bars are calculated in an analogous manner to those corresponding to the amplitude $A$. Within the estimated errors, it is not possible to determine whether $w$ is changing with delay time. The only clear feature is a strong widening upon the arrival of the pump laser pulse, correlated with the

\footnotetext{
${ }^{1}$ As usually done in this thesis, we compute the intensity profiles from the average intensity within a ring of radius $r$ (1-pixel width).
} 


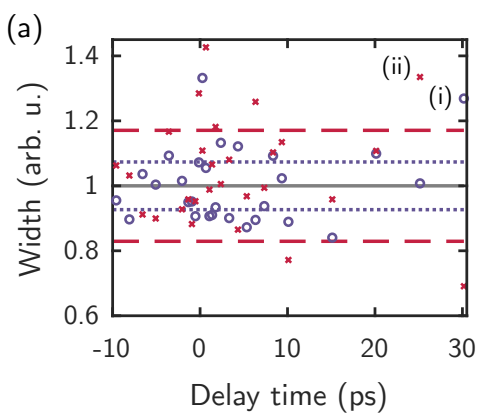

(c)
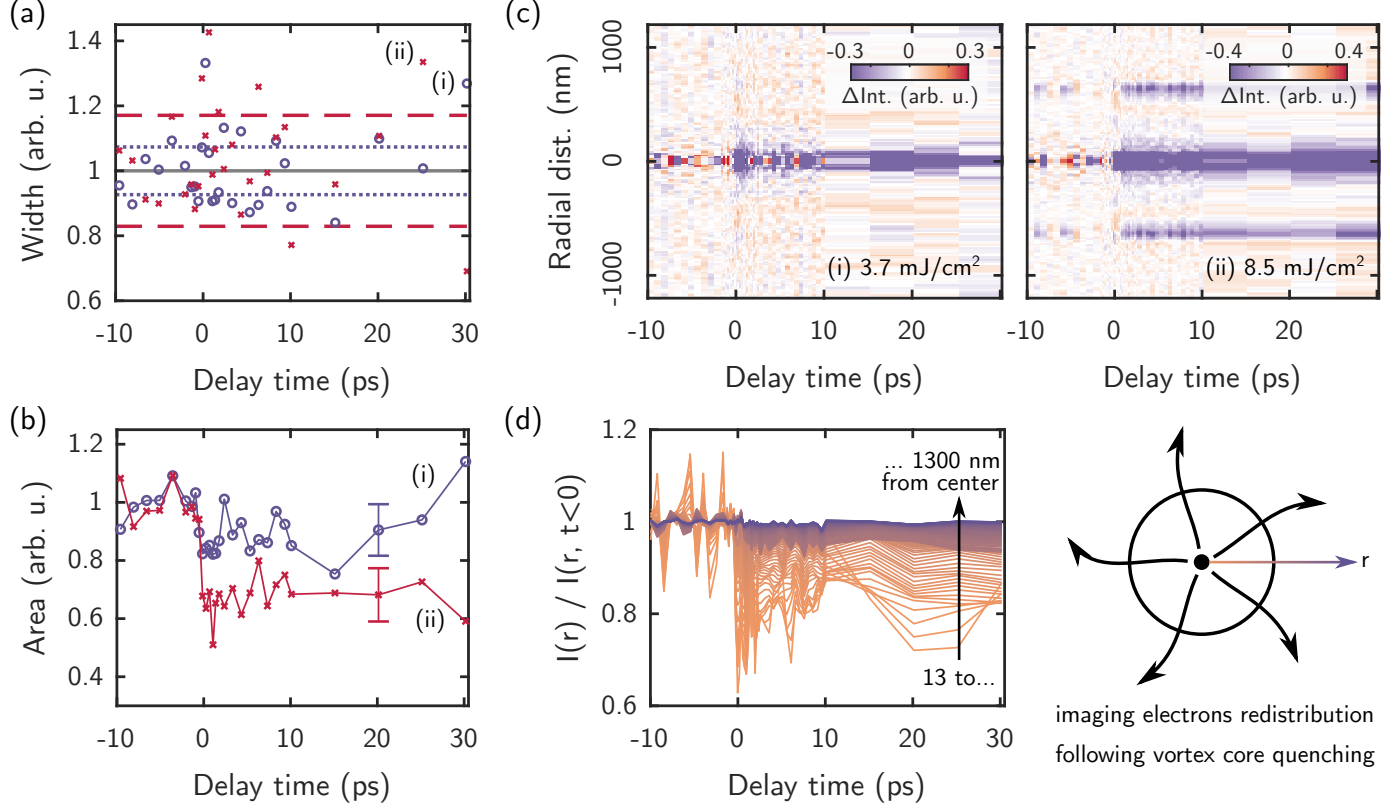

(d)

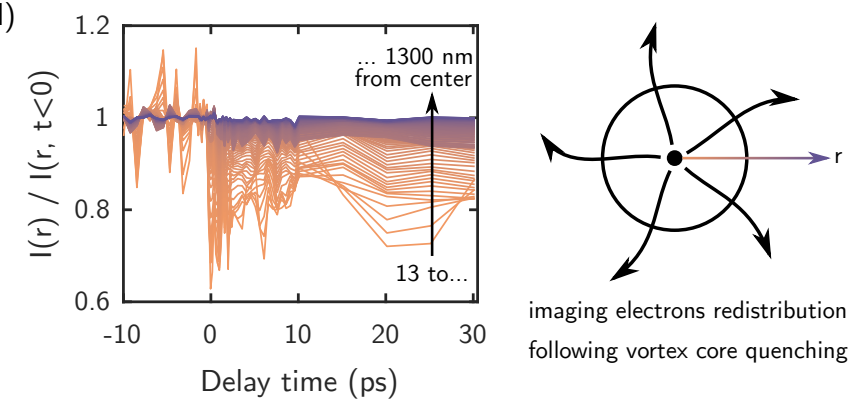

Fig. 5.2.: Imaging electrons redistribution after optical excitation of magnetic disc. (a,b) Temporal evolution of (a) width and (b) area of the bright spot at the center of the Lorentz micrographs, normalized to its corresponding values before optical excitation for pump fluences of (i) $3.7 \mathrm{~mJ} / \mathrm{cm}^{2}$, and (ii) $8.5 \mathrm{~mJ} / \mathrm{cm}^{2}$. (c) Differential azimuthally averaged intensity profiles, calculated as $\Delta I(r, \Delta t)=\frac{I(r, \Delta t)-I_{\text {mean }}}{I_{\text {mean }}}$, with $I_{\text {mean }}$ the mean profile for negative delay times. (d) Temporal evolution of the number of electrons within a circle of radius $r$, centered at the bright spot, indicates that the imaging electrons initially at the center are redistributed over a larger area.

suppression observed for $A$. In addition, the width $w$ for the highest pump fluence apparently fluctuates about a value of $15 \%$ larger than before the pump laser arrival. For further quantitative analysis, however, we disregard these observations, since the fitted model is much less reliable for less intense spots and low signal-to-noise ratio.

The different behaviours of amplitude $A$ and FWHM $w$ have a noteworthy consequence: the area $a$ under the Lorentzian model follows the temporal evolution of the intensity $A$ (Figure 5.2b). Since $a$ is related with the number of electrons contributing to the micrograph, a concomitant redistribution of the electrons must occur. In order to analyze that, Figure 5.2c displays the temporal evolution of the radial intensity profiles relative to the mean profile before $\Delta t=0$. The signal clearly decreases at the central bright spot (and at the disc rim, for the highest pump fluence), whereas a slight increase can be discerned inwards from the disc rim only about $\Delta t=0$. The profiles are, however, azimuthally averaged by normalization to the number of pixels at a distance $r$ from the center, which imply that the redistribution 
at the rim can be as significant as the one in the center. The redistribution is properly visualized in Figure 5.2d, which displays the total intensity $I(r)$ within increasingly larger circles around the bright spot, normalized by the corresponding value before time zero. At longer delay times and within $600-\mathrm{nm}$ radial distance, $I$ has $95 \%$ of its initial value, and reaches $99 \%$ for circular regions with $1-\mu \mathrm{m}$ radius.

In conclusion, by quantitatively analysing the electron redistribution within the Lorentz micrographs of the magnetic islands, we find that the variations following optical excitation are localized only at the center of the disc, and, notably, are fully described by its relative intensity (contrast). Specifically, the bright spot in this region is associated with the curling magnetization distribution of the vortex structure, which will be further examined in the next section.

\subsubsection{Temporal Evolution of Magnetization}

The evolution of the bright spot contrast observed in the Lorentz micrographs of the magnetic disc, following optical excitation, can be ascribed to laser-induced demagnetization. We can corroborate this statement by making use of the calibration for the saturation magnetization $M_{s}$ and intensity $A$ of the bright spot. The expression $M_{s}=10 A$ (in kOe), derived in Section 4.2.3, is used to track the magnetization evolution from the relative amplitude $A / A_{0}$ (see right axis of Figure 5.1d). We assume that the amplitude $A_{0}$ without optical excitation corresponds to the saturation magnetization at room temperature $\left(M_{s} \approx 10 \mathrm{kOe}\right)$. We note that, for the microscope conditions adopted here (i.e., $\sigma_{\theta}=7 \mu \mathrm{rad}$ and defocus of $-4.5 \mathrm{~mm}$ ), the simulated images are in very good agreement with the micrographs of the equilibrium structure (see Figure 4.7). Therefore, the calibration for $M_{\mathrm{S}}$ is a reliable way to track the evolution of the transient magnetization.

To the initial fast drop in magnetization (or contrast $A$ ) and slower recovery in a ps-timescale, we can adopt an exponential recovery model:

$$
A(\Delta t)=c_{0}-H(\Delta t)\left[a+b\left(1-\mathrm{e}^{-\Delta t / t_{r e c}}\right)\right],
$$

where $c_{0}$ is the (normalized) amplitude for $\Delta t<0, H(\Delta t)$ is the Heaviside step function, $a$ is the amplitude of the initial drop, $b$ is the magnitude of the partial 
recovery at later delay times and $t_{r e c}$ the recovery time constant. The model is further convoluted with a Gaussian function corresponding to the duration of the electron pulses (700 fs). The model functions, shown as solid lines in Figure 5.1d, describe well the experimental data with $t_{r e c}=0.6$ and $2.2 \mathrm{ps}$ for the lowest and highest fluence, respectively.

The temporal evolution of the magnetization and the recovery time constants are in agreement with previous ultrafast optical spectroscopy experiments [127, 128]. Using fs-Lorentz microscopy, however, we can visualize the ultrafast demagnetization of a single magnetic structure due to the laser-induced spin heating, subsequent to the arrival of the pump laser pulse, with about 100-nm spatial resolution. This constitutes an important advance for the future investigation of ultrafast behaviour of nanometric magnetic devices.

As introduced in Section 2.1.3, the ultrafast demagnetization of magnetic materials upon fs-laser excitation can be generally understood by considering individually thermalized electron, spin and lattice subsystems (three-temperature model or 3TM). The energy of the pump pulse is absorbed by the electrons and rapidly transferred to the spins within few hundreds of fs. At longer delay times, spins and electrons equilibrate with the lattice, and the recovery timescale on the order of $1 \mathrm{ps}$ is resolved in our experiments.

For delay times corresponding to a thermalized spin system (i.e., for $\Delta t<0$, and for longer delay times), we can also extract the spin temperature. As shown in Section 3.1.2 (see Figure 3.3c), a temperature can be assigned to each relative decrease in the bright spot constrast applying a Weiss molecular field theory model. Accordingly, the spin temperature rises from about $408 \mathrm{~K}(\Delta t<0 \mathrm{ps})$ to $569 \mathrm{~K}$ ( $\Delta t>5 \mathrm{ps}$ ) for the fluence of $3.7 \mathrm{~mJ} / \mathrm{cm}^{2}$, and from about $561 \mathrm{~K}(\Delta t<0 \mathrm{ps}$ ) to $753 \mathrm{~K}(\Delta t>8 \mathrm{ps})$ for the fluence of $8.5 \mathrm{~mJ} / \mathrm{cm}^{2}$. For comparison, we compute the optically induced temperature increase from the incident pump fluence and the material properties (for $\mathrm{Nickel}^{2}$, heat capacity $c_{p}=0.445 \mathrm{~J} / \mathrm{g} \cdot \mathrm{K}$ and density $\rho=8.90 \mathrm{~g} / \mathrm{cm}^{3}$ [116]). By assuming that the 20-nm thick magnetic disc absorbs $25 \%$ of the incident energy (disregarding near-field effects), we find a temperature

\footnotetext{
${ }^{2}$ Approximate composition of the permalloy discs: $80 \% \mathrm{Ni}$ and $20 \% \mathrm{Fe}$.
} 
rise of $117 \mathrm{~K}$ for the lowest fluence and $268 \mathrm{~K}$ for the highest one, in reasonable agreement with the estimate from the Lorentz image contrast.

It is also interesting to ask how the magnetization field $\vec{M}$ responds to the optical excitation. Our analysis so far assumes that only the magnitude $M_{s}$ changes with time, whereas $\vec{M} / M_{s}$ remains fixed. On the other hand, we have shown in Section 4.2.3 that any modification of the distribution of $\vec{M}$ near the vortex core is not resolvable under our experimental conditions (cf. Figure 4.8a).

To understand if any ultrafast modification on the spatial structure of the magnetization $\vec{M}$ occurs, we perform time-dependent micromagnetic simulations following ultrafast, homogeneous quenching of the magnetization in the disc ${ }^{3}$. As briefly introduced in Section 2.1.1, in micromagnetic theory the reduced field $\vec{m}=\vec{M} / M_{s}$ is calculated from energy minimization. In our model, we assume that the only time-dependent variable is $M_{s}$, whose temporal behaviour is described within the 3TM. Since changes in the spatial magnetic structure are mediated by spin waves, which propagate only over short distances on a ps-timescale, we find that there is no significant modification on $\vec{M} / M_{s}$ for $\Delta t<10$ ps.

The time-dependent micromagnetic simulation results indicate that we can trust on the extracted values for $M_{s}$ assumed by our simplistic model when calibrating $M_{s}$ (rigid $\vec{M} / M_{s}$ ). Furthermore, they evidence that the magnetic vortex responds in a non-adiabatic way to the optical excitation on a few-ps timescale, i.e., only $M_{s}$ is reduced, while $\vec{M} / M_{s}$ does not adapt to the ultrafast demagnetization.

\subsection{Varying Pump-Probe Experiment Parameters}

In this section, we evaluate Lorentz micrographs of $1-\mu \mathrm{m}$ diameter magnetic discs obtained using various experimental parameters in an analougous manner to what is described in Section 5.1.2. By comparing them, we discuss the capabilities of using fs-Lorentz microscopy for imaging magnetization dynamics with flexibility of spatial and temporal resolutions, considering specific requirements in noise level. In addition, we demonstrate the sensitivity of the technique to other transient effects.

\footnotetext{
${ }^{3}$ Micromagnetic simulations were performed in collaboration with Dr. Henning Ulrichs, using the software package mumax3 [37]. Further details are given in the Supplementary Material of Ref. [109].
} 


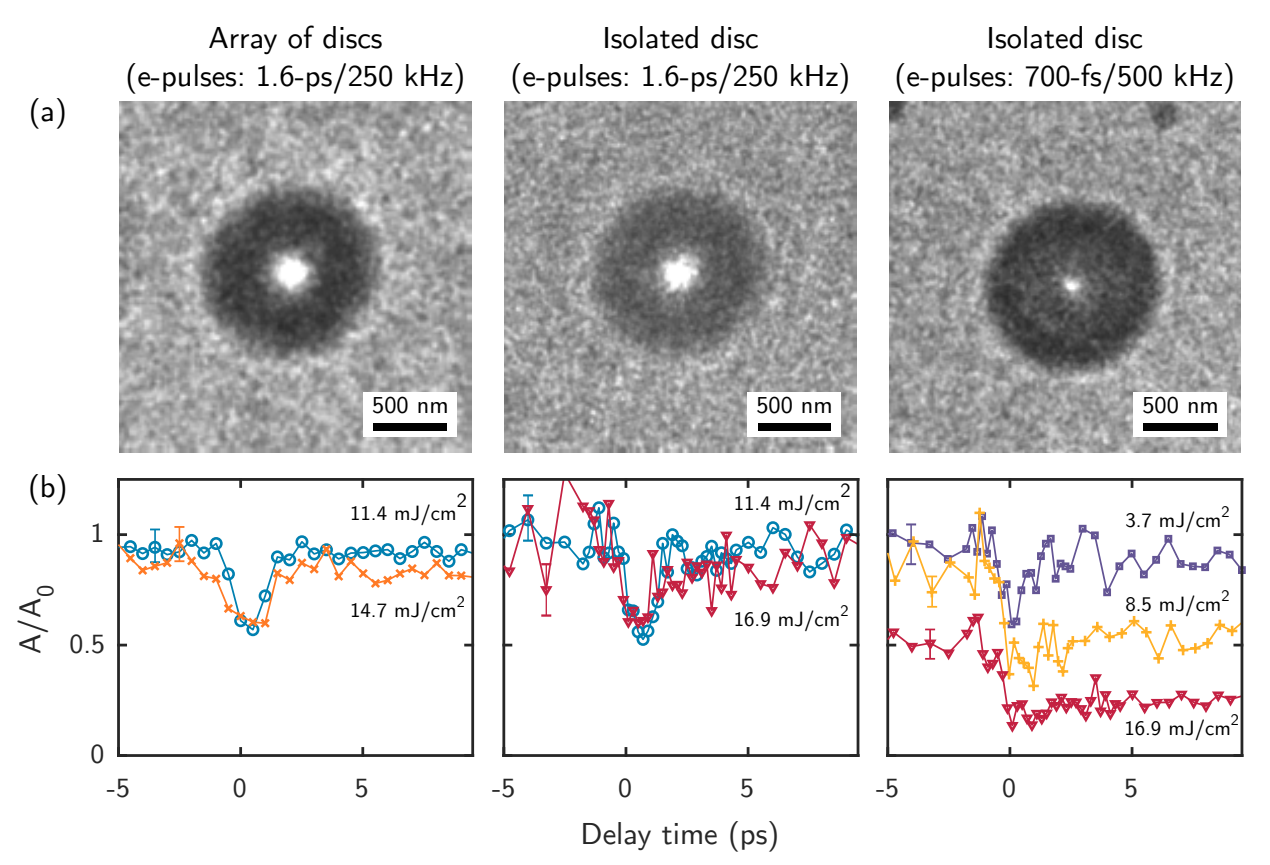

Fig. 5.3.: Time-resolved experiments using different electron pulses duration/repetition rate (label on top of each column). (a) Lorentz micrographs for negative delay times and low pump laser fluence. Defocus: $-13 \mathrm{~mm}$ (left and center), and $-4.5 \mathrm{~mm}$ (right). (b) Temporal evolution of contrast amplitude $A$ of bright spot in the center of the disc normalized to its value without optical excitation $\left(A_{0}\right)$. The fluence values are indicated, and the pump laser repetition rate is the same as the electron pulses. For the array of discs, the value of $A$ is the mean value between 6 discs within the optical excitation area.

\subsubsection{Influence on Time Traces}

Figure 5.3a displays Lorentz micrographs obtained using electron probe pulses with varying temporal duration and repetition rate. The spatial resolution varies among them due to the use of different defoci (see Figure 4.7). In spite of that, the extracted bright spot contrast ${ }^{4}$ evolves in a similar manner, for the lowest pump laser fluences (Figure 5.3b). It shows a clear dip about the optical excitation time $(\Delta t=0)$ and an almost full recovery to the initial value within a few picoseconds. There are relevant differences between these time traces, however, regarding the symmetry of the dip.

It is readily visible that the asymmetry of the dip arises only for the curve corresponding to shorter electron pulses, which indicates that the natural sample response is asymmetric and is transformed to a symmetric outline by the convolution with long electron pulses. Phenomenologically, the shape of the dip is determined by

\footnotetext{
${ }^{4}$ As usual throught this thesis, the bright spot contrast is extracted from a Loretnzian function adapted to the azimuthally averaged intensity profiles near the disc center.
} 
the electron-spin coupling (responsible for the drop) being about 10 times faster than the electron-spin-phonon coupling (responsible for the recovery), the latter occurring on a timescale on the order of $1 \mathrm{ps}$. In order to differentiate the drop from the recovery, sub-ps electron pulses are required.

In addition, the fluctuations in the time-dependent curves are strongly reduced for the leftmost data set in Figure 5.3b, since the amplitude at each delay time is averaged over a few discs organized in an array and within the area homogeneously illuminated by the pump laser. In the short time scales probed here, the presence of neighbouring nanoislands should not affect the response of a single disc, and thus the extracted amplitude develops similarly to the single-island scenario. The strong decrease in the fluctuations, however, indicates that the variations observed in the experiments with the isolated disc are still dominated by the low signal-to-noise ratio, and can be potentially reduced by a larger number of repetitions.

Lastly, it is noteworthy mentioning the effects of the optical excitation repetition rate $f_{\text {rep }}$ on the ultrafast response of the disc, for a fixed pump fluence. At $16.9 \mathrm{~mJ} / \mathrm{cm}^{2}$, for example, the response of the isolated disc (see central and rightmost plots in Figure 5.3b) is severely modified for higher $f_{r e p}$. This is associated with the higher cumulative increase in temperature for $500 \mathrm{kHz}$ when compared to $250 \mathrm{kHz}$ (see Figure 3.3), which of course has an impact on the heat capacity of the electronic system.

\subsubsection{Temporal Duration of Electron Probe Pulse}

In the Göttingen UTEM, we have demonstrated record electron pulse durations of 200 fs [28]. For reaching such short pulses, however, the electron current at the sample plane is severely reduced [108]. Especially for phase-contrast imaging as Lorentz microscopy, we have discussed the challenges of using low-current illumination in Section 3.3.1. In the present section, however, we demonstrate time-resolved Lorentz imaging using 400-fs electron pulses, for which a compromise between achievable signal-to-noise ratio and short duration of the electron pulses is still feasible.

Figure 5.4a displays the Lorentz micrographs obtained using 400-fs and 700-fs electron pulses at a $500-\mathrm{kHz}$ repetition rate, for a fixed exposure time. There is 
evidently higher noise levels when imaging with shorter electron pulses, as expected for a lower illumination current. This is observed both at the substrate region of the micrographs and on the disc intensity level in the azimuthally averaged profiles (Figure 5.4c, left). Remarkably, the spatial resolution remains almost unnaffected. In a pump-probe experiment with moderate pump fluence of $5.6 \mathrm{~mJ} / \mathrm{cm}^{2}$, we note that the bright spot can be resolved with similar quality even at the lowest contrast (for delay time of $0 \mathrm{ps}$, see Figure $5.4 \mathrm{c}$, right).

For both $400-$ fs and $700-$ fs electron pulses, the temporal evolution of the bright spot contrast is shown in Figure 5.4b. Despite the somewhat noisier micrographs, it is clear that the dip just after 0 ps is better resolved when using the shortest 400 -fs electron pulses. Similarly to Section 5.1.3, we adapt the model of Equation 5.2 to the experimental points (plotted as solid lines in Figure 5.4b), and find very similar recovery time constants for each data set: 1.3 ps and 1.2 ps by using the 400 -fs and 700-fs electron pulses, respectively.

Although still not short enough to resolve the onset of the demagnetization (occurring on timescales of about $100 \mathrm{fs}$ ), the achieved time resolution of $400 \mathrm{fs}$ allows us to image the remagnetization process with a very good combination of spatial and temporal resolution.

\subsubsection{Temporal Duration of Laser Pump Pulse}

Analogously to employing long probe pulses, the use of long pump pulses for pumpprobe experiments smoothens the observed temporal evolution. Now, however, this is not only due to convolution of sample response and probe duration, but also to the system response itself.

By stretching the optical excitation pulses in our experiments, we are able to observe an additional physical effect. In the time-resolved Lorentz micrographs for delay times within the duration of the pump pulses, an elongation of the usually round bright spot along a specific direction can be seen (Figure 5.5c). For quantitative 
(a)
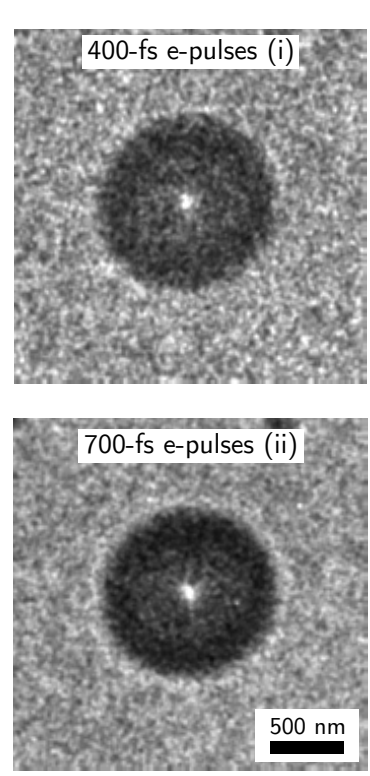

(b)
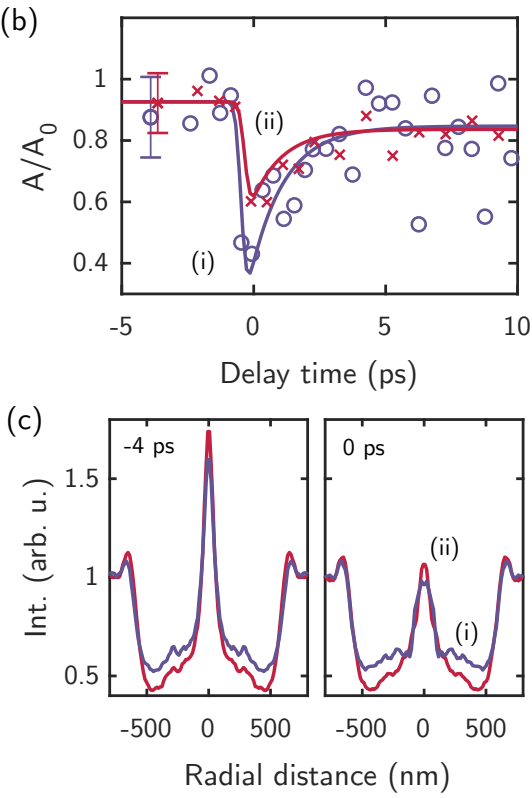

Fig. 5.4.: Time-resolved experiments using shorter electron pulses. (a-c) Pump-probe experiments at $500-\mathrm{kHz}$ repetition rate, with a pump fluence of $5.6 \mathrm{~mJ} / \mathrm{cm}^{2}$, and using (i) 400 -fs or (ii) 700 -fs electron pulses. The Lorentz micrographs (-4.5-mm defocus) before laser excitation are compared in (a), whereas (c) displays radial intensity profiles for two selected delay times. In (b), the extracted temporal evolution of contrast amplitude $A$ of the bright spot in the center of the disc normalized to its value without optical excitation $\left(A_{0}\right)$ is displayed.

evaluation of the elongated spots, we now adapt a 2-dimensional Lorentzian model function given by

$$
f(x, y)=z_{0}+\frac{a}{\left(\frac{x-x_{0}}{w_{x} / 2}\right)^{2}+\left(\frac{y-y_{0}}{w_{y} / 2}\right)^{2}}
$$

to the intensity distribution inside the magnetic disc. The function describes a peak-like surface displaced a distance $z_{0}$ above the $x y$-plane, centered at $\left(x_{0}, y_{0}\right)$, with full-width-at-half-maximum (FWHM) $w_{x}$ and $w_{y}$ along the $x$ and $y$ directions, respectively. The area of the bright spot is given by $a$.

Figure 5.5b (top) demonstrates that the area $a$, which is proportional to the total intensity of the feature, behaves in a similar way to the intensity $A$ from the experiments using relatively longer electron probe pulses (cf. leftmost panel in Figure 5.3b). The intensity is, as usual, showing the response of the transient magnetization to a long optical pump pulse, convoluted with the duration of the probe pulse. 
(a)

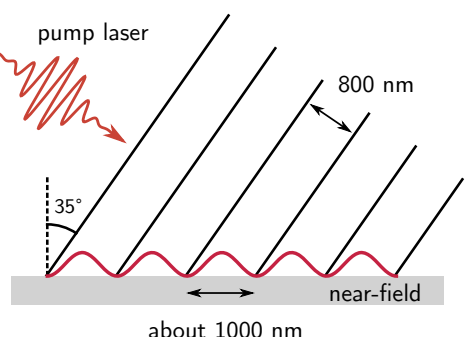

(b)

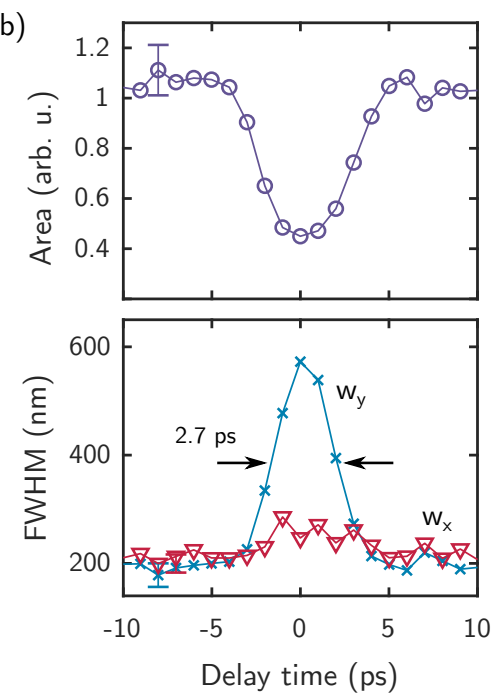

imaging electrons

deflection

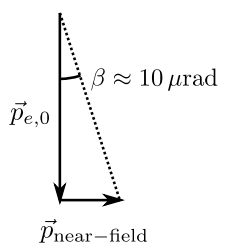

(c)

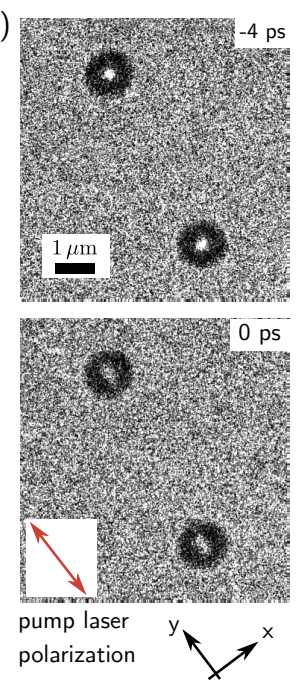

(d)
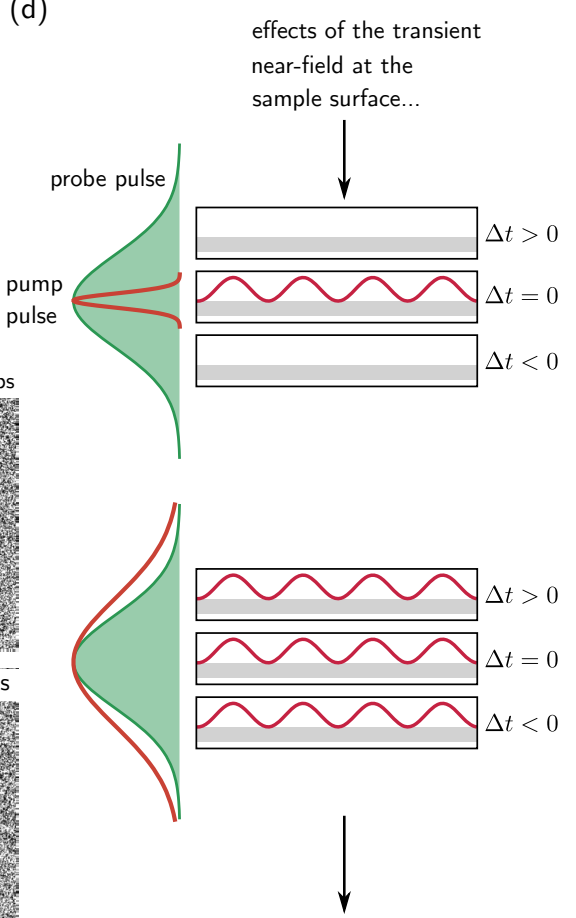

are averaged within the the duration of the probe pulses

Fig. 5.5.: Time-resolved experiments using electron-probe and laser-pump pulses of similar duration. (a-c) Pump-probe experiments at 250-kHz repetition rate using 1.6-ps electron probe pulses, and 3-ps laser pump pulses of $8.5 \mathrm{~mJ} / \mathrm{cm}^{2}$ fluence. In the classical picture sketched in (a), the probing electrons are deflected by the optically-excited near-field within the duration of the laser pulse. As a result, the bright spot in the Lorentz micrographs (-13-mm defocus) shown in (c) is stretched along the in-plane polarization component $y$ for short delay times. The observed temporal evolution of the spot area and widths in both $x$ and $y$ directions are shown in (b). (d) Physical picture of the recorded image as a superposition of transient configurations probed along the electron pulse duration. The contribution of the near-field effect to the image contrast is therefore more relevant when both pulses have similar duration (bottom). 
The FWHM displayed in the bottom panel of Figure 5.5b, however, evolves with delay time in a very contrasting way to what is observed for short pump pulses (see Figure 5.2a). The elongation in the micrographs is reproduced but, more importantly, the evolution in the indicated $x$ and $y$-directions is strongly decoupled. In the $y$-direction, the FWHM $w_{y}$ at very short delay times gets about 3 times larger than its corresponding values at longer delay times, whereas the FWHM $w_{x}$ in the $x$-direction is almost constant, varying only about $15 \%$ within the same delay time range. Interestingly, the strongest elongation and the laser pump pulse have about the same duration ( $3 \mathrm{ps}$ ), indicating that the observed response is related to a process lasting only while the laser field is present. Such observation is further corroborated by the elongation direction, which is parallel to the pump laser polarization.

The observed effect is actually associated with the electron-optical interaction due to the laser-induced near-fields. It is closely related to the observation of sidebands in the electron energy spectra used for evaluating the electron pulse duration (cf. Figure 3.7a), but now the relevant momentum exchange occurs in transverse direction [129]. Figure 5.5a schematically shows the classical physical picture behind our observations. The incident laser electric field induces a spatial modulation of the surface charges (near-field) along the direction of the in-plane component of the laser polarization (Figure 5.5a, left). As a result, the probing electrons impinging on the sample will be deflected (Figure 5.5a, right). The wavelength of the charge accumulation modulation is estimated considering the grazing incidence angle of $55^{\circ}$ to be about $1000 \mathrm{~nm}\left(800 \mathrm{~nm} / \cos 35^{\circ}\right)$. From the corresponding near-field momenta, the deflection due to a single modulation is on the order of $10 \mu \mathrm{rad}$, which originates a streak-like contrast feature of about $130 \mathrm{~nm}$ length in a $-13-\mathrm{mm}$ defocus Lorentz micrograph. The observed elongation to about $600 \mathrm{~nm}$ (Figure 5.5b, bottom) indicates the participation of 4 to 5 photons in the process.

We note that the effect is not connected to the length of the pump pulses alone, but with the comparable duration of laser pump and electron probe pulses. As shown in Figure 5.5d, the image formed by an electron pulse represents a temporal average of the transient configurations that the sample experiences within the duration of the probe pulse. As a result, the contribution of effects accompanying the pump pulse to the final image, such as the near-fields at the sample surface, is reduced if the probe 
pulses are much longer than the pump pulses. That explains why the effect is not perceptible in the experiments shown in the preceding sections.

As a final remark, this observation establishes fs-Lorentz microscopy as a technique able to image transient electromagnetic fields in general with high spatiotemporal resolution. 


\section{Conclusion}

\subsection{Summary}

In this thesis, we present the development of fs-Lorentz microscopy, a phase-contrast technique in Ultrafast Transmission Electron Microscopy (UTEM) sensitive to the magnetic induction in a sample. We apply this technique to map the optically induced ultrafast demagnetization in a single nanostructure. In this section, we summarize our main findings.

Regarding the development of fs-Lorentz microscopy:

- We demonstrate that fs-Lorentz microscopy enables imaging of magnetic structures with spatial resolution near to that of conventional, continuous electron beams, provided a high-coherence electron source as the one employed in the Göttingen UTEM instrument. In addition, high temporal resolution is obtained.

- We investigate (numerically and experimentally) the possibilities and difficulties for enhancing spatiotemporal resolution with sufficient image contrast.

- We establish ultrafast Lorentz microscopy with spatiotemporal resolutions of $55-\mathrm{nm} / 700$-fs or $100-\mathrm{nm} / 400$-fs.

- Enhancing the signal-to-noise ratio by increasing the laser repetition rate must be carefully considered, since accumulated heat effects play an important role due to the low dimensionality of the sample systems in TEM.

- We show the correspondence between the fs-Lorentz image contrast and the transient magnetization field (for a magnetic vortex), enabling to quantitative analysis of the latter.

The unique capabilities of fs-Lorentz microscopy, especially the high spatiotemporal resolution and the direct sensitivitiy to the transient magnetic induction, were 
demonstrated by visualizing ultrafast demagnetization in a single magnetic permalloy island:

- We track the temporal evolution of the magnetization following optical excitation in a single, isolated $1-\mu \mathrm{m}$ magnetic disc in the vortex state.

- The optically induced ultrafast demagnetization of the structure is followed within the first picoseconds after excitation, and the recovery timescales on the order of 1 ps are in agreement with findings of ultrafast spectroscopy experiments in continuous films.

- We observe a non-adiabatic, homogeneous suppression of the magnetization field within the investigated time window; i.e., only the amplitude of the magnetization vector responds to the optically induced increase in spin temperature.

In addition, we find that, for short delay times, the Lorentz image contrast is a convolution of ultrafast demagnetization effects and electron scattering by transient near-fields. Therefore, one must perform a careful analysis of the contrast, if employing laser-pump and electron-probe pulses of similar duration.

The direct correspondence of image contrast and transient magnetization field, together with the versatility of adjusting temporal and spatial resolution in accordance with the sample system, makes fs-Lorentz microscopy a unique means for investigation of ultrafast magnetization dynamics in the nanoscale, when addressing questions both in the fundamental and technological levels.

\subsection{Discussion and Outlook}

\section{Imaging Ultrafast Magnetization Dynamics}

The work presented here demonstrates the potential of fs-Lorentz microscopy to image magnetization dynamics with hundreds-of-fs temporal resolution and below 100-nm spatial resolution. The need for imaging techniques able to address these time and length scales was highlighted a few years ago in a review article by pioneers in ultrafast magnetization dynamics [43]. 


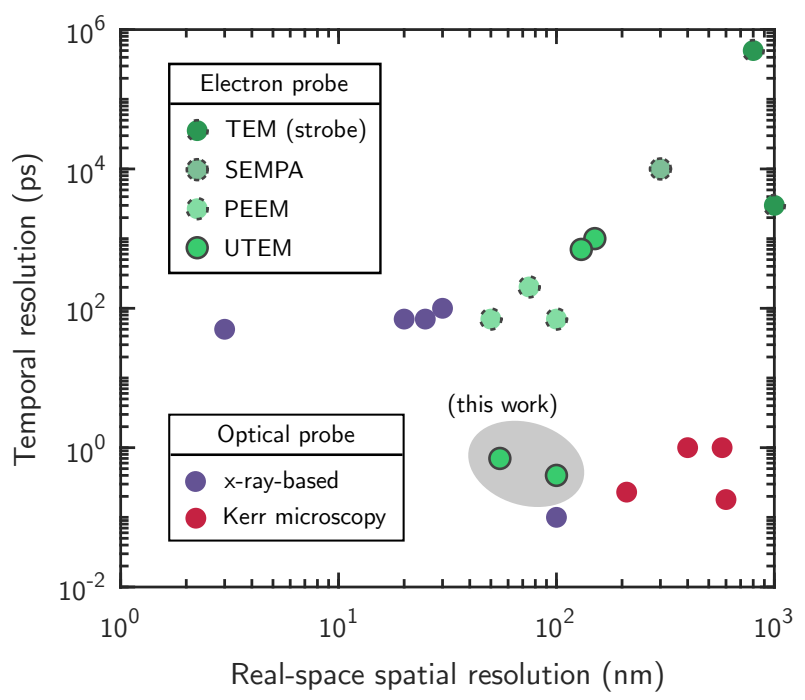

Fig. 6.1.: Comparison of spatial and temporal resolution achieved by different magnetic imaging techniques. All the methods make use of pulsed probe radiation, and the color code specifies the different kinds. For the references, see text.

Femtosecond-Lorentz microsocopy fulfill those requirements. In Figure 6.1, we compare the capabilities demonstrated in this work not only with alternative UTEM approaches $[16,18]$, but also with the first stroboscopic TEM [11, 12], scanning electron microscopy with polarization analysis (SEMPA) [17], photoemission electron microscopy [13-15], x-ray-based techniques in real space [2, 5, 8-10], and Kerr microscopy using visible/near-visible light $[1,3,4,7]$. We note that the spatiotemporal resolution achieved in this work is comparable only with Fourier transform holography using x-ray radiation of a free-electron laser source realized in large scale facilities. In comparison, our technique constitutes a lab-scale, widely accessible experimental tool.

A few further techniques are worth mentioning. Firstly, an all-electronic pump-probe scheme implemented in scanning tunneling microscopy [130] is able to measure spin relaxation times of single atoms on a ns timescale. Secondly, few-nm/tens-of-ps resolution is achieved in reciprocal space by using x-ray illumination at a free-electron laser facility [6]. In addition, a new approach for lab-scale photoemission electron microscopy demonstrated tens of nanometer resolution for magnetic imaging [131]. Lastly, nanoscale magnetic imaging was recently obtained by using a promising new concept for lab-scale extreme ultraviolet radiation [132]. They are not included in the comparison above because the former provides a very different kind of 
information, the second is not a real-space imaging technique and the last two have not yet demonstrated time resolution.

We now discuss possible investigations of ultrafast magnetization dynamics on the nanoscale enabled by our technique. Relevant processes occurring on femtosecondnanometer scales include the internal dynamics of domain walls (DWs), the exchange and spin-orbit interaction, and also the dipolar interactions in confined structures (see Figure 2.2). As an example, the optically induced broadening of DWs was observed in worm-like domain structures formed in multilayered magnetic films with strong perpendicular magnetization anisotropy, both in reciprocal-space [6] and in real-space [8]. The broadening was attributed to superdiffusive spin transport of the excited spins on a 300-fs timescale [60]. Real-space imaging of such a process in nanoscale geometries can now be performed using fs-Lorentz microscopy.

The magnetic vortex investigated in our work is one of many interesting topological spin textures; others are skyrmions [20, 133-135], merons [136, 137] and Bloch points $[35,138,139]$. The great interest in technological applications of these textures (for instance, racetrack memories [140, 141] and energy storage [142]) has prompted investigations on the dynamical response of skyrmions to several stimuli [9, 101-103, 143-146], and of Bloch points to magnetic field pulses [147], but not yet with a combined sub-ps temporal and tens-of-nm spatial resolution. Given the small dimensions of such structures, exploring them with the high spatial resolution of fs-Lorentz microscopy may bring new perspectives.

The original design for racetrack memory devices involved moving domain walls (DWs) along thin stripes [148], and concepts for achieving higher tracking speeds include the use of chiral DWs $[149,150]$ or modulated material sections in nanowires for 3D implementations [151]. Especially for the last concept, a high spatial resolution imaging technique such as fs-Lorentz microscopy is essential to visualize the dynamical response of DWs.

Our technique contributes a new tool for investigating further phenomena related to the interaction of spins with intense light fields. We note that, due to the nm-spatial resolution achieved in real-space images using fs-Lorentz microscopy, heterogeneous sample systems can be investigated, such as isolated magnetic nanoislands (Section 5.1) and nanostructured magnetic films. As an example, an optical standing 
wave formed inside an elliptical mask spatially confines ultrafast demagnetization [8]. The inhomogeneous excitation produced by near-field localization in such nanostructures may trigger intriguing magnetic phenomena still to be explored.

A further possible research direction using our technique concerns the dependence of ultrafast magnetization dynamics processes on the dimensions and shape of the magnetic elements. On longer timescales of picoseconds, for example, the magnetic damping parameter of nanostructures was found to be influenced by their size (isolated islands) [152] and shape (array) [153]. Studies for determining whether similar control occurs on shorter timescales can also benefit from the combined spatiotemporal resolution of fs-Lorentz microscopy.

\section{Improving Detection Capabilities}

As we have shown in Chapter 3, one of the main challenges in fs-Lorentz microscopy is the trade-off between signal within a coherently illuminated area and shortest exposure times to avoid a drift-reduced spatial resolution. Therefore, better detection capabilities can significantly improve the potential of fs-Lorentz microscopy, and other phase-contrast techniques in UTEM.

Recently, a new detector has been installed in the Göttingen UTEM instrument: a direct-electron detector device (DDD, model DE-16), part of a new class of sensors for TEM. The main difference from the predecessor generation of CCD sensors is a direct sensitivity to the electrons, with no need of a scintillator. As a result, lower doses are required to form an image, and spatial resolution is improved ${ }^{1}[30,154$, $155]$.

Figure 6.2 demonstrates a comparison between the two detectors, showing a pair of Lorentz micrographs obtained using electron pulses at the same illumination conditions, fixed microscope parameters and same total exposure time. As usually, short-exposure images were acquired, and the frame-to-frame drift was corrected; a gaussian filter was applied to the final image (cf. Section 3.3.2). For better visualization of the different performances, we use here electron pulses at a lower

\footnotetext{
${ }^{1}$ For CCD-based detectors, the conversion to photons takes place in a scintillator, and the photons are transferred to the CCD surface through an array of optical fibers. An unavoidable delocalization of the signal impinging on one scintillator "pixel" results in a broadened pixel at the CCD, and a degraded spatial resolution.
} 


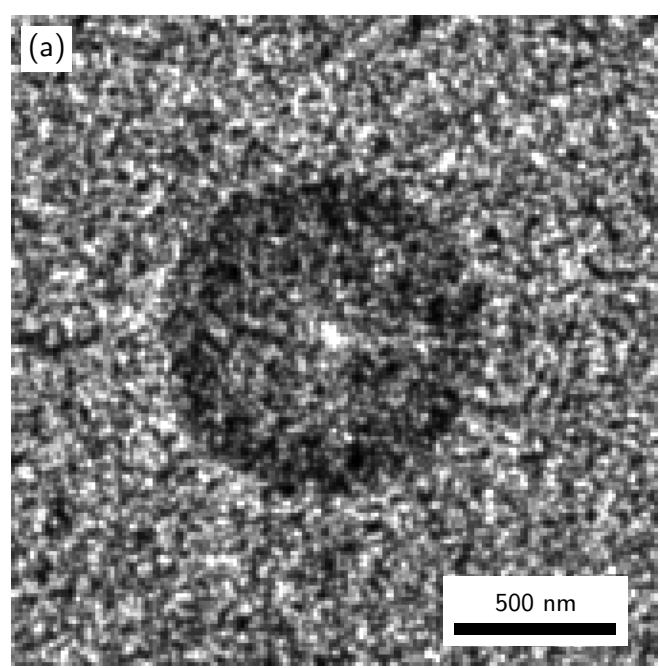

UltraScan (CCD)

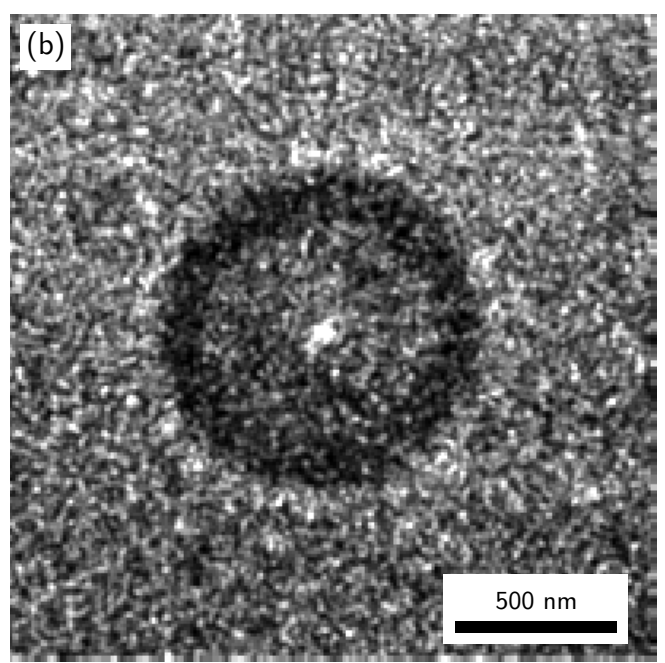

DE16 (DDD)

Fig. 6.2.: Comparison between different detectors. (a,b) Lorentz micrographs at a defocus of $-3 \mathrm{~mm}$ obtained using 700 -fs electron pulses at 250-kHz repetition rate, recorded on a (a) CCD-based sensor, and on a (b) DDD sensor. Images consist of $5 \times 60$ sexposures in (a), and of $30 \times 10$ s-exposures in (b).

repetition rate than in most of the thesis. In addition, we note that, due to the varied detector sensitivities, the individual frames were accumulated for $10 \mathrm{~s}$ for the DDD, and $60 \mathrm{~s}$ for the CCD. The improvement in contrast and spatial resolution is remarkable.

In summary, with enhanced detection capabilities, the spatial resolution of fs-Lorentz microscopy can be further improved.

\section{Other Phase-contrast Applications}

Lorentz microscopy is also sensitive to spatial variations of the electric potential (see Equation 2.1 and Section 2.2). Therefore, it is in principle possible to visualize transient electric fields in defocused micrographs.

Other ultrafast phase-contrast imaging techniques can build upon fs-Lorentz microscopy. For instance, the better characterization of the illumination coherence length / spatial resolution trade-offs, together with the enhanced detection possibilities presented above, will enable the development of ultrafast holography. We note that the main historical obstacle for the implementation of non-time resolved electron holography was low-brightness electron sources. Having demonstrated 
holograms in the UTEM with cw-laser triggered photoemission [28], we anticipate the establishment of holography with high temporal resolution.

The sensitivity to electric and magnetic fields of both Lorentz microscopy and holography, now with combined nanometer and hundreds of femtoseconds resolution, will facilitate unprecedented opportunities to investigate fields at, e.g., interfaces and at the vicinity of nanostructures.

To conclude, the work reported here is a first realization of high-sensitivity phasecontrast imaging in Ultrafast Transmission Electron Microscopy. The advent of high-brightness, ultrafast pulsed electron emitters will enable the visualization of a multitude of intriguing dynamical phenomena on the nanoscale. 



\section{Samples Crystalline Structure}

We characterize the crystalline structure of the prepared samples using diffraction and dark-field imaging at a conventional transmission electron microscope (JEOL2010F, field emission gun at $200 \mathrm{kV}$ for the lithography samples; FEI/Philips-CM30, $\mathrm{LaB}_{6}$ thermionic gun at $300 \mathrm{kV}$ for the FIB samples). From the diffraction pattern, the crystalline structure of a material is determined, while a dark-field image directly yields the crystal grain size [62]. Moreover, by using a 100-nm-sized electron beam, these methods can be easily used to characterize a single nanoisland. In Figure A.1, a set of bright-field image, dark-field image and diffraction pattern is shown for discs prepared by electron-beam lithography/lift-off and by FIB patterning.

The ring-like diffraction patterns in Figures A.1c,f reveal that the prepared samples are polycrystalline. The crystallites present a face-centered cubic crystal structure, as usually found in permalloy $[156,157]$. The more speckled pattern of the FIBpatterned disc indicates a larger grain size than the ones of the lithographically prepared structures. This can be quantitatively evaluated by looking at the dark-field images in Figures A.1b,e, from which we extract an average grain size of 20-30 nm for the FIB preparation method, and smaller than $10 \mathrm{~nm}$ for the lithography preparation method.

The modification of the crystalline structure of permalloy by Galium implantation in FIB, and the consequences for its magnetic properties, were investigated by Ozkayal and coworkers [157]. Although not using FIB to pattern nanostructures, they found that for high ion beam doses the crystal grains get larger, which may lead to stress at the boundaries of the exposed area. The stress in turn induces pinning of domain walls, increasing the coercivity. In the disc fabricated here, the increased coercivity and domain wall pinning may affect the formation of the magnetic vortex ${ }^{1}$ and the reversibility after partial demagnetization by the laser pulse.

\footnotetext{
${ }^{1}$ The stray-field contribution to the total free energy remains the same. The crystalline anisotropy contribution, however, induces the spin alignment within each larger crystallite independently of the others (see Section 2.1.1). Consequently, the spin alignment to the disc edges does not necessarily minimize the total free energy.
} 

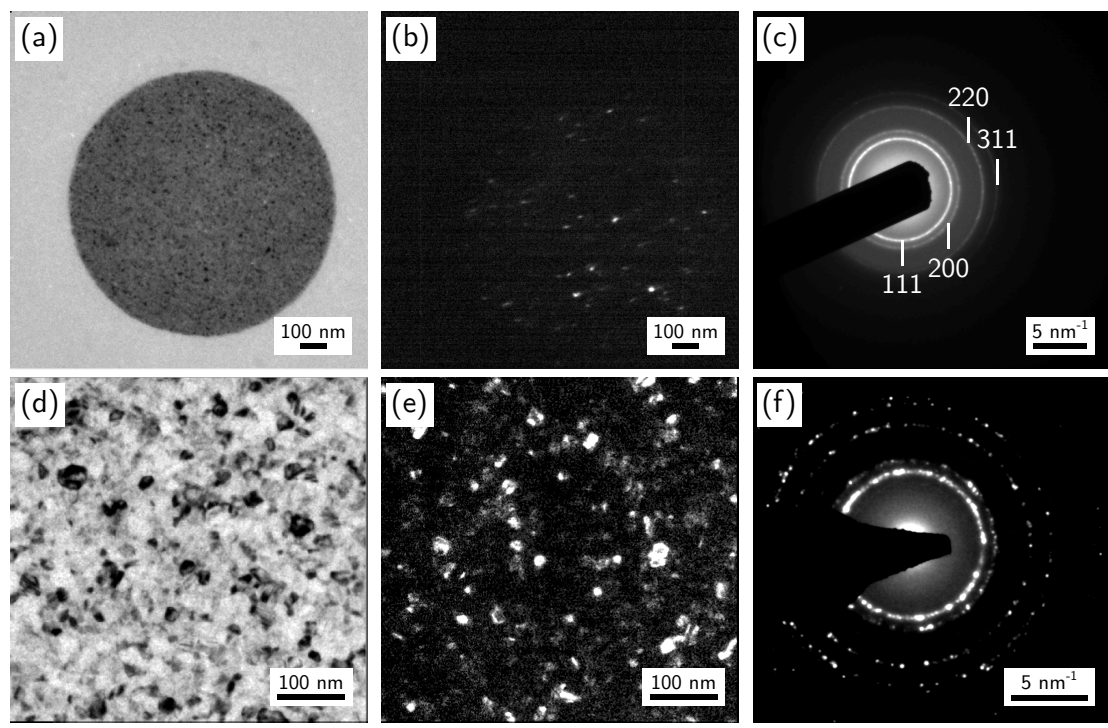

Fig. A.1.: Characterization of the crystalline structure of as-prepared samples. (a) Brightfield image, (b) dark-field image, and (c) diffraction pattern of a disc prepared by electron-beam lithography/lift-off. (d) Bright-field image, (e) dark-field image, and $(f)$ diffraction pattern of a disc prepared by FIB patterning.

We observed crystal grain growth also as a result of prolonged exposure to 800-nm pulsed laser illumination (as mentioned in Chapter 3), as can be seen in the diffraction patterns shown in Figures A.2c,d. From the dark areas within the discs in the bright-field images (Figures A.2a,b), we can estimate an increase in grain size of up to $30-40 \mathrm{~nm}$ after exposure to laser illumination of $11.9-\mathrm{mJ} / \mathrm{cm}^{2}$ fluence at $250 \mathrm{kHz}$, and up to $90-100 \mathrm{~nm}$ after exposure to laser of $19.8-\mathrm{mJ} / \mathrm{cm}^{2}$ fluence at $500 \mathrm{kHz}$. 

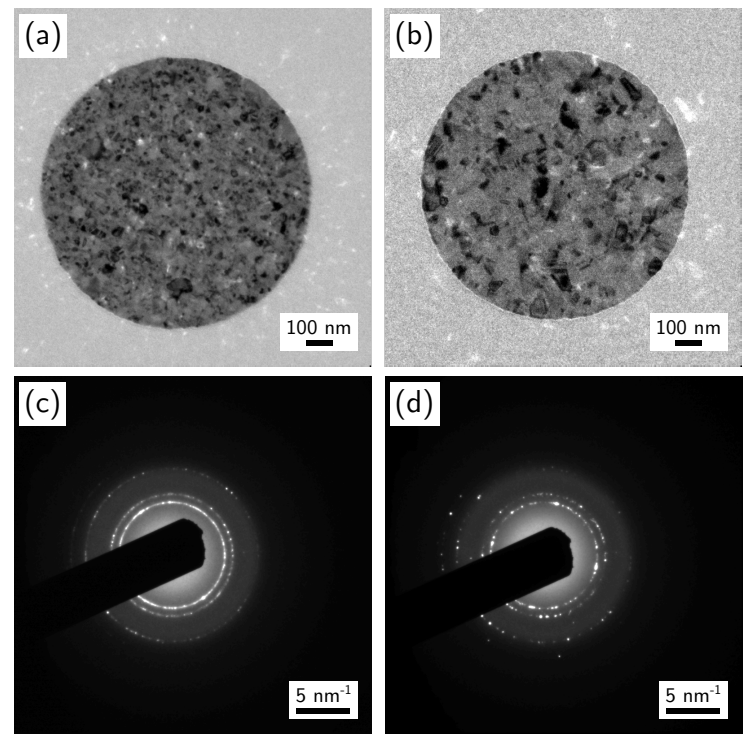

Fig. A.2.: Crystal grain growth after prolonged exposure to high-fluence laser. (a) Brightfield image, and (c) diffraction pattern of a disc exposed to a pump fluence of $11.9 \mathrm{~mJ} / \mathrm{cm}^{2}$ at $250 \mathrm{kHz}$. (b) Bright-field image, and (d) diffraction pattern of a disc exposed to a pump fluence of $19.8 \mathrm{~mJ} / \mathrm{cm}^{2}$ at $500 \mathrm{kHz}$. 



\section{Simulations Details}

The simulation grid parameters were chosen taking into account the experimental spatial resolution and the computation time, especially for the incoherent sum approach, for which 2000 intermediate images are computed for composing a single, final image. At these conditions, the time for simulating a single image using a

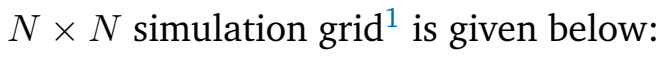

\begin{tabular}{||c|c||}
\hline$N$ & computation time (s) \\
\hline \hline $2^{9}$ & 48.6 \\
$2^{10}$ & 223.0 \\
$2^{11}$ & 903.9 \\
\hline
\end{tabular}

We note that the actual exit wave function $\psi(\vec{r})$ occupies only the central $N / 2 \times N / 2$ area of the grid. The remaining area is occupied by replicating the edge values of $\psi(\vec{r})$. This procedure reduces non-physical edge effects on the area of interest, and improve the quality of the simulation for lower spatial frequencies (see Equation B.2).

Since the simulations usually involved a parameter series (for instance, defocus or magnetic induction), a simulation grid of $N=2^{9}$ was chosen for reducing simulation time. For a pixel size of $9.3 \mathrm{~nm}$ (experimental pixel size at the minimum used defocus of $-1.5 \mathrm{~mm}$ ), the grid length is then $2380 \mathrm{~nm}$, which is adequate to contain the magnetic disc of $1-\mu \mathrm{m}$ diameter.

In accordance to Equation 4.6, we make use of the Discrete Fourier Transform for the numerical simulations. To avoid aliasing, we must then fulfill the Nyquist requirement [158], both for the forward Fourier transform of the wave function $\psi(\vec{r})$, and for the inverse transform of the product $T(\vec{q}) \mathcal{F}[\psi(\vec{r})]$. To this end, the sampling rate must exceed (or be equal to) the Nyquist rate, defined as $\pi / P$, where $P$ is the pixel size.

\footnotetext{
${ }^{1}$ A simulation grid with $N=2^{n}$ is ideal for Fast Fourier Transform (FFT) algorithms (such as the one applied here).
} 
In the forward direction, we can hence define a maximum spatial frequency $q_{x, \max }$ from the real-space pixel $\Delta x$ :

$$
q_{x, \max }=\frac{1}{2 \pi} \frac{\pi}{\Delta x}
$$

Since the spatial frequency grid has the same number of pixels as the spatial grid, we find the spatial frequency "pixel" $\Delta q_{x}$ (similarly for $\Delta q_{y}$ ) to be:

$$
\Delta q_{x}=\frac{2 q_{x, \max }}{N}=\frac{1}{N \Delta x}
$$

For the inverse transform, however, we need also to ensure the contrast transfer function $T(\vec{q})=\mathrm{e}^{-\mathrm{i} \chi(\vec{q})}$ is properly sampled in Fourier space. By recognizing that $T(\vec{q})$ presents an oscillatory behaviour with ever increasing spatial frequencies (see Figure 4.4), the Nyquist requirement, here, relates to the maximum rate at which $\chi(\vec{q})$ changes. Therefore, we find, for the $q_{x}$-direction (similarly for $q_{y}$-direction) $[159,160]:$

$$
\left|\frac{\mathrm{d} \chi}{\mathrm{d} q_{x}}\right|_{\max } \leq \frac{\pi}{\Delta q_{x}} .
$$

We now consider only the most important contribution for Lorentz images (i.e. the defocus term). By noticing that the derivative of $\chi(\vec{q})$ is maximum for $q_{x, \max }$, and by using $\Delta q_{x}=\frac{1}{N \Delta x}$, we get:

$$
\begin{gathered}
\frac{\pi|\Delta f| \lambda}{\Delta x} \leq \pi N \Delta x, \\
|\Delta f| \leq \frac{N(\Delta x)^{2}}{\lambda},
\end{gathered}
$$

which means that, for a given discretization grid defined by $N, \Delta x$ and $\Delta y$, there is a maximum defocus $|\Delta f|$ for which the numerical simulation is still valid.

For all the simulations in this thesis, we adopt a $N \times N$ grid with $N=2^{9}$, and squared pixels of $\Delta x=\Delta y=10 \mathrm{~nm}$. Such grid allows us to simulate images using defocus of up to $\pm 15 \mathrm{~mm}$. 


\title{
Symbols and Abbreviations
}

\author{
Abbreviations \\ 3TM three-temperature model \\ BFP back-focal plane \\ CCD charge-coupled device \\ cW continuous wave \\ DDD direct-electron device detector \\ DTEM dynamic transmission electron microscopy \\ DW domain wall \\ FIB focused-ion beam \\ FWHM full-width at half-maximum \\ PEEM photoemission electron microscopy \\ SEMPA scanning electron microscopy with polarization analysis \\ SiN silicon nitride \\ SNR signal-to-noise ratio \\ TEM transmission electron microscopy \\ UTEM ultrafast transmission electron microscopy \\ Greek Symbols \\ $\beta \quad$ ratio of $v$ to the speed of light \\ $\gamma \quad$ Lorentz factor \\ $\lambda \quad$ electron wavelength \\ $\mu_{s c} \quad$ degree of spatial coherence \\ $\mu_{t c} \quad$ degree of temporal coherence \\ $\sigma_{\theta} \quad$ illumination angular spread
}




$\begin{array}{ll}\phi_{0} & \text { magnetic flux quantum } \\ \phi(\vec{r}) & \text { phase of electron wave function } \\ \chi(\vec{q}) & \text { coherent aberration function } \\ \psi(\vec{r}) & \text { electron wave function }\end{array}$

\section{Symbols}

$\vec{A} \quad$ magnetic vector potential

$B_{0} \quad$ magnetic induction

$C_{c} \quad$ chromatic aberration coefficient

$C_{s} \quad$ spherical aberration coefficient

$E^{s c} \quad$ spatial coherence envelope function

$E^{t c} \quad$ temporal coherence envelope function

$\Delta f \quad$ defocus

$f_{\text {rep }} \quad$ repetition rate

$\mathcal{F} \quad$ Fourier transform

$k \quad$ electron wavenumber

$l_{\perp} \quad$ transverse coherence length

$l_{\|} \quad$ longitudinal coherence length

$\vec{m} \quad$ reduced magnetization vector

$\vec{M} \quad$ magnetization field

$M_{\mathrm{S}} \quad$ saturation magnetization

$N_{e l} \quad$ number of electrons

$\vec{q} \quad$ reciprocal space vector (two-dimensional)

$\vec{r} \quad$ real space vector (two-dimensional)

$t, \Delta t \quad$ time, delay time

$T(\vec{q}) \quad$ contrast transfer function

$T_{\mathrm{Cu}} \quad$ Curie temperature

$v \quad$ electron speed

$V \quad$ electrostatic potential 


\section{Bibliography}

[1] Y. Acremann, C. H. Back, M. Buess, O. Portmann, A. Vaterlaus, D. Pescia, and H. Melchior. "Imaging Precessional Motion of the Magnetization Vector". Science 290 (2000), pp. 492-495 (cit. on pp. 1, 91).

[2] B. Van Waeyenberge, A. Puzic, H. Stoll, K. W. Chou, T. Tyliszczak, R. Hertel, M. Fähnle, H. Brückl, K. Rott, G. Reiss, I. Neudecker, D. Weiss, C. H. Back, and G. Schütz. "Magnetic Vortex Core Reversal by Excitation with Short Bursts of an Alternating Field". Nature 444 (2006), pp. 461-464 (cit. on pp. 1, 91).

[3] A. Laraoui, M. Albrecht, and J.-Y. Bigot. "Femtosecond Magneto-Optical Kerr Microscopy". Opt. Lett., OL 32 (2007), pp. 936-938 (cit. on pp. 1, 91).

[4] J. Li, M.-S. Lee, W. He, B. Redeker, A. Remhof, E. Amaladass, C. Hassel, and T. Eimüller. "Magnetic Imaging with Femtosecond Temporal Resolution". Review of Scientific Instruments 80 (2009), p. 073703 (cit. on pp. 1, 91).

[5] H. Jung, Y.-S. Yu, K.-S. Lee, M.-Y. Im, P. Fischer, L. Bocklage, A. Vogel, M. Bolte, G. Meier, and S.-K. Kim. "Observation of Coupled Vortex Gyrations by 70-Ps-Time- and 20-Nm-Space-Resolved Full-Field Magnetic Transmission Soft x-Ray Microscopy". Appl. Phys. Lett. 97 (2010), p. 222502 (cit. on pp. 1, 91).

[6] B. Pfau, S. Schaffert, L. Müller, C. Gutt, A. Al-Shemmary, F. Büttner, R. Delaunay, S. Düsterer, S. Flewett, R. Frömter, J. Geilhufe, E. Guehrs, C. M. Günther, R. Hawaldar, M. Hille, N. Jaouen, A. Kobs, K. Li, J. Mohanty, H. Redlin, W. F. Schlotter, D. Stickler, R. Treusch, B. Vodungbo, M. Kläui, H. P. Oepen, J. Lüning, G. Grübel, and S. Eisebitt. "Ultrafast Optical Demagnetization Manipulates Nanoscale Spin Structure in Domain Walls". Nat. Commun. 3 (2012), p. 1100 (cit. on pp. 1, 91, 92).

[7] B. Mozooni, T. von Hofe, and J. McCord. "Picosecond Wide-Field Magneto-Optical Imaging of Magnetization Dynamics of Amorphous Film Elements". Phys. Rev. B 90 (2014), p. 054410 (cit. on pp. 1, 91).

[8] C. von Korff Schmising, B. Pfau, M. Schneider, C. M. Günther, M. Giovannella, J. Perron, B. Vodungbo, L. Müller, F. Capotondi, E. Pedersoli, N. Mahne, J. Lüning, and S. Eisebitt. "Imaging Ultrafast Demagnetization Dynamics after a Spatially Localized Optical Excitation". Phys. Rev. Lett. 112 (2014) (cit. on pp. 1, 91-93).

[9] F. Büttner, C. Moutafis, M. Schneider, B. Krüger, C. M. Günther, J. Geilhufe, C. v. K. Schmising, J. Mohanty, B. Pfau, S. Schaffert, A. Bisig, M. Foerster, T. Schulz, C. a. F. Vaz, J. H. Franken, H. J. M. Swagten, M. Kläui, and S. Eisebitt. "Dynamics and Inertia of Skyrmionic Spin Structures". Nat. Phys. 11 (2015), pp. 225-228 (cit. on pp. 1, 91, 92). 
[10] A. Bisig, C. A. Akosa, J.-H. Moon, J. Rhensius, C. Moutafis, A. von Bieren, J. Heidler, G. Kiliani, M. Kammerer, M. Curcic, M. Weigand, T. Tyliszczak, B. Van Waeyenberge, H. Stoll, G. Schütz, K.-J. Lee, A. Manchon, and M. Kläui. "Enhanced Nonadiabaticity in Vortex Cores Due to the Emergent Hall Effect". Phys. Rev. Lett. 117 (2016), p. 277203 (cit. on pp. 1, 91).

[11] O. Bostanjoglo and T. Rosin. "Resonance Oscillations of Magnetic Domain Walls and Bloch Lines Observed by Stroboscopic Electron Microscopy". Phys. Status Solidi (a) 57 (1980), pp. 561-568 (cit. on pp. 1, 28-30, 91).

[12] V. I. Petrov and G. V. Spivak. "On a Stroboscopic Lorentz Microscopy". Z. angew. Physik 27 (1969), pp. 188-191 (cit. on pp. 1, 28, 29, 91).

[13] S.-B. Choe, Y. Acremann, A. Scholl, A. Bauer, A. Doran, J. Stöhr, and H. A. Padmore. "Vortex Core-Driven Magnetization Dynamics". Science 304 (2004), pp. 420-422 (cit. on pp. 1, 91).

[14] L. Le Guyader, A. Kleibert, A. Fraile Rodríguez, S. El Moussaoui, A. Balan, M. Buzzi, J. Raabe, and F. Nolting. "Studying Nanomagnets and Magnetic Heterostructures with X-Ray PEEM at the Swiss Light Source". Journal of Electron Spectroscopy and Related Phenomena 185 (2012), pp. 371-380 (cit. on pp. 1, 91).

[15] S. Finizio, S. Wintz, D. Bracher, E. Kirk, A. S. Semisalova, J. Förster, K. Zeissler, T. Weßels, M. Weigand, K. Lenz, A. Kleibert, and J. Raabe. "Thick Permalloy Films for the Imaging of Spin Texture Dynamics in Perpendicularly Magnetized Systems". Phys. Rev. B 98 (2018), p. 104415 (cit. on pp. 1, 91).

[16] H. S. Park, J. S. Baskin, and A. H. Zewail. "4D Lorentz Electron Microscopy Imaging: Magnetic Domain Wall Nucleation, Reversal, and Wave Velocity". Nano Lett. 10 (2010), pp. 3796-3803 (cit. on pp. 1, 2, 29-31, 91).

[17] R. Frömter, F. Kloodt, S. Rößler, A. Frauen, P. Staeck, D. R. Cavicchia, L. Bocklage, V. Röbisch, E. Quandt, and H. P. Oepen. "Time-Resolved Scanning Electron Microscopy with Polarization Analysis". Appl. Phys. Lett. 108 (2016), p. 142401 (cit. on pp. 1, 91).

[18] K. B. Schliep, P. Quarterman, J.-P. Wang, and D. J. Flannigan. "Picosecond Fresnel Transmission Electron Microscopy". Appl. Phys. Lett. 110 (2017), p. 222404 (cit. on pp. 1, 2, 29-31, 91).

[19] J. N. Chapman. "The Investigation of Magnetic Domain Structures in Thin Foils by Electron Microscopy". J. Phys. D: Appl. Phys. 17 (1984), p. 623 (cit. on p. 1).

[20] X. Z. Yu, Y. Onose, N. Kanazawa, J. H. Park, J. H. Han, Y. Matsui, N. Nagaosa, and Y. Tokura. "Real-Space Observation of a Two-Dimensional Skyrmion Crystal". Nature 465 (2010), pp. 901-904 (cit. on pp. 1, 92).

[21] S. D. Pollard, L. Huang, K. S. Buchanan, D. A. Arena, and Y. Zhu. "Direct Dynamic Imaging of Non-Adiabatic Spin Torque Effects". Nat. Commun. 3 (2012), p. 1028 (cit. on p. 1).

[22] K. Koike. "Spin-Polarized Scanning Electron Microscopy". Microscopy 62 (2013), pp. 177-191 (cit. on p. 1).

[23] A. H. Zewail. "Four-Dimensional Electron Microscopy". Science 328 (2010), pp. 187193 (cit. on pp. 1, 28). 
[24] L. Piazza, D. J. Masiel, T. LaGrange, B. W. Reed, B. Barwick, and F. Carbone. "Design and Implementation of a Fs-Resolved Transmission Electron Microscope Based on Thermionic Gun Technology". Chemical Physics 423 (2013), pp. 79-84 (cit. on pp. 1, 28).

[25] E. Kieft, K. B. Schliep, P. K. Suri, and D. J. Flannigan. "Communication: Effects of Thermionic-Gun Parameters on Operating Modes in Ultrafast Electron Microscopy". Structural Dynamics 2 (2015), p. 051101 (cit. on pp. 1, 28).

[26] G. Cao, S. Sun, Z. Li, H. Tian, H. Yang, and J. Li. "Clocking the Anisotropic Lattice Dynamics of Multi-Walled Carbon Nanotubes by Four-Dimensional Ultrafast Transmission Electron Microscopy". Sci Rep 5 (2015), p. 8404 (cit. on pp. 1, 28).

[27] A. Feist, K. E. Echternkamp, J. Schauss, S. V. Yalunin, S. Schäfer, and C. Ropers. "Quantum Coherent Optical Phase Modulation in an Ultrafast Transmission Electron Microscope". Nature 521 (2015), pp. 200-203 (cit. on pp. 1, 32, 49).

[28] A. Feist, N. Bach, N. Rubiano da Silva, T. Danz, M. Möller, K. E. Priebe, T. Domröse, J. G. Gatzmann, S. Rost, J. Schauss, S. Strauch, R. Bormann, M. Sivis, S. Schäfer, and C. Ropers. "Ultrafast Transmission Electron Microscopy Using a Laser-Driven Field Emitter: Femtosecond Resolution with a High Coherence Electron Beam". Ultramicroscopy 176 (2017), pp. 63-73 (cit. on pp. 1, 2, 28-30, 32, 35-37, 45, 49, $51,83,95)$.

[29] J. S. Kim, T. LaGrange, B. W. Reed, M. L. Taheri, M. R. Armstrong, W. E. King, N. D. Browning, and G. H. Campbell. "Imaging of Transient Structures Using Nanosecond in Situ TEM". Science 321 (2008), pp. 1472-1475 (cit. on pp. 1, 28).

[30] Y. M. Lee, Y. J. Kim, Y.-J. Kim, and O.-H. Kwon. "Ultrafast Electron Microscopy Integrated with a Direct Electron Detection Camera". Structural Dynamics 4 (2017), p. 044023 (cit. on pp. 1, 28, 93).

[31] P. Hommelhoff, Y. Sortais, A. Aghajani-Talesh, and M. A. Kasevich. "Field Emission Tip as a Nanometer Source of Free Electron Femtosecond Pulses". Phys. Rev. Lett. 96 (2006), p. 077401 (cit. on p. 2).

[32] C. Ropers, D. R. Solli, C. P. Schulz, C. Lienau, and T. Elsaesser. "Localized Multiphoton Emission of Femtosecond Electron Pulses from Metal Nanotips". Phys. Rev. Lett. 98 (2007), p. 043907 (cit. on p. 2).

[33] B. Barwick, C. Corder, J. Strohaber, N. Chandler-Smith, C. Uiterwaal, and H. Batelaan. "Laser-Induced Ultrafast Electron Emission from a Field Emission Tip". New J. Phys. 9 (2007), pp. 142-142 (cit. on p. 2).

[34] E. Beaurepaire, J.-C. Merle, A. Daunois, and J.-Y. Bigot. "Ultrafast Spin Dynamics in Ferromagnetic Nickel". Phys. Rev. Lett. 76 (1996), pp. 4250-4253 (cit. on pp. 5, 9, 10).

[35] A. Hubert and R. Schäfer. Magnetic Domains - The Analysis of Magnetic Microstructures. Heidelberg: Springer, 2000 (cit. on pp. 5-7, 59, 92).

[36] A. Kirilyuk, A. V. Kimel, and T. Rasing. "Ultrafast Optical Manipulation of Magnetic Order”. Rev. Mod. Phys. 82 (2010), pp. 2731-2784 (cit. on pp. 5, 7, 10).

[37] A. Vansteenkiste, J. Leliaert, M. Dvornik, M. Helsen, F. Garcia-Sanchez, and B. Van Waeyenberge. "The Design and Verification of MuMax3". AIP Adv. 4 (2014), p. 107133 (cit. on pp. 6, 81). 
[38] N. A. Usov and S. E. Peschany. "Magnetization Curling in a Fine Cylindrical Particle". J. Magn. Magn. Mater. 118 (1993), pp. L290-L294 (cit. on pp. 7, 38, 71).

[39] T. Shinjo, T. Okuno, R. Hassdorf, K. Shigeto, and T. Ono. "Magnetic Vortex Core Observation in Circular Dots of Permalloy". Science 289 (2000), pp. 930-932 (cit. on pp. 7, 38, 59).

[40] A. Wachowiak, J. Wiebe, M. Bode, O. Pietzsch, M. Morgenstern, and R. Wiesendanger. "Direct Observation of Internal Spin Structure of Magnetic Vortex Cores". Science 298 (2002), pp. 577-580 (cit. on p. 7).

[41] A. Hubert and M. Rührig. "Micromagnetic Analysis of Thin-film Elements (Invited)". Journal of Applied Physics 69 (1991), pp. 6072-6077 (cit. on p. 7).

[42] R. Hertel, S. Gliga, M. Fähnle, and C. M. Schneider. "Ultrafast Nanomagnetic Toggle Switching of Vortex Cores". Phys. Rev. Lett. 98 (2007), p. 117201 (cit. on p. 7).

[43] J.-Y. Bigot and M. Vomir. "Ultrafast Magnetization Dynamics of Nanostructures". Annalen der Physik 525 (2013), pp. 2-30 (cit. on pp. 7, 8, 10, 90).

[44] C. D. Stanciu, F. Hansteen, A. V. Kimel, A. Kirilyuk, A. Tsukamoto, A. Itoh, and T. Rasing. "All-Optical Magnetic Recording with Circularly Polarized Light". Phys. Rev. Lett. 99 (2007), p. 047601 (cit. on pp. 8, 9).

[45] K. Vahaplar, A. M. Kalashnikova, A. V. Kimel, D. Hinzke, U. Nowak, R. Chantrell, A. Tsukamoto, A. Itoh, A. Kirilyuk, and T. Rasing. "Ultrafast Path for Optical Magnetization Reversal via a Strongly Nonequilibrium State". Phys. Rev. Lett. 103 (2009), p. 117201 (cit. on p. 8).

[46] D. Steil, S. Alebrand, A. Hassdenteufel, M. Cinchetti, and M. Aeschlimann. "AllOptical Magnetization Recording by Tailoring Optical Excitation Parameters". Phys. Rev. B 84 (2011), p. 224408 (cit. on p. 8).

[47] T. A. Ostler, J. Barker, R. F. L. Evans, R. W. Chantrell, U. Atxitia, O. ChubykaloFesenko, S. El Moussaoui, L. Le Guyader, E. Mengotti, L. J. Heyderman, F. Nolting, A. Tsukamoto, A. Itoh, D. Afanasiev, B. A. Ivanov, A. M. Kalashnikova, K. Vahaplar, J. Mentink, A. Kirilyuk, T. Rasing, and A. V. Kimel. "Ultrafast Heating as a Sufficient Stimulus for Magnetization Reversal in a Ferrimagnet". Nat. Commun. 3 (2012), p. 666 (cit. on p. 8).

[48] S. Mangin, M. Gottwald, C.-H. Lambert, D. Steil, V. Uhlî́, L. Pang, M. Hehn, S. Alebrand, M. Cinchetti, G. Malinowski, Y. Fainman, M. Aeschlimann, and E. E. Fullerton. "Engineered Materials for All-Optical Helicity-Dependent Magnetic Switching". Nat. Mater. 13 (2014), pp. 286-292 (cit. on p. 8).

[49] C.-H. Lambert, S. Mangin, B. S. D. C. S. Varaprasad, Y. K. Takahashi, M. Hehn, M. Cinchetti, G. Malinowski, K. Hono, Y. Fainman, M. Aeschlimann, and E. E. Fullerton. "All-Optical Control of Ferromagnetic Thin Films and Nanostructures". Science 345 (2014), pp. 1337-1340 (cit. on p. 8).

[50] T.-M. Liu, T. Wang, A. H. Reid, M. Savoini, X. Wu, B. Koene, P. Granitzka, C. E. Graves, D. J. Higley, Z. Chen, G. Razinskas, M. Hantschmann, A. Scherz, J. Stöhr, A. Tsukamoto, B. Hecht, A. V. Kimel, A. Kirilyuk, T. Rasing, and H. A. Dürr. "Nanoscale Confinement of All-Optical Magnetic Switching in TbFeCo - Competition with Nanoscale Heterogeneity". Nano Lett. 15 (2015), pp. 6862-6868 (cit. on p. 8). 
[51] A. Stupakiewicz, K. Szerenos, D. Afanasiev, A. Kirilyuk, and A. V. Kimel. "Ultrafast Nonthermal Photo-Magnetic Recording in a Transparent Medium". Nature 542 (2017), pp. 71-74 (cit. on p. 8).

[52] R. John, M. Berritta, D. Hinzke, C. Müller, T. Santos, H. Ulrichs, P. Nieves, J. Walowski, R. Mondal, O. Chubykalo-Fesenko, J. McCord, P. M. Oppeneer, U. Nowak, and M. Münzenberg. "Magnetisation Switching of FePt Nanoparticle Recording Medium by Femtosecond Laser Pulses". Sci. Rep. 7 (2017), p. 4114 (cit. on p. 8).

[53] T. Eggebrecht, M. Möller, J. G. Gatzmann, N. Rubiano da Silva, A. Feist, U. Martens, H. Ulrichs, M. Münzenberg, C. Ropers, and S. Schäfer. "Light-Induced Metastable Magnetic Texture Uncovered by in Situ Lorentz Microscopy". Phys. Rev. Lett. 118 (2017), p. 097203 (cit. on pp. 8, 9, 36, 57).

[54] X. Fu, S. D. Pollard, B. Chen, B.-K. Yoo, H. Yang, and Y. Zhu. "Optical Manipulation of Magnetic Vortices Visualized in Situ by Lorentz Electron Microscopy". Sci. Adv. 4 (2018), eaat3077 (cit. on p. 8).

[55] G. L. Eesley. "Observation of Nonequilibrium Electron Heating in Copper". Phys. Rev. Lett. 51 (1983), pp. 2140-2143 (cit. on p. 9).

[56] H. E. Elsayed-Ali, T. B. Norris, M. A. Pessot, and G. A. Mourou. "Time-Resolved Observation of Electron-Phonon Relaxation in Copper". Phys. Rev. Lett. 58 (1987), pp. 1212-1215 (cit. on p. 9).

[57] R. W. Schoenlein, W. Z. Lin, J. G. Fujimoto, and G. L. Eesley. "Femtosecond Studies of Nonequilibrium Electronic Processes in Metals". Phys. Rev. Lett. 58 (1987), pp. 1680-1683 (cit. on p. 9).

[58] F. Hansteen, A. Kimel, A. Kirilyuk, and T. Rasing. "Femtosecond Photomagnetic Switching of Spins in Ferrimagnetic Garnet Films". Phys. Rev. Lett. 95 (2005), p. 047402 (cit. on p. 10).

[59] J.-Y. Bigot, M. Vomir, and E. Beaurepaire. "Coherent Ultrafast Magnetism Induced by Femtosecond Laser Pulses". Nat. Phys. 5 (2009), pp. 515-520 (cit. on p. 10).

[60] M. Battiato, K. Carva, and P. M. Oppeneer. "Superdiffusive Spin Transport as a Mechanism of Ultrafast Demagnetization". Phys. Rev. Lett. 105 (2010), p. 027203 (cit. on pp. 10, 92).

[61] B. Koopmans, G. Malinowski, F. Dalla Longa, D. Steiauf, M. Fähnle, T. Roth, M. Cinchetti, and M. Aeschlimann. "Explaining the Paradoxical Diversity of Ultrafast Laser-Induced Demagnetization”. Nat. Mater. 9 (2010), pp. 259-265 (cit. on p. 10).

[62] M. De Graef. Introduction to Conventional Transmission Electron Microscopy. Cambridge: Cambridge University Press, 2003 (cit. on pp. 11-14, 17, 19, 30, 34, 62, 97).

[63] M. Reiser. Theory and Design of Charged Particle Beams. 2nd ed. Weinheim: WileyVCH Verlag GmbH \& Co., 2008 (cit. on pp. 11, 16, 23-25).

[64] D. B. Williams and C. B. Carter. Transmission Electron Microscopy: A Textbook for Materials Science. 2nd ed. Springer US, 2009 (cit. on p. 11).

[65] Y. Aharonov and D. Bohm. "Significance of Electromagnetic Potentials in the Quantum Theory”. Phys. Rev. 115 (1959), pp. 485-491 (cit. on pp. 11, 59). 
[66] M. De Graef and Y. Zhu. Magnetic Imaging and Its Applications to Materials. Academic Press, 2000 (cit. on pp. 11, 17, 34, 57).

[67] A. K. Petford-Long and M. De Graef. "Lorentz Microscopy". Characterization of Materials. John Wiley \& Sons, Inc., 2002 (cit. on pp. 11, 59).

[68] H. Lichte. "Electron Holography". Handbook of Nanoscopy. John Wiley \& Sons, Ltd, 2012, pp. 153-220 (cit. on pp. 12, 19).

[69] L. Reimer and H. Kohl. Transmission Electron Microscopy: Physics of Image Formation. 5th ed. New York: Springer-Verlag, 2008 (cit. on pp. 12, 17, 30, 34).

[70] H. Lichte and M. Lehmann. "Electron Holography — Basics and Applications". Rep. Prog. Phys. 71 (2008), p. 016102 (cit. on pp. 13-16, 22, 27).

[71] O. L. Krivanek. "Three-Fold Astigmatism in High-Resolution Transmission Electron Microscopy". Ultramicroscopy 55 (1994), pp. 419-433 (cit. on p. 14).

[72] R. E. Dunin-Borkowski, T. Kasama, M. Beleggia, and G. Pozzi. "Lorentz Microscopy and Electron Holography of Magnetic Materials". Handbook of Nanoscopy. John Wiley \& Sons, Ltd, 2012, pp. 221-251 (cit. on pp. 17, 19).

[73] A. Tonomura. "Applications of Electron Holography". Rev. Mod. Phys. 59 (1987), pp. 639-669 (cit. on pp. 19, 22, 27, 50).

[74] A. Tonomura, T. Matsuda, J. Endo, T. Arii, and K. Mihama. "Direct Observation of Fine Structure of Magnetic Domain Walls by Electron Holography". Phys. Rev. Lett. 44 (1980), pp. 1430-1433 (cit. on p. 19).

[75] A. Tonomura. "Electron Holography of Magnetic Materials and Observation of Flux-Line Dynamics". Ultramicroscopy 47 (1992), pp. 419-424 (cit. on p. 19).

[76] R. E. Dunin-Borkowski, M. R. McCartney, B. Kardynal, D. J. Smith, and M. R. Scheinfein. "Switching Asymmetries in Closely Coupled Magnetic Nanostructure Arrays". Appl. Phys. Lett. 75 (1999), pp. 2641-2643 (cit. on p. 19).

[77] A. Kovács, J. Caron, A. S. Savchenko, N. S. Kiselev, K. Shibata, Z.-A. Li, N. Kanazawa, Y. Tokura, S. Blügel, and R. E. Dunin-Borkowski. "Mapping the Magnetization Fine Structure of a Lattice of Bloch-Type Skyrmions in an FeGe Thin Film". Appl. Phys. Lett. 111 (2017), p. 192410 (cit. on p. 19).

[78] R. D. Gomez, T. V. Luu, A. O. Pak, K. J. Kirk, and J. N. Chapman. "Domain Configurations of Nanostructured Permalloy Elements". Journal of Applied Physics 85 (1999), pp. 6163-6165 (cit. on p. 19).

[79] S. McVitie, D. McGrouther, S. McFadzean, D. A. MacLaren, K. J. O'Shea, and M. J. Benitez. "Aberration Corrected Lorentz Scanning Transmission Electron Microscopy". Ultramicroscopy 152 (2015), pp. 57-62 (cit. on p. 19).

[80] D. Gabor and W. L. Bragg. "Microscopy by Reconstructed Wave-Fronts". Proceedings of the Royal Society of London. Series A. Mathematical and Physical Sciences 197 (1949), pp. 454-487 (cit. on p. 22).

[81] E. N. Leith and J. Upatnieks. "Reconstructed Wavefronts and Communication Theory*". J. Opt. Soc. Am., JOSA 52 (1962), pp. 1123-1130 (cit. on p. 22).

[82] G. Möllenstedt and H. Düker. "Beobachtungen und Messungen an Biprisma-Interferenzen mit Elektronenwellen". Z. Physik 145 (1956), pp. 377-397 (cit. on p. 22). 
[83] M. Lehmann. "Influence of the Elliptical Illumination on Acquisition and Correction of Coherent Aberrations in High-Resolution Electron Holography". Ultramicroscopy 100 (2004), pp. 9-23 (cit. on pp. 22, 23).

[84] P. Hawkes and E. Kasper. Principles of Electron Optics. London: Academic Press, 1988 (cit. on pp. 23-27, 34, 62, 66).

[85] M. Born and E. Wolf. Principles of Optics - Electromagnetic Theory of Propagation, Interference and Diffraction of Light. 6th ed. Kronberg: Pergamon Press, 1989 (cit. on pp. 23, 26, 27).

[86] L. Landau and E. Lifshitz. Course on Theoretical Physics - Mechanics. 3rd. Vol. 1. Oxford: Reed Educational and Professional Reading Ltd., 1981 (cit. on p. 24).

[87] C. A. Brau. "What Brightness Means". The Physics and Applications of High Brightness Electron Beams. WORLD SCIENTIFIC, 2003, pp. 20-27 (cit. on pp. 25, 26).

[88] M. Kuwahara, S. Kusunoki, X. G. Jin, T. Nakanishi, Y. Takeda, K. Saitoh, T. Ujihara, H. Asano, and N. Tanaka. "30-kV Spin-Polarized Transmission Electron Microscope with GaAs-GaAsP Strained Superlattice Photocathode". Appl. Phys. Lett. 101 (2012), p. 033102 (cit. on p. 28).

[89] K. Bücker, M. Picher, O. Crégut, T. LaGrange, B. W. Reed, S. T. Park, D. J. Masiel, and F. Banhart. "Electron Beam Dynamics in an Ultrafast Transmission Electron Microscope with Wehnelt Electrode". Ultramicroscopy 171 (2016), pp. 8-18 (cit. on p. 28).

[90] S. Ji, L. Piazza, G. Cao, S. T. Park, B. W. Reed, D. J. Masiel, and J. Weissenrieder. "Influence of Cathode Geometry on Electron Dynamics in an Ultrafast Electron Microscope". Structural Dynamics 4 (2017), p. 054303 (cit. on p. 28).

[91] S. V. Andreev, S. A. Aseev, V. N. Bagratashvili, N. S. Vorob'ev, A. A. Ishchenko, V. O. Kompanets, A. L. Malinovsky, B. N. Mironov, A. A. Timofeev, S. V. Chekalin, E. V. Shashkov, and E. A. Ryabov. "Ultrafast Transmission Electron Microscope for Studying the Dynamics of the Processes Induced by Femtosecond Laser Beams". Quantum Electron. 47 (2017), p. 116 (cit. on p. 28).

[92] H. Dömer and O. Bostanjoglo. "High-Speed Transmission Electron Microscope". Rev. Sci. Instrum. 74 (2003), pp. 4369-4372 (cit. on pp. 28, 29).

[93] T. LaGrange, M. R. Armstrong, K. Boyden, C. G. Brown, G. H. Campbell, J. D. Colvin, W. J. DeHope, A. M. Frank, D. J. Gibson, F. V. Hartemann, J. S. Kim, W. E. King, B. J. Pyke, B. W. Reed, M. D. Shirk, R. M. Shuttlesworth, B. C. Stuart, B. R. Torralva, and N. D. Browning. "Single-Shot Dynamic Transmission Electron Microscopy". Appl. Phys. Lett. 89 (2006), p. 044105 (cit. on p. 28).

[94] M. R. Armstrong, K. Boyden, N. D. Browning, G. H. Campbell, J. D. Colvin, W. J. DeHope, A. M. Frank, D. J. Gibson, F. Hartemann, J. S. Kim, W. E. King, T. B. LaGrange, B. J. Pyke, B. W. Reed, R. M. Shuttlesworth, B. C. Stuart, and B. R. Torralva. "Practical Considerations for High Spatial and Temporal Resolution Dynamic Transmission Electron Microscopy". Ultramicroscopy 107 (2007), pp. 356-367 (cit. on p. 28). 
[95] N. D. Browning, G. H. Campbell, J. E. Evans, T. B. LaGrange, K. L. Jungjohann, J. S. Kim, D. J. Masiel, and B. W. Reed. "Dynamic Transmission Electron Microscopy". Handbook of Nanoscopy. Weinheim: John Wiley \& Sons, Ltd, 2012, pp. 309-343 (cit. on pp. 28, 29).

[96] V. A. Lobastov, R. Srinivasan, and A. H. Zewail. "Four-Dimensional Ultrafast Electron Microscopy". PNAS 102 (2005), pp. 7069-7073 (cit. on pp. 28, 29).

[97] T. van Oudheusden, E. F. de Jong, S. B. van der Geer, W. P. E. M. O. 't Root, O. J. Luiten, and B. J. Siwick. "Electron Source Concept for Single-Shot Sub-100 \{fs\} Electron Diffraction in the 100 \{keV\} Range". Journal of Applied Physics 102 (2007), p. 093501 (cit. on p. 29).

[98] T. Matsuda, A. Fukuhara, T. Yoshida, S. Hasegawa, A. Tonomura, and Q. Ru. "Computer Reconstruction from Electron Holograms and Observation of Fluxon Dynamics". Phys. Rev. Lett. 66 (1991), pp. 457-460 (cit. on p. 29).

[99] T. Matsuda, K. Harada, H. Kasai, O. Kamimura, and A. Tonomura. "Observation of Dynamic Interaction of Vortices with Pinning Centers by Lorentz Microscopy". Science 271 (1996), pp. 1393-1395 (cit. on p. 29).

[100] I. S. Weir, J. N. Chapman, I. S. Molchanov, D. M. Titterington, and J. Rose. "Observation and Modelling of Magnetization Reversal in Multilayers Supporting Perpendicular Magnetization". J. Phys. D: Appl. Phys. 32 (1999), p. 395 (cit. on p. 29).

[101] M. Mochizuki, X. Z. Yu, S. Seki, N. Kanazawa, W. Koshibae, J. Zang, M. Mostovoy, Y. Tokura, and N. Nagaosa. "Thermally Driven Ratchet Motion of a Skyrmion Microcrystal and Topological Magnon Hall Effect". Nat. Mater. 13 (2014), pp. 241246 (cit. on pp. 29, 92).

[102] J. Rajeswari, P. Huang, G. F. Mancini, Y. Murooka, T. Latychevskaia, D. McGrouther, M. Cantoni, E. Baldini, J. S. White, A. Magrez, T. Giamarchi, H. M. Rønnow, and F. Carbone. "Filming the Formation and Fluctuation of Skyrmion Domains by CryoLorentz Transmission Electron Microscopy". PNAS 112 (2015), pp. 14212-14217 (cit. on pp. 29, 92).

[103] S. Pöllath, J. Wild, L. Heinen, T. N. G. Meier, M. Kronseder, L. Tutsch, A. Bauer, H. Berger, C. Pfleiderer, J. Zweck, A. Rosch, and C. H. Back. "Dynamical Defects in Rotating Magnetic Skyrmion Lattices". Phys. Rev. Lett. 118 (2017), p. 207205 (cit. on pp. 29, 92).

[104] G. Berruto, I. Madan, Y. Murooka, G. M. Vanacore, E. Pomarico, J. Rajeswari, R. Lamb, P. Huang, A. J. Kruchkov, Y. Togawa, T. LaGrange, D. McGrouther, H. M. Rønnow, and F. Carbone. "Laser-Induced Skyrmion Writing and Erasing in an Ultrafast Cryo-Lorentz Transmission Electron Microscope". Phys. Rev. Lett. 120 (2018), p. 117201 (cit. on pp. 29, 30).

[105] B. Barwick, D. J. Flannigan, and A. H. Zewail. "Photon-Induced Near-Field Electron Microscopy". Nature 462 (2009), pp. 902-906 (cit. on pp. 32, 49).

[106] H. Boersch. "Experimentelle Bestimmung der Energieverteilung in thermisch ausgelösten Elektronenstrahlen". Z. Physik 139 (1954), pp. 115-146 (cit. on p. 34).

[107] K. H. Loeffler. "Energy-Spread Generation in Electron-Optical Instruments". Z Angew Phys 27 (1969), p. 145 (cit. on p. 35). 
[108] N. Bach, T. Domröse, A. Feist, T. Rittmann, S. Strauch, C. Ropers, and S. Schäfer. "Coulomb Interactions in High-Coherence Femtosecond Electron Pulses from Tip Emitters". Structural Dynamics 6 (2019), p. 014301 (cit. on pp. 35, 45-48, 51, 83).

[109] N. Rubiano da Silva, M. Möller, A. Feist, H. Ulrichs, C. Ropers, and S. Schäfer. "Nanoscale Mapping of Ultrafast Magnetization Dynamics with Femtosecond Lorentz Microscopy". Phys. Rev. X 8 (2018), p. 031052 (cit. on pp. 37, 58, 75, 81).

[110] J. Raabe, R. Pulwey, R. Sattler, T. Schweinböck, J. Zweck, and D. Weiss. "Magnetization Pattern of Ferromagnetic Nanodisks". J. Appl. Phys. 88 (2000), pp. 4437-4439 (cit. on pp. 37, 38, 59).

[111] M. Schneider, H. Hoffmann, and J. Zweck. "Lorentz Microscopy of Circular Ferromagnetic Permalloy Nanodisks". Appl. Phys. Lett. 77 (2000), pp. 2909-2911 (cit. on pp. 37, 38).

[112] J. K. Ha, R. Hertel, and J. Kirschner. "Micromagnetic Study of Magnetic Configurations in Submicron Permalloy Disks". Phys. Rev. B 67 (2003), p. 224432 (cit. on p. 38).

[113] H. Y. Wong, J. N. Chapman, S. McVitie, and S. J. Hefferman. "The Influence of Evaporation Rate on the Domain Structures of Permalloy and Cobalt Small Magnetic Particles". Journal of Magnetism and Magnetic Materials 104-107 (1992), pp. 329330 (cit. on p. 38).

[114] S. Kasai, P. Fischer, M.-Y. Im, K. Yamada, Y. Nakatani, K. Kobayashi, H. Kohno, and T. Ono. "Probing the Spin Polarization of Current by Soft X-Ray Imaging of Current-Induced Magnetic Vortex Dynamics". Phys. Rev. Lett. 101 (2008), p. 237203 (cit. on p. 38).

[115] M.-Y. Im, P. Fischer, K. Yamada, T. Sato, S. Kasai, Y. Nakatani, and T. Ono. "Symmetry Breaking in the Formation of Magnetic Vortex States in a Permalloy Nanodisk". Nat. Commun. 3 (2012), p. 983 (cit. on p. 38).

[116] D. R. Lide. CRC Handbook of Chemistry and Physics. New York: CRC Press, 2002 (cit. on pp. 40, 80).

[117] C. Kittel. Introduction to Solid State Physics. 8th Edition. John Wiley \& Sons, 2004 (cit. on p. 41).

[118] R. J. Wakelin and E. L. Yates. "A Study of the Order-Disorder Transformation in Iron-Nickel Alloys in the Region FeNi 3". Proc. Phys. Soc. B 66 (1953), p. 221 (cit. on p. 41).

[119] T. Hibi and S. Takahashi. "Relation between Coherence of Electron Beam and Contrast of Electron Image". Z. angew. Physik 27 (1969), pp. 132-138 (cit. on p. 50).

[120] V. V. Volkov, Y. Zhu, and M. De Graef. "A New Symmetrized Solution for Phase Retrieval Using the Transport of Intensity Equation". Micron 33 (2002), pp. 411-416 (cit. on p. 57).

[121] A. Kohn and A. Habibi. "Adapting a JEM-2100F for Magnetic Imaging by Lorentz TEM”. JEOL News 47 (2012), pp. 17-22 (cit. on p. 62). 
[122] F. Thon. "Notizen: Zur Defokussierungsabhängigkeit Des Phasenkontrastes Bei Der Elektronenmikroskopischen Abbildung". Z. Für Naturforschung A 21 (1966), pp. 476-478 (cit. on p. 62).

[123] O. Krivanek. "Method for Determining the Coefficiente of Spherical Aberration from a Single Electron Micrograph". Optik 45 (1976), pp. 97-101 (cit. on p. 62).

[124] W. M. J. Coene and T. J. J. Denteneer. "Improved Methods for the Determination of the Spherical Aberration Coefficient in High-Resolution Electron Microscopy from Micrographs of an Amorphous Object". Ultramicroscopy 38 (1991), pp. 225-233 (cit. on p. 62).

[125] M. Beleggia, M. A. Schofield, Y. Zhu, M. Malac, Z. Liu, and M. Freeman. "Quantitative Study of Magnetic Field Distribution by Electron Holography and Micromagnetic Simulations". Appl. Phys. Lett. 83 (2003), pp. 1435-1437 (cit. on p. 65).

[126] E. Feldtkeller and H. Thomas. "Struktur und Energie von Blochlinien in dünnen ferromagnetischen Schichten”. Phys. kondens. Mater. 4 (1965), pp. 8-14 (cit. on p. 73).

[127] S. Mathias, C. La-O-Vorakiat, P. Grychtol, P. Granitzka, E. Turgut, J. M. Shaw, R. Adam, H. T. Nembach, M. E. Siemens, S. Eich, C. M. Schneider, T. J. Silva, M. Aeschlimann, M. M. Murnane, and H. C. Kapteyn. "Probing the Timescale of the Exchange Interaction in a Ferromagnetic Alloy". PNAS 109 (2012), pp. 4792-4797 (cit. on p. 80).

[128] J. Walowski, G. Müller, M. Djordjevic, M. Münzenberg, M. Kläui, C. A. F. Vaz, and J. A. C. Bland. "Energy Equilibration Processes of Electrons, Magnons, and Phonons at the Femtosecond Time Scale". Phys. Rev. Lett. 101 (2008), p. 237401 (cit. on p. 80).

[129] G. M. Vanacore, I. Madan, G. Berruto, K. Wang, E. Pomarico, R. J. Lamb, D. McGrouther, I. Kaminer, B. Barwick, F. J. G. de Abajo, and F. Carbone. "Attosecond Coherent Control of Free-Electron Wave Functions Using Semi-Infinite Light Fields". Nat. Commun. 9 (2018), p. 2694 (cit. on p. 87).

[130] S. Loth, M. Etzkorn, C. P. Lutz, D. M. Eigler, and A. J. Heinrich. "Measurement of Fast Electron Spin Relaxation Times with Atomic Resolution". Science 329 (2010), pp. 1628-1630 (cit. on p. 91).

[131] T. Taniuchi, Y. Kotani, and S. Shin. "Ultrahigh-Spatial-Resolution Chemical and Magnetic Imaging by Laser-Based Photoemission Electron Microscopy". Review of Scientific Instruments 86 (2015), p. 023701 (cit. on p. 91).

[132] O. Kfir, S. Zayko, C. Nolte, M. Sivis, M. Möller, B. Hebler, S. S. P. K. Arekapudi, D. Steil, S. Schäfer, M. Albrecht, O. Cohen, S. Mathias, and C. Ropers. "Nanoscale Magnetic Imaging Using Circularly Polarized High-Harmonic Radiation". Sci. Adv. 3 (2017), eaao4641 (cit. on p. 91).

[133] U. K. Rößler, A. N. Bogdanov, and C. Pfleiderer. "Spontaneous Skyrmion Ground States in Magnetic Metals". Nature 442 (2006), pp. 797-801 (cit. on p. 92).

[134] S. Mühlbauer, B. Binz, F. Jonietz, C. Pfleiderer, A. Rosch, A. Neubauer, R. Georgii, and P. Böni. "Skyrmion Lattice in a Chiral Magnet". Science 323 (2009), pp. 915-919 (cit. on p. 92). 
[135] C. Jin, Z.-A. Li, A. Kovács, J. Caron, F. Zheng, F. N. Rybakov, N. S. Kiselev, H. Du, S. Blügel, M. Tian, Y. Zhang, M. Farle, and R. E. Dunin-Borkowski. "Control of Morphology and Formation of Highly Geometrically Confined Magnetic Skyrmions". Nat. Commun. 8 (2017), p. 15569 (cit. on p. 92).

[136] S.-Z. Lin, A. Saxena, and C. D. Batista. "Skyrmion Fractionalization and Merons in Chiral Magnets with Easy-Plane Anisotropy". Phys. Rev. B 91 (2015), p. 224407 (cit. on p. 92).

[137] X. Z. Yu, W. Koshibae, Y. Tokunaga, K. Shibata, Y. Taguchi, N. Nagaosa, and Y. Tokura. "Transformation between Meron and Skyrmion Topological Spin Textures in a Chiral Magnet". Nature 564 (2018), p. 95 (cit. on p. 92).

[138] E. Feldtkeller. "Mikromagnetisch Stetige Und Unstetige Magnetisierungskonfigurationen". Z. angew. Physik 19 (1965), pp. 530-536 (cit. on p. 92).

[139] W. Döring. "Point Singularities in Micromagnetism". Journal of Applied Physics 39 (1968), pp. 1006-1007 (cit. on p. 92).

[140] A. Fert, V. Cros, and J. Sampaio. "Skyrmions on the Track". Nat. Nanotechnol. 8 (2013), pp. 152-156 (cit. on p. 92).

[141] R. Tomasello, E. Martinez, R. Zivieri, L. Torres, M. Carpentieri, and G. Finocchio. "A Strategy for the Design of Skyrmion Racetrack Memories". Sci. Rep. 4 (2014), p. 6784 (cit. on p. 92).

[142] Y. Tserkovnyak and J. Xiao. "Energy Storage via Topological Spin Textures". Phys. Rev. Lett. 121 (2018), p. 127701 (cit. on p. 92).

[143] W. Jiang, P. Upadhyaya, W. Zhang, G. Yu, M. B. Jungfleisch, F. Y. Fradin, J. E. Pearson, Y. Tserkovnyak, K. L. Wang, O. Heinonen, S. G. E. te Velthuis, and A. Hoffmann. "Blowing Magnetic Skyrmion Bubbles". Science 349 (2015), pp. 283286 (cit. on p. 92).

[144] S. Woo, K. Litzius, B. Krüger, M.-Y. Im, L. Caretta, K. Richter, M. Mann, A. Krone, R. M. Reeve, M. Weigand, P. Agrawal, I. Lemesh, M.-A. Mawass, P. Fischer, M. Kläui, and G. S. D. Beach. "Observation of Room-Temperature Magnetic Skyrmions and Their Current-Driven Dynamics in Ultrathin Metallic Ferromagnets". Nat Mater 15 (2016), pp. 501-506 (cit. on p. 92).

[145] K. Litzius, I. Lemesh, B. Krüger, P. Bassirian, L. Caretta, K. Richter, F. Büttner, K. Sato, O. A. Tretiakov, J. Förster, R. M. Reeve, M. Weigand, I. Bykova, H. Stoll, G. Schütz, G. S. D. Beach, and M. Kläui. "Skyrmion Hall Effect Revealed by Direct Time-Resolved X-Ray Microscopy". Nat. Phys. 13 (2017), pp. 170-175 (cit. on p. 92).

[146] S. A. Montoya, S. Couture, J. J. Chess, J. C. T. Lee, N. Kent, M.-Y. Im, S. D. Kevan, P. Fischer, B. J. McMorran, S. Roy, V. Lomakin, and E. E. Fullerton. "Resonant Properties of Dipole Skyrmions in Amorphous Fe/Gd Multilayers". Phys. Rev. B 95 (2017), p. 224405 (cit. on p. 92).

[147] M.-Y. Im, H.-S. Han, M.-S. Jung, Y.-S. Yu, S. Lee, S. Yoon, W. Chao, P. Fischer, J.-I. Hong, and K.-S. Lee. "Dynamics of the Bloch Point in an Asymmetric Permalloy Disk". Nat. Commun. 10 (2019), p. 593 (cit. on p. 92).

[148] S. S. P. Parkin, M. Hayashi, and L. Thomas. "Magnetic Domain-Wall Racetrack Memory”. Science 320 (2008), pp. 190-194 (cit. on p. 92). 
[149] K.-S. Ryu, L. Thomas, S.-H. Yang, and S. Parkin. "Chiral Spin Torque at Magnetic Domain Walls". Nat. Nanotechnol. 8 (2013), pp. 527-533 (cit. on p. 92).

[150] S. Emori, U. Bauer, S.-M. Ahn, E. Martinez, and G. S. D. Beach. "Current-Driven Dynamics of Chiral Ferromagnetic Domain Walls". Nat. Mater. 12 (2013), pp. 611616 (cit. on p. 92).

[151] Y. P. Ivanov, A. Chuvilin, S. Lopatin, and J. Kosel. "Modulated Magnetic Nanowires for Controlling Domain Wall Motion: Toward 3D Magnetic Memories". ACS Nano 10 (2016), pp. 5326-5332 (cit. on p. 92).

[152] A. Barman, S. Wang, J. Maas, A. R. Hawkins, S. Kwon, J. Bokor, A. Liddle, and H. Schmidt. "Size Dependent Damping in Picosecond Dynamics of Single Nanomagnets". Appl. Phys. Lett. 90 (2007), p. 202504 (cit. on p. 93).

[153] B. K. Mahato, B. Rana, D. Kumar, S. Barman, S. Sugimoto, Y. Otani, and A. Barman. "Tunable Spin Wave Dynamics in Two-Dimensional Ni80Fe20 Nanodot Lattices by Varying Dot Shape". Appl. Phys. Lett. 105 (2014), p. 012406 (cit. on p. 93).

[154] A.-C. Milazzo, G. Moldovan, J. Lanman, L. Jin, J. C. Bouwer, S. Klienfelder, S. T. Peltier, M. H. Ellisman, A. I. Kirkland, and N.-H. Xuong. "Characterization of a Direct Detection Device Imaging Camera for Transmission Electron Microscopy". Ultramicroscopy 110 (2010), pp. 741-744 (cit. on p. 93).

[155] G. McMullan, A. R. Faruqi, D. Clare, and R. Henderson. "Comparison of Optimal Performance at 300keV of Three Direct Electron Detectors for Use in Low Dose Electron Microscopy". Ultramicroscopy 147 (2014), pp. 156-163 (cit. on p. 93).

[156] G. Nahrwold, J. M. Scholtyssek, S. Motl-Ziegler, O. Albrecht, U. Merkt, and G. Meier. "Structural, Magnetic, and Transport Properties of Permalloy for Spintronic Experiments". Journal of Applied Physics 108 (2010), p. 013907 (cit. on p. 97).

[157] D. Ozkaya L, R. M. Langford, W. L. Chan, and A. K. Petford-Long. "Effect of Ga Implantation on the Magnetic Properties of Permalloy Thin Films". Journal of Applied Physics 91 (2002), pp. 9937-9942 (cit. on p. 97).

[158] D. S. G. Pollock. Handbook of Time Series Analysis, Signal Processing, and Dynamics. Cambridge: Academic Press, 1999 (cit. on p. 101).

[159] D. G. Voelz and M. C. Roggemann. "Digital Simulation of Scalar Optical Diffraction: Revisiting Chirp Function Sampling Criteria and Consequences". Appl. Opt., AO 48 (2009), pp. 6132-6142 (cit. on p. 102).

[160] K. Giewekemeyer. "A Study on New Approaches in Coherent X-Ray Microscopy of Biological Specimens". PhD Thesis. Göttingen University, 2011 (cit. on p. 102). 


\section{Publications and Conferences}

\section{Publications related to this work}

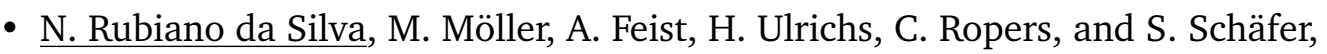
"Nanoscale Mapping of Ultrafast Magnetization Dynamics with Femtosecond Lorentz Microscopy", Physical Review X 8, 031052 (2018).

- A. Feist, N. Rubiano da Silva, W. Liang, C. Ropers, S. Schäfer, "Nanoscale diffractive probing of strain dynamics in ultrafast transmission electron microscopy", Structural Dynamics 5, 014302 (2018).

- A. Feist, N. Bach, N. Rubiano da Silva, T. Danz, M. Möller, K. E. Priebe, T. Domröse, J. G. Gatzmann, S. Rost, J. Schauss, S. Strauch, R. Bormann, M. Sivis, S. Schäfer, and C. Ropers, "Ultrafast transmission electron microscopy using a laser-driven field emitter: femtosecond resolution with a high coherence electron beam", Ultramicroscopy 176, 63-73 (2017).

- T. Eggebrecht, M. Möller, J. G. Gatzmann, N. Rubiano da Silva, A. Feist, U. Martens, H. Ulrichs, M. Münzenberg, C. Ropers, and S. Schäfer, "Light-Induced Metastable Magnetic Texture Uncovered by in situ Lorentz Microscopy", Physical Review Letters 118, 097203 (2017).

\section{Parts of this work have been presented on the following conferences}

- Ultrafast demagnetization dynamics imaged by time-resolved Lorentz microscopy, Ultrafast Magnetism Conference, 2017, Kaiserslautern 
- Ultrafast Lorentz microscopy of nanoscale magnetization dynamics, DPG Frühjahrstagung 2017, Dresden

- A light induced metastable magnetic texture uncovered by in-situ Lorentz microscopy, CMD26, 2016, Groningen, The Netherlands

- Imaging optically induced magnetization dynamics by Lorentz microscopy, Microscopy Conference, 2015, Göttingen 


\section{Acknowledgements}

Firstly, I want to express my gratitude to my main advisor, Claus Ropers, for giving me the opportunity to be part of such a great research group. Thank you for the guidance, the support for sharing my work in conferences and publications, and for the useful discussions.

I want to thank likewise Sascha Schäfer, for the advice and cooperation in great part of my $\mathrm{PhD}$ journey. Thank you for being available for my questions and for helping me, both for paper-writing and for dealing with simulation/microscope issues.

In addition, I thank Stefan Mathias, who acted both as a member of the advisory committee and as a second reviewer of this thesis. Thank you for the interest in my work and for your punctual remarks.

For providing me with financial support for a $\mathrm{PhD}$ in Germany, I want to acknowledge the funding agency in my home country, Brazil. The present work was realized with support of CNPq (National Council for Scientific and Technological Development), within the Science Without Borders Program (Dilma Rousseff administration). For further support on conference travels and research expenses, I acknowledge the German Collaborative Research Center SFB 1073.

I appreciate the collaboration with Henning Ulrichs, and I thank him for performing micromagnetic simulations of the sample system and for useful discussions.

My sincere thanks is also owed to those who carefully proofread parts of this thesis: Nora Bach, Armin Feist, Tyler Harvey, Murat Sivis, Gero Storeck, Simon Vogelgesang and Bart Wit. A further thanks to the UTEM team, for the cooperation and for making the lab environment somehow work for all of us. I include here also our great helper, Karin Ahlborn, for her friendly, prompt response and technical support.

I am very glad to thank also my whole working group, full of talented and friendly people. Not only for sharing lab snacks, optical components and Matlab scripts, but also for all the funny moments out of the working hours. 
Last but not least, I want to acknowledge all the support and love I have always got from my friends and my family. My special thanks to my parents and siblings, who always had my back since I was very young. And my warmest thanks to my husband Tobias and my daughter Stella, for all the patience and encouragement along the way, for the backstage assistance and for the joy of making the most of our time together. 